Provided for non-commercial research and education use. Not for reproduction, distribution or commercial use.

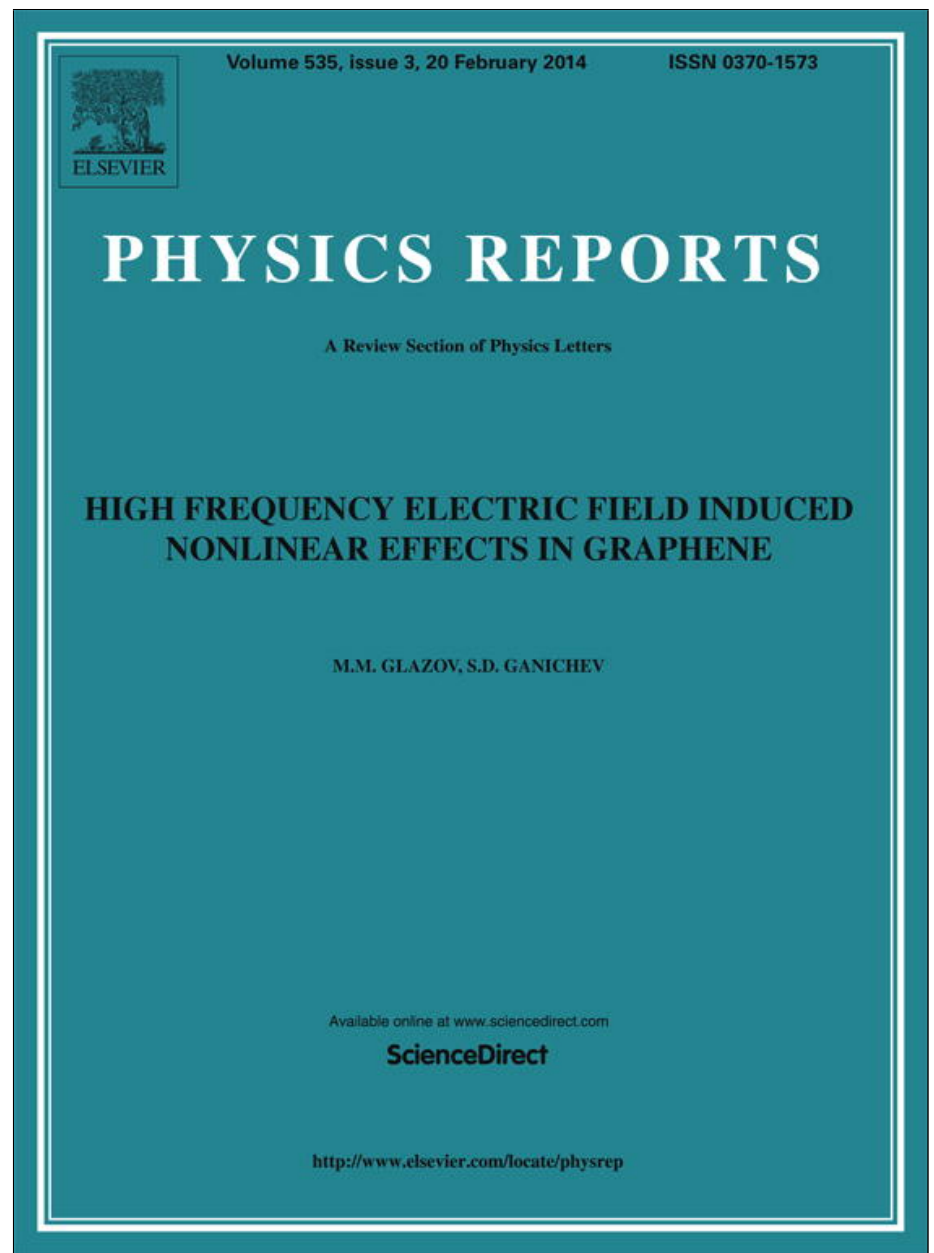

This article appeared in a journal published by Elsevier. The attached copy is furnished to the author for internal non-commercial research and education use, including for instruction at the authors institution and sharing with colleagues.

Other uses, including reproduction and distribution, or selling or licensing copies, or posting to personal, institutional or third party websites are prohibited.

In most cases authors are permitted to post their version of the article (e.g. in Word or Tex form) to their personal website or institutional repository. Authors requiring further information regarding Elsevier's archiving and manuscript policies are encouraged to visit:

http://www.elsevier.com/authorsrights 


\title{
High frequency electric field induced nonlinear effects in graphene
}

\author{
M.M. Glazov ${ }^{\mathrm{a}, *}$, S.D. Ganichev ${ }^{\mathrm{b}}$ \\ a Ioffe Physical-Technical Institute of the RAS, 194021 St.-Petersburg, Russia \\ ${ }^{\mathrm{b}}$ Terahertz Center, University of Regensburg, 93040, Regensburg, Germany
}

\section{A R T I C L E I N F O}

\section{Article history:}

Accepted 14 October 2013

Available online 19 October 2013

editor: G. E. W. Bauer

\section{Keywords:}

Graphene

Nonlinear high frequency transport

Nonlinear optics

Photocurrents

Ratchets

\begin{abstract}
A B S T R A C T
The nonlinear optical and optoelectronic properties of graphene with the emphasis on the processes of harmonic generation, frequency mixing, photon drag and photogalvanic effects as well as generation of photocurrents due to coherent interference effects, are reviewed. The article presents the state-of-the-art of this subject, including both recent advances and well-established results. Various physical mechanisms controlling transport are described in depth including phenomenological description based on symmetry arguments, models visualizing physics of nonlinear responses, and microscopic theory of individual effects.
\end{abstract}

(C) 2013 Elsevier B.V. All rights reserved.

\section{Contents}

1. Introduction.

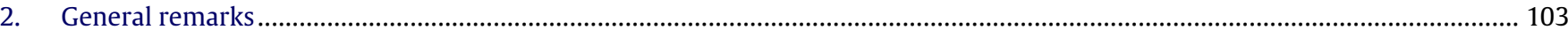

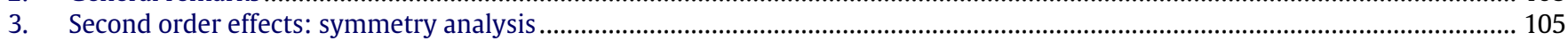

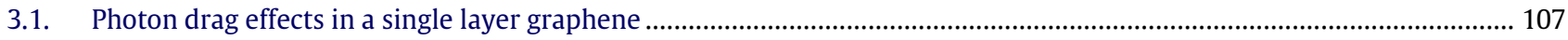

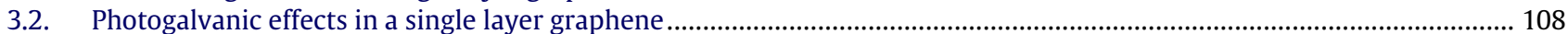

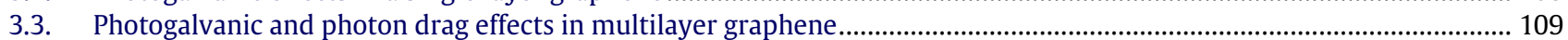

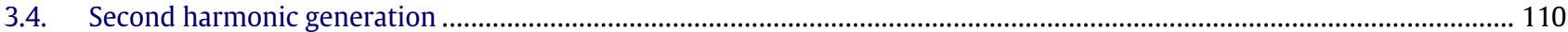

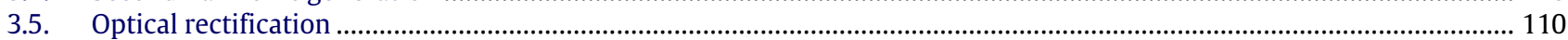

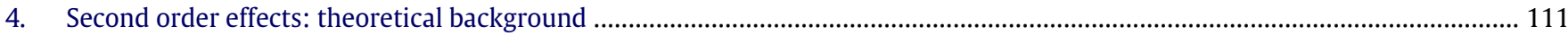

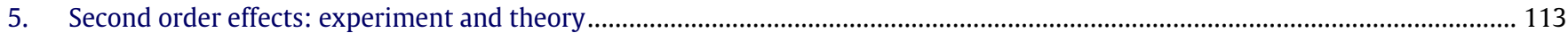

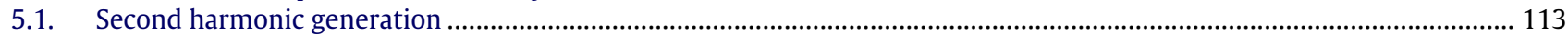

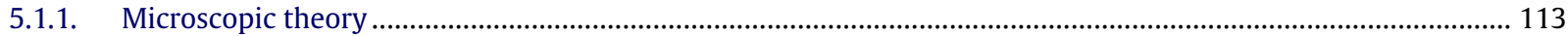

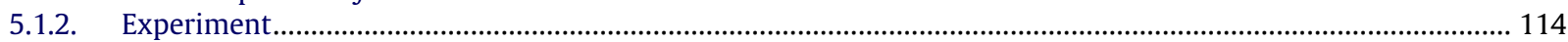

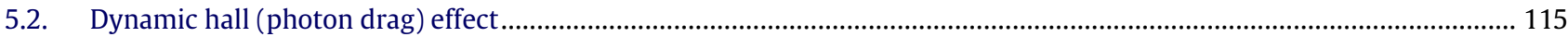

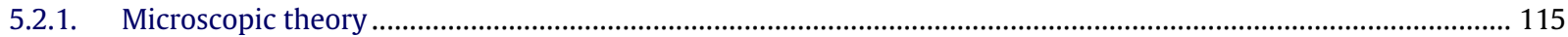

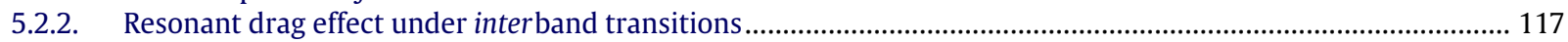

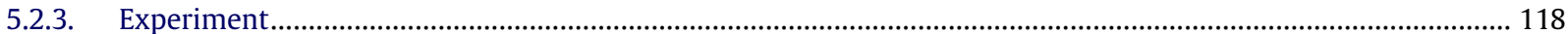

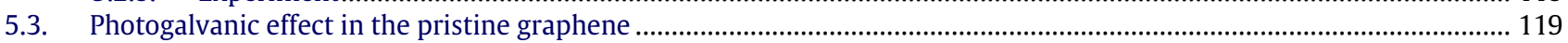

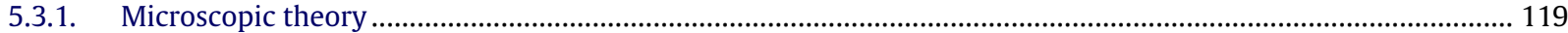

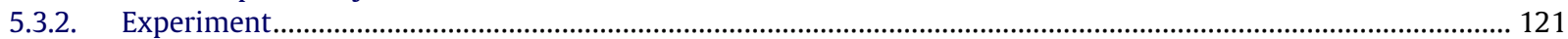

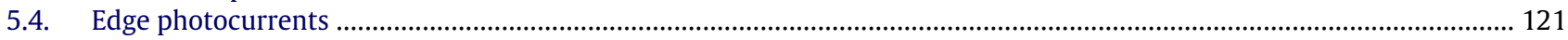

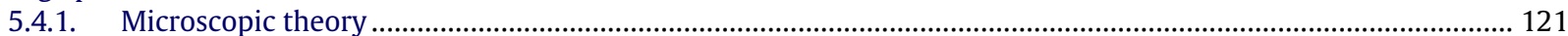

\footnotetext{
* Corresponding author. Tel.: +7 9119130436 .

E-mail addresses: glazov@coherent.ioffe.ru (M.M. Glazov), sergey.ganichev@physik.uni-regensburg.de (S.D. Ganichev).
} 


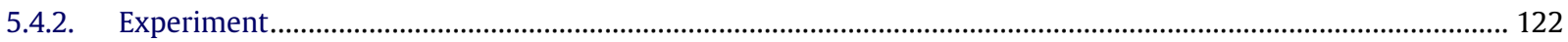

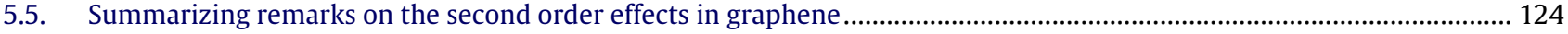

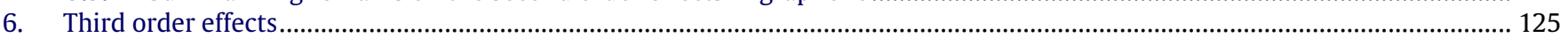

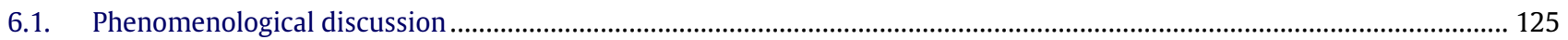

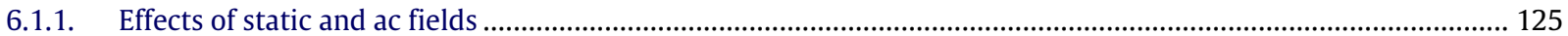

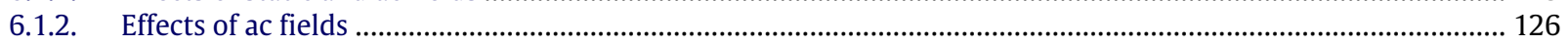

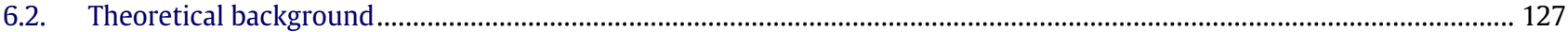

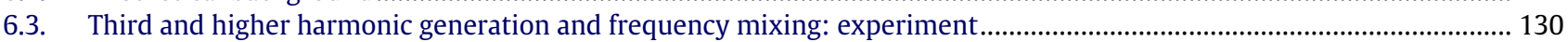

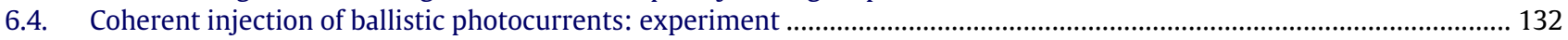

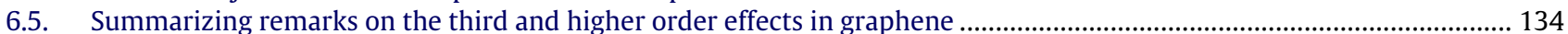

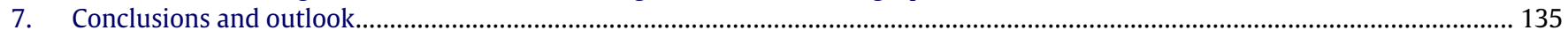

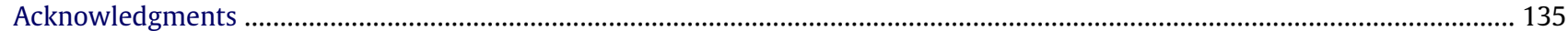

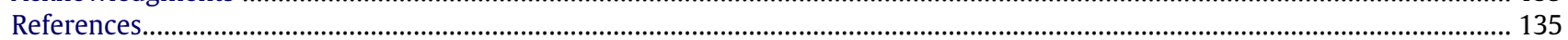

\section{Introduction}

The discovery of graphene opened a new era in material science. Graphene is the first truly two-dimensional (2D) crystal consisting of just a single layer of carbon atoms arranged in a hexagonal lattice [1-5]. The main consequence of such a crystal structure is the linear energy spectrum of electrons and holes, $\varepsilon_{\boldsymbol{p}}= \pm v|\boldsymbol{p}|$, where $v \approx c / 300$ is the effective speed, $c$ is the speed of light, $\boldsymbol{p}$ is the charge carrier momentum and signs \pm refer to the conduction and valence bands, which merge at $\boldsymbol{p}=0$ point, at the edges of the Brillouin zone [6-8]. Owing to a specific energy dispersion, graphene has revealed fascinating effects in a number of experiments. In particular, the linear coupling of the charge carriers energy with their momentum leads to a peculiar modification of the quantum Hall effect [2,9] and plays an important role in phase-coherent phenomena such as, e.g., weak localization [10,11], minimal electrical conductivity [2,3,12-14], Klein tunneling [15,16], etc., for reviews see $[4,5,17]$. The fact that the band structure resembles the dispersion relation of a massless relativistic particle has created enormous excitement since graphene provides an excellent model system for benchtop studies of quantum-electrodynamic effects $[18,19]$ making relativistic experiments in a solid state environment feasible [4,20,21]. Another important issue of this material is the presence of two valleys, each containing a Dirac cone. This constitutes a two-state degree of freedom, which was suggested to be used in valleytronics [22]. These and other specific features manifest themselves in a linear in electric field transport in graphene and have made it attractive for fundamental research and numerous applications, for review see, e.g. Refs. [23-26].

While linear in electric field phenomena in graphene are in focus of the current research, nonlinear transport effects, where the response is proportional to the higher powers of the electric field, are much less studied. In general, the such effects are caused by the redistribution of the charge carriers in the momentum and energy space induced by the radiation incident on the sample. The resulting nonequilibrium distribution can contain oscillating in time and space components as well as steady-state and spatially homogeneous ones. Hence, the radiation may cause both $a c$ and $d c$ current flows in a media, whose magnitudes are nonlinear functions of the field amplitude and whose components are sensitive to the radiation polarization. In conventional three- and two-dimensional semiconductors with parabolic energy dispersion, as well as in metals and dielectric crystals, a large number of nonlinear effects was observed and studied in great details. Harmonic generation, frequency mixing, optical rectification, linear and circular photogalvanic effects, photon drag effect, photoconductivity, coherently controlled ballistic charge currents, etc. are the subjects of intense research and already found a number of applications [27-33]. Moreover, these effects have been proven to be a very efficient tool to study nonequilibrium optical and electronic processes in semiconductors and provide information about their fundamental properties. For instance, they provide an access to the symmetry, peculiarities of the band structure, processes of electron momentum, energy/spin relaxation etc., as well as allow one to explore the processes of interaction of light with charge carriers (for review see, e.g. [27-35]). Concerning the carbon based systems, so far the nonlinear transport has been extensively studied for carbon nanotubes and carbon films [36-43], for review see, e.g. [44].

Naturally, nonlinear effects have attracted attention in graphene [45,46], where a number of phenomena, including second [47-51] and third [48,52-54] harmonic generation, frequency mixing [48,52,55-57], photon drag effects [58-60], chiral edge photocurrents [61], "bulk" photogalvanic effects [62], coherent current injection [63-65], time-resolved photocurrents [66,67], photocurrents in graphene pn-junctions [68-70], spatial self-phase modulation [71] and optical Kerr effect $[72,73],{ }^{1}$ have been already addressed theoretically and experimentally. These works demonstrated that the microscopic mechanisms of such effects in graphene can be quite different from their counterparts in ordinary semiconductor systems. Moreover, all the effects observed in graphene have a common feature: they are strongly enhanced compared with their analogues in semiconductors. The reasons for this, on the first glance surprising, fact are the high electron velocity and the linear dispersion in graphene. Indeed, the large velocity of electrons in graphene, as compared with typical semiconductor systems, obviously implies the efficient radiation-electron motion coupling. As for the electron dispersion,

\footnotetext{
1 Spatial self-phase modulation detected in colloidal dispersion of graphene sheets in organic solvents as well as optical Kerr effect in this system are out of scope of the present review.
} 
it crucially affects the details of optical transitions in the electron momentum space (k-space). In particular, the gapless, linear dispersion allows one to easily suppress some of the optical excitation channels, e.g., leading to the resonant nonlinear response [60]. Moreover, the nonlinearities and the nonlinear response can be enhanced via the excitation of the plasmonic waves in graphene [74-76]. Therefore, although being limited to a rather small amount of theoretical and even less experimental works, current research has already demonstrated that studying of nonlinear transport provides an access to various properties of graphene. Among others, these studies have proved that graphene, as a nonlinear element, is a promising material for a variety of different applications and may be used for the development of novel electronic devices for microwave-, terahertz- and optoelectronic applications. Thus, the experimental and theoretical research in the field of nonlinear graphene optics and optoelectronics becomes an important task.

The paper is aimed to give an overview of the key properties of graphene as a nonlinear material, to outline the main theoretical and experimental results obtained in the nonlinear physics of graphene so far, and stimulate further studies of these effects in this material. We start with the brief introduction to the nonlinear phenomena in graphene. Then, we describe the second and third order effects. Each class of the effects is presented in a similar way: we start with the phenomenological analysis of different phenomena based on the symmetry arguments, provide theoretical background and, one by one, give an overview of the microscopic theory and the main experimental results. Finally, we summarize the results and discuss prospectives of future theoretical and experimental studies of the nonlinear electromagnetic response of graphene.

\section{General remarks}

The standard way to treat the nonlinear effects without going into microscopic details makes use of the symmetry arguments. This approach allows one to conclude on the experimental geometry and conditions of observation of the effect under consideration as well as to describe its variation with change of macroscopic parameters, such as intensity of the radiation, its polarization and angle of incidence without knowing of the microscopic origin. In this way, the electron ensemble response to the external field can be most conveniently characterized by the coordinate- and time-dependent electric current density $\boldsymbol{j}(\boldsymbol{r}, t)$. It is expanded in the power series in the external alternating electric field $\boldsymbol{E}(\omega, \boldsymbol{q})$ taken in the form of a plane wave

$$
\boldsymbol{E}(\boldsymbol{r}, t)=\boldsymbol{E}(\omega, \boldsymbol{q}) \mathrm{e}^{-\mathrm{i} \omega t+\mathrm{i} \boldsymbol{q r}}+\boldsymbol{E}^{*}(\omega, \boldsymbol{q}) \mathrm{e}^{\mathrm{i} \omega t-\mathrm{i} \boldsymbol{r} \boldsymbol{r}},
$$

where $\omega$ is the radiation frequency and $\boldsymbol{q}$ is its wavevector. By that it has a form

$$
\begin{aligned}
j_{\alpha}(\boldsymbol{r}, t)= & {\left[\sigma_{\alpha \beta}^{(1)} E_{\beta}(\omega, \boldsymbol{q}) \mathrm{e}^{-\mathrm{i} \omega t+\mathrm{i} \boldsymbol{q r}}+\text { c.c. }\right]+\left[\sigma_{\alpha \beta \gamma}^{\left(2^{\prime}\right)} E_{\beta}(\omega, \boldsymbol{q}) E_{\gamma}(\omega, \boldsymbol{q}) \mathrm{e}^{-2 \mathrm{i} \omega t+2 \mathrm{i} \boldsymbol{q} r}+\text { c.c. }\right] } \\
& +\sigma_{\alpha \beta \gamma}^{(2)} E_{\beta}(\omega, \boldsymbol{q}) E_{\gamma}^{*}(\omega, \boldsymbol{q})+\ldots
\end{aligned}
$$

Here Greek subscripts refer to the Cartesian coordinates, c.c. stands for the complex conjugate, and Eq. (2) is limited to the second order effects. While the first term in Eq. (2) describes the linear transport, the other terms are the second order in electric field and include: (i) the contribution oscillating as $\exp (-2 \mathrm{i} \omega t)$ responsible for the second harmonic generation (second term) and (ii) time-independent contribution yielding the directed (dc) current generation (last term). These nonlinear processes are characterized by the nonlinear conductivities $\sigma_{\alpha \beta \gamma}^{\left(2^{\prime}\right)}$ and $\sigma_{\alpha \beta \gamma}^{(2)}$, respectively, whose specific form will be detailed below in Section 3. The class of these phenomena can be extended by considering the nonlinear polarization $\boldsymbol{P}$, which is described by the equation similar to Eq. (2) and leads to, e.g. the optical rectification effect. The higher order effects in Eq. (2) like third harmonic generation are denoted by triple dot mark. The corresponding expressions and their description will be given in Section 6 .

On a very general level, the enhanced nonlinear properties of graphene can be illustrated by considering the classical motion of the charge carrier under the action of the harmonic electric field $\boldsymbol{E}(t)=\boldsymbol{E}_{0} \cos \omega t$, where $\boldsymbol{E}_{0}$ is the amplitude of the field, $\omega$ is its frequency and taking into account the linear energy dispersion, $\varepsilon_{\boldsymbol{p}}= \pm v|\boldsymbol{p}|$. The electron motion is described by the second Newton law

$$
\frac{d \boldsymbol{p}}{d t}=e \boldsymbol{E}_{0} \cos \omega t,
$$

where $e=-|e|$ is the electron charge. It follows from this equation that electron momentum exhibits harmonic oscillations $\boldsymbol{p}(t)=\boldsymbol{p}_{0}+\left(e \boldsymbol{E}_{0} / \omega\right) \sin \omega t$, where $\boldsymbol{p}_{0}$ is the initial value of the electron momentum. In contrast to usual semiconductor systems with parabolic or slightly nonparabolic dispersion, here the electron velocity, and, hence, other observable quantities like, e.g. electric current, dipole moment or emitted radiation, demonstrate strongly anharmonic temporal behavior. Indeed, taking into account that for a massless particle the absolute value of the velocity is fixed, and its direction is determined by the direction of the momentum, we have $[47,77]$

$$
\boldsymbol{v}(t)= \pm v \frac{\boldsymbol{p}}{|\boldsymbol{p}|}= \pm v \frac{e \boldsymbol{E}_{0}}{\left|e \boldsymbol{E}_{0}\right|} \operatorname{sign}[\sin \omega t]= \pm v \frac{e \boldsymbol{E}_{0}}{\left|e \boldsymbol{E}_{0}\right|} \frac{4}{\pi}\left(\sin \omega t+\frac{1}{3} \sin 3 \omega t+\frac{1}{5} \sin 5 \omega t+\cdots\right) .
$$

Here we assume that $p_{0} \ll e E_{0} / \omega$, signs + and - correspond to the electron in the conduction and valence band, respectively. In this simplified model, the nonlinear effects become important even at very small fields: The coefficient 
$\mathrm{a}$

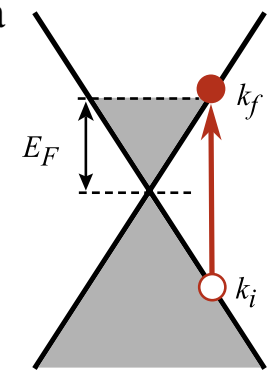

$\hbar \omega \geq 2 E_{F}$ b

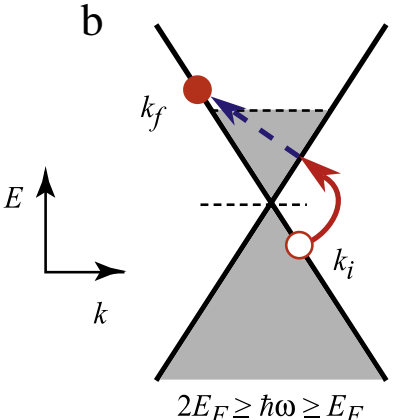

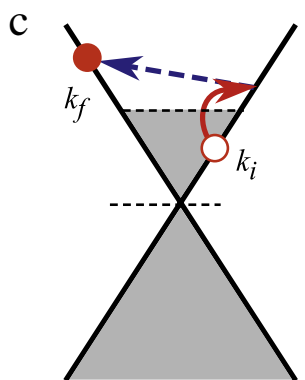

$E_{F} \geq \hbar \omega$

Fig. 1. Schematic illustration of the possible optical transitions: (a) direct interband transition, (b) indirect interband transition, (c) indirect intraband transition. Solid and dashed arrows show electron-photon interaction (solid arrows) and electron scattering caused by impurities or phonons (dashed arrows). Initial and final states of a photoexcited carrier with wavevectors $k_{i}$ and $k_{f}$ are shown by open and solid circles, respectively. Inequalities define the corresponding photon energy ranges.

at the third harmonic in the velocity is just $1 / 3$ of the first harmonic coefficient. For doped graphene with the typical Fermi energy $E_{F} \sim 100 \mathrm{meV}$ the estimations yield that the nonlinear response can already be observed at fields as low as $10^{2}-10^{3} \mathrm{~V} / \mathrm{cm}$ [74], being several orders of magnitude smaller than required for the same phenomena in other media.

Discussing various routes of nonlinearities in graphene, one should consider the relation between photon and Fermi energies, which governs the nonlinear response of any material via microscopic mechanism of light-matter coupling. Thus, before going in details of specific mechanism we address different regimes of optical excitation in graphene. The general description of radiation induced effects is based on the standard approach replacing the electron momentum $\boldsymbol{p}=\left(p_{x}, p_{y}\right)$ by $\boldsymbol{p}-e \boldsymbol{A} / c$ in Dirac Hamiltonian

$$
\hat{\mathscr{H}}(\boldsymbol{p})=v(\hat{\boldsymbol{\sigma}} \cdot \boldsymbol{p}),
$$

with $\boldsymbol{A}$ being vector potential of the electromagnetic field, ${ }^{2}$ and $\hat{\boldsymbol{\sigma}}=\left(\hat{\sigma}_{x}, \hat{\sigma}_{y}\right)$ is the vector composed of the Pauli matrices, which serve as basis matrices in the space of the electron states in the conduction and valence bands in the vicinity of Dirac point. $^{3}$ The change of frequency, and/or the Fermi energy, $E_{F}$, not only strongly influence the magnitude of the nonlinear phenomena, but may change the microscopic picture of their formation or, at certain conditions, may give rise to resonance responses. Several regimes of light-matter interaction depending on the photon energy, $\hbar \omega$, electron Fermi energy (in certain cases temperature) and its momentum relaxation rate, $1 / \tau$, are of importance. As a rule, physical problems of nonlinear transport are studied for graphene systems, for which the condition

$$
E_{F} \tau / \hbar \gg 1
$$

is fulfilled, allowing to consider electrons (holes) as free carriers. Moreover, taking into account that the energy distance from the Dirac point, $\varepsilon_{\boldsymbol{p}}=0$, to other bands in graphene is extremely large, exceeding $10 \mathrm{eV}[78,79]$, one can also disregard direct optical transitions involving other bands.

It follows then, that three regimes of radiation absorption are relevant to the discussed problem, namely, (i) direct interband transitions, (ii) indirect phonon or impurity assisted interband transitions and (iii) free-carrier absorption (Drudelike) due to indirect intraband transitions. These three processes are schematically shown in Fig. 1(a), (b), (c), respectively. To obtain direct transitions, obviously, the condition

$$
\hbar \omega \geqslant 2 E_{F},
$$

must be fulfilled. The indirect interband transitions become essential at

$$
E_{F} \leqslant \hbar \omega \leqslant 2 E_{F} .
$$

Finally, free carrier absorption caused by indirect intraband transitions may contribute for any relation between $\hbar \omega$ and $E_{F}$. Similar to the case of conventional semiconductors its role increases for larger wavelengths, and for

$$
\hbar \omega<E_{F}
$$

intraband transitions become dominant absorption processes. ${ }^{4}$

\footnotetext{
2 Hereafter we use the gauge where the scalar potential of electromagnetic field is absent, $\varphi=0$.

3 We note, that such an approximation is suitable only for electrons in a given valley of graphene, i.e. where the interaction with other (distant) bands is neglected. Moreover we disregarded the trigonal warping of the energy spectrum in each valley, which is pronounced for the energy distance from the Dirac point higher than $\sim 3 \mathrm{eV}$.

${ }^{4}$ Note, that the impurity ionization, multiphoton and lattice absorption, while being possible, are out of the scope of the present review.
} 
Besides the microscopic origin of the radiation absorption we distinguish the classical and quantum regimes of light-matter interaction, which are conventionally given by the relation between $\hbar \omega$ and $E_{F}$. In the classical regime characterized by

$$
\hbar \omega \ll E_{F},
$$

the electron dynamics can be described by means of Newton equations of motion and Boltzmann equation for the distribution function $f(\boldsymbol{p}, \boldsymbol{r}, t)$, which depends on electron momentum, $\boldsymbol{p}$, position, $\boldsymbol{r}$, and time, $t$. Such a description is valid for the arbitrary values of $\omega \tau$. We note also, that according to Eq. (4) if $\omega \tau \ll 1$, then the condition $\hbar \omega \ll E_{F}$ is automatically fulfilled. For photon energy approaching the Fermi energy or for even higher photon energies, at which Eq. (5d) does not hold, the quantum-mechanical treatment of the radiation interaction with electron system in graphene is required. In the intermediate frequency range, where $\hbar / \tau \ll \hbar \omega \ll E_{F}$, both classical and quantum-mechanical approaches merge.

All described regimes can easily be realized by variation of the photon energy and electron density in graphene. In the experiments reported so far, making use of the excitation with microwave/terahertz radiation and mid-infrared/visible light clearly corresponds to the classical or quantum mechanical regimes of the light-matter interaction, respectively. This is due to the fact that the nonlinear transport has been studied in ungated $n$ - and $p$-type graphene samples, which have rather high carrier density of the order of several times of $10^{12} \mathrm{~cm}^{-2}$ and, correspondingly, high Fermi energy ranging from 100 to $400 \mathrm{meV}$. Besides high Fermi energy, in all studied samples (exfoliated layers on $\mathrm{SiO}_{2} / \mathrm{Si}$ substrate, epitaxial graphene on $\mathrm{SiC}$ or CVD graphene) the electron mobility is quite low, about several thousands $\mathrm{cm}^{2} /$ Vs at room temperature. Such rather short scattering times $\tau$ ranging from units of $10^{-14}-10^{-12} \mathrm{~s}$ enable the investigation of nonlinear effects for the parameter $\omega \tau$ about unity giving rise to a number of specific effects for $\mathrm{THz} /$ microwave frequencies. In particular, processes sensitive to the radiation helicity become important and may dominate the nonlinear response for $\omega \tau \sim 1$, see Section 4 .

Before we begin the discussion of particular phenomena, we address one more important issue being crucial for all the nonlinear effect under study, namely, the variation of the radiation polarization state including degrees of linear and circular polarization. The controllable modification of the radiation polarization not only helps in the analysis of the mechanisms of the nonlinear response but also gives rise to new phenomena caused by transfer of the radiation angular momenta to the carriers in graphene. Below we show that the various contributions to the nonlinear response are proportional to the Stokes parameters. Therefore in almost all experiments aimed to nonlinear high frequency effects in graphene the polarization state of the radiation is controllably modified by means of standard dichroic elements like, e.g., $\lambda / 2$ and $\lambda / 4$ plates or Fresnel rhombus. By that, assuming the radiation propagates along positive $z$ axis, the Stokes parameters $[80,81]$ are given by

$$
\begin{aligned}
& S_{1}=\frac{\left|E_{x}\right|^{2}-\left|E_{y}\right|^{2}}{\left|E_{x}\right|^{2}+\left|E_{y}\right|^{2}}, \quad S_{2}=\frac{E_{x} E_{y}^{*}+E_{x}^{*} E_{y}}{\left|E_{x}\right|^{2}+\left|E_{y}\right|^{2}}, \\
& S_{3} \equiv P_{\text {circ }}=\mathrm{i} \frac{E_{x} E_{y}^{*}-E_{x}^{*} E_{y}}{\left|E_{x}\right|^{2}+\left|E_{y}\right|^{2}} .
\end{aligned}
$$

Note that as it will be shown below these parameters determine the nonlinear response to linear $\left(S_{1}, S_{2}\right)$ and circular $\left(S_{3}\right)$ light polarization. Particularly, $S_{1}$ and $S_{2}$ define the linear polarization of radiation in the $(x y)$ and rotated by $45^{\circ}$ coordinate frames, and $S_{3}$ describes the degree of circular polarization or helicity of radiation. Rotation of the polarizer in respect to the polarization plane of the linearly polarized laser radiation with $\boldsymbol{E}_{l} \| x$ results in the variation of the $S_{1}, S_{2}$ and $S_{3}$. In particular, rotation of the half-wave plate results in the linearly polarized radiation with

$$
S_{1} \propto \cos 2 \alpha, S_{2} \propto \sin 2 \alpha, S_{3}=0,
$$

where $\alpha=2 \beta$ defines the orientation of the polarization plane and $\beta$ is the angle between $\boldsymbol{E}_{l}$ the optical axis $c$. The radiation ellipticity, particularly, light helicity are conveniently varied by rotation of a quarter-wave plate by angle $\varphi$, resulting in

$$
S_{1} \propto \cos ^{2} 2 \varphi, S_{2} \propto \sin 4 \varphi, S_{3} \propto \sin 2 \varphi .
$$

We note that at oblique incidence, crucially needed for some nonlinear effects in graphene, the functional behavior of nonlinear contributions in Eq. (2) is also described by trigonometrical equations (7). This is in spite of the fact, that, strictly speaking, they are not directly given by the Stokes parameters $S_{1}, S_{2}, S_{3}$ in form of Eqs. (6).

\section{Second order effects: symmetry analysis}

The class of the second order effects includes second harmonic generation, $d c$ photocurrent generation, and optical rectification effect. Obviously, the magnitude of the second-order in the electric field response is linear in the radiation intensity $I=c|\boldsymbol{E}(\boldsymbol{q}, \omega)|^{2} / 2 \pi .^{5}$ The appearance and particular behavior of the effects upon variation of incidence angle and polarization state of the radiation are determined by the symmetry of the system. This is due to the fact that at a spatial inversion

\footnotetext{
5 We emphasize that the magnitude of electric field acting on the charge carriers in graphene differs from that of an incident wave owing to the presence of substrate, finite conductivity of graphene itself and interactions. These effects require additional analysis and disregarded hereinafter.
} 
the vector of electric current $\boldsymbol{j}$ changes its sign while quadratic combinations $E_{\alpha} E_{\beta}, E_{\alpha} E_{\beta}^{*}$ in Eq. (2) do not. Hence, the second order response is allowed if either (i) the spatial inversion is incompatible with the symmetry of the structure under study, or (ii) second-order conductivities $\sigma_{\alpha \beta \gamma}^{(2)}$ and $\sigma_{\alpha \beta \gamma}^{\left(2^{\prime}\right)}$ change their signs at spatial inversion. The latter is fulfilled, if components $\sigma_{\alpha \beta \gamma}$ are proportional to the components of the radiation wavevector $\mathbf{q}$. This is because both photon wavevector $\mathbf{q}$ and electric current $\boldsymbol{j}$ change their signs at the spatial inversion and symmetry allows the linear coupling between the current and photon wavevector in the second-order effects, $\boldsymbol{j} \propto \mathbf{q}|E|^{2}$. The sensitivity of the second-order phenomena to the spatial inversion reveals that peculiarities of the graphene structures, such as coupling with the substrate, presence of adatoms, terraces, ripples, edges, etc. become crucial. A further consequence is that these effects depend strongly on the radiation polarization and the angle of incidence. The addressed restrictions on the second-order conductivities are given by the pointgroup operations and determine the experimental geometry. They are analyzed in the first part of this section. Afterwards, the existing experiments are introduced and discussed in the second part together with microscopic models. This discussion is extended by a short account on microscopic theory of some effects discussed in the literature but not observed so far.

Our analysis begins with $d c$ current generation, in order to demonstrate all important features of the nonlinear response, including an interplay between the spatial symmetry reduction and wavevector induced effects and sensitivity to the radiation helicity. Further second order effects, such as generation of an ac electric current giving rise to the harmonics generation or optical rectification, are discussed later on in Sections 3.4 and 3.5. The effect of $d c$ current generation is given by the second term in the right hand side of Eq. (2). As addressed above, the nonlinear conductivity $\sigma_{\alpha \beta \gamma}^{(2)}(\omega, \boldsymbol{q})$ has contributions due to both the reduced symmetry and radiation wavevector $\mathbf{q}$. Therefore, it can be conveniently decomposed in the sum of two parts yielding the $d c$ current in the form

$$
j_{\alpha}=\sigma_{\alpha \beta \gamma}^{(2)}(\omega, \boldsymbol{q}) E_{\beta} E_{\gamma}^{*}=\left[\sigma_{\alpha \beta \gamma}^{(2)}(\omega, 0)+\Phi_{\alpha \beta \gamma \mu}(\omega) q_{\mu}\right] E_{\beta} E_{\gamma}^{*}
$$

where linear in the wavevector $\boldsymbol{q}$ terms are taken into consideration, corresponding contribution is described by the fourth rank tensor $\Phi_{\alpha \beta \gamma \mu}(\omega)$. Such effects are related with the transfer of the photon momentum to the electrons. First we address the third-rank tensor $\sigma_{\alpha \beta \gamma}^{(2)}(\omega, 0)$, which describes the class of phenomena known as photogalvanic effects (PGE) [32-35,82-84] being present in noncentrosymmetric systems only. Therefore, in ideal graphene all photogalvanic effects are strictly forbidden by symmetry. However, in most of real structures PGE becomes possible, e.g. for the excitation in the vicinity of the edges, which locally reduce the symmetry, in the samples with ripples, or if the graphene layers are deposited on the substrate. Two types of photogalvanic effects, linear PGE and circular PGE, are known and are already observed in graphene [62]. The linear PGE is sensitive to the orientation of the radiation polarization plane, and is described by the symmetrical with respect to the interchange of $\beta \leftrightarrow \gamma$ part of $\sigma_{\alpha \beta \gamma}^{(2)}(\omega, 0)$. It is given by

$$
j_{\alpha} \propto E_{\beta} E_{\gamma}^{*}+E_{\gamma} E_{\beta}^{*}
$$

This symmetrized combination of electric field components is proportional to the linear combination of the Stokes parameters $S_{1}$ and $S_{2}$, see Eq. (6a). By contrast, the circular PGE requires angular momentum of photons and, correspondingly, given by the antisymmetric part of the tensor $\sigma_{\alpha \beta \gamma}^{(2)}(\omega, 0)$,

$$
j_{\alpha} \propto E_{\beta} E_{\gamma}^{*}-E_{\gamma} E_{\beta}^{*} \propto P_{\text {circ }}
$$

Here $P_{\text {circ }}$ is the degree of circular polarization of the radiation given by the Stokes parameter $S_{3}$, see Eq. (6b).

While the photogalvanic effects are possible only in the systems lacking an inversion center, the $d c$ current generation proportional to the radiation wavevector $\boldsymbol{q}$ and described by second term in Eq. (8) is allowed both in centrosymmetric and noncentrosymmetric media and, consequently, can take place in any graphene system. The fact, that the electric current can be caused by the momentum transfer from photons to electrons was recognized as early as in beginning of 1970 s and the effect was named as a photon drag effect [85,86]. Even earlier, in 1954 Barlow derived such a dc current in terms of ac (dynamic) Hall effect: The joint action of electric, $\boldsymbol{E}$, and magnetic, $\boldsymbol{B}$, fields of the radiation causes a steady-state current in the form $\boldsymbol{j} \propto[\boldsymbol{E} \times \boldsymbol{B}]$ [87]. These, at first glance, different mechanisms are related to the same phenomena, since for the plane wave in the form of Eq. (1) the complex amplitudes of electric and magnetic fields are coupled:

$$
\boldsymbol{B}(\omega, \boldsymbol{q})=\frac{1}{|\boldsymbol{q}|}[\boldsymbol{q} \times \boldsymbol{E}(\omega, \boldsymbol{q})],
$$

and dynamic Hall contribution $\propto E_{\beta} B_{\gamma}^{*}$ can be written in form of photon drag effect, i.e. $\propto q_{\delta} E_{\beta} E_{\gamma}^{*}$. Therefore usually, the terminology choice between the photon drag and dynamic Hall effects is determined by the microscopic treatment in terms of the number of photons absorbed (quantum mechanical picture-photon drag effect) or the action of electromagnetic fields (classical picture-dynamic Hall effect). While hereafter we equally use both terms, for the phenomenological consideration we prefer the term "photon drag" effect because the second term in Eq. (8) is proportional to the wavevector q. Similarly to the photogalvanic effect, the photon drag effect may take place in response to both linearly and circularly polarized radiation, which are described, respectively, by the symmetric and antisymmetric in $\beta \gamma \leftrightarrow \gamma \beta$ parts of the fourth-rank tensor $\Phi_{\alpha \beta \gamma}$. These effects are termed as linear and circular photon drag effects [88-91].

While point symmetry and, particularly, spatial inversion impose restrictions on the conditions of observation, polarization and incidence angle dependence of the effects, another important symmetry operation, namely, time reversal places 
additional limitations affecting the frequency dependence of the response. Electric current, $\boldsymbol{j}$, and radiation wavevector, $\boldsymbol{q}$, are odd at time reversal $\boldsymbol{j} \rightarrow-\mathbf{j}, \boldsymbol{q} \rightarrow-\boldsymbol{q}$ at $t \rightarrow-t$. The bilinear combinations of the field related with the linear polarization $E_{\beta} E_{\gamma}^{*}+E_{\beta}^{*} E_{\gamma}$ are invariant under time reversal. Therefore, the symmetric part of the nonlinear conductivity $\sigma_{\alpha \beta \gamma}^{(2)}(\omega, 0)$ describing linear photogalvanic effect is odd at time reversal, i.e. it contains odd powers of dissipative constants, i.e. scattering rates given by the reciprocal relaxation time $\tau^{-1}$ or absorption coefficient. By contrast, the nonlinear conductivity responsible for the linear photon drag effect is even at time reversal and contains even powers of dissipative constants. For current sensitive to the radiation helicity, i.e., circular photon drag and circular photogalvanic effects, the situation is just opposite. Now, the circular polarization changes its sign at time reversal, therefore the constants describing circular photogalvanic effect are even at time reversal, while constants describing circular drag effect are odd. Owing to different properties under time reversal, the radiation frequency dependences of the linear and circular photocurrents, as well as of photon drag and photogalvanic effects, are distinct, see below for details.

\subsection{Photon drag effects in a single layer graphene}

We shall start the consideration with the photon drag effect because this mechanism of the $d c$ current generation does not imply a symmetry reduction and can be present in any graphene sample. Moreover, the photon drag effect makes it possible to illustrate all facets of phenomenological analysis, including dependence on the incidence angle and effects sensitive to the photon helicity.

The consistent phenomenological theory of the photon drag effect in graphene layers has been developed in Refs. [59,62]. Disregarding the substrate, infinite homogeneous graphene layer is described by the centrosymmetric $D_{6 \mathrm{~h}}$ point group. It follows that the tensor $\Phi_{\alpha \beta \gamma \mu}$ has five linearly independent components, which give rise to corresponding contributions to the photocurrent. However, two of them are related to normal to the graphene layer component of electric field, $E_{z}$, or of the wavevector, $q_{z}$, and, in two-dimensional system like graphene, are much weaker compared to the others. Hereafter, we disregard these contributions for all effects which can be induced without taking into account $E_{z}$ and $q_{z}$. Such a model will be named strictly two-dimensional. Hence, for ideal graphene layer, the photocurrent is given by:

$$
\begin{aligned}
& j_{x}=T_{1} q_{x} \frac{\left|E_{x}\right|^{2}+\left|E_{y}\right|^{2}}{2}+T_{2} q_{x} \frac{\left|E_{x}\right|^{2}-\left|E_{y}\right|^{2}}{2}, \\
& j_{y}=T_{2} q_{x} \frac{E_{x} E_{y}^{*}+E_{x}^{*} E_{y}}{2}-\tilde{T}_{1} q_{x} P_{\text {circ }} \hat{e}_{z}\left(\left|E_{x}\right|^{2}+\left|E_{y}\right|^{2}\right) .
\end{aligned}
$$

Here $\hat{\boldsymbol{e}}=\left(\hat{e}_{x}, \hat{e}_{y}, \hat{e}_{z}\right) \equiv \boldsymbol{q} /|q|$ is a unit vector in the direction of light propagation, and we introduced the coordinate frame with axes $x$ and $y$ chosen in the graphene plane, $z$ being the sample normal and assume that radiation is incident in the $(x z)$ plane, therefore, $q_{y} \equiv 0$, see Fig. 2. Such a choice of the coordinates is adjusted to convenient experimental geometry where the current is investigated along and normal to the incidence plane. We note that field combinations in Eqs. (10) directly correspond to Stokes parameters, Eqs. (6). Constants $T_{1}$ and $T_{2}$ describe linear photon drag effect. The specific feature of graphene compared to three-dimensional cubic semiconductors and simple metals is the presence of the circular photon drag effect given by the constant $\tilde{T}_{1}$. A further peculiarity of the photon drag effect in graphene comes from its twodimensional nature: Here the photon drag current is present under oblique incidence only and its direction changes upon reversal of the incidence angle. ${ }^{6}$ In the presence of substrate or adatoms deposited on one side of the sample, the symmetry of graphene reduces to the noncentrosymmetric group $C_{6 \mathrm{v}}$. In such a case, the equivalence of the $z$ and $-z$ directions is removed. Analysis shows that the form of Eqs. (10) remains the same for noncentrosymmetric graphene described by the $C_{6 \mathrm{v}}$ point symmetry group. While the functional behavior does not change, the effect may originate from diverse microscopic mechanisms and, consequently, be characterized by different magnitudes of the corresponding constants in Eqs. (10).

It follows from Eqs. (10a), (10b) that the photon drag current contains, in general, three contributions illustrated in Fig. 2, panels (a)-(c). First one, schematically illustrated in Fig. 2(a) results in the photocurrent, which flows along the light incidence plane. Two other effects are caused by the reduced symmetry of the system and exhibit a specific polarization dependence described by the combinations of electric field components $E_{x}, E_{y}$ in Eqs. (10). The terms proportional to $T_{2}$ are sensitive to the linear polarization and yield photocurrent components (i) in the plane of incidence and (ii) perpendicular to the incidence plane, see Fig. 2(b). By contrast, the current proportional to $\tilde{T}_{1}$ is due to transfer of both light linear and angular momenta to electrons and reverses its sign by changing photon helicity. This is the circular photon drag effect or, as addressed in the previous section, circular ac Hall effect, which appears in the transverse to the light propagation plane geometry, see Fig. 2(c).

In experiments described below, see Section 5 , the polarization state of incident radiation was controlled by half- or quarter-wave plates. In the former case of linearly polarized radiation, its helicity $P_{\text {circ }} \equiv S_{3}=0$ and, hence, only the components of the photocurrent proportional to $T_{1}$ and $T_{2}$ are excited, see Eqs. (10). For not too large incidence angles $\theta_{0}$,

\footnotetext{
6 We note that in some systems with reduced symmetry due to e.g., ripples, strain or terraces, the photon drag effect may also be allowed at normal incidence. However, these contributions are expected to be very small, as they are proportional to two small factors: the photon wavevector and the degree of asymmetry. Hence, such effects are out of scope of present review.
} 
a

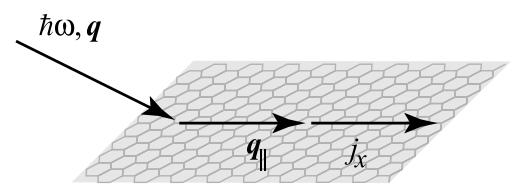

b

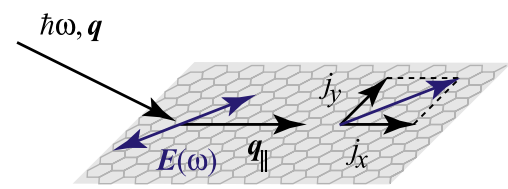

C

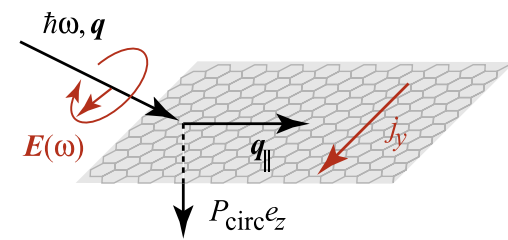

d

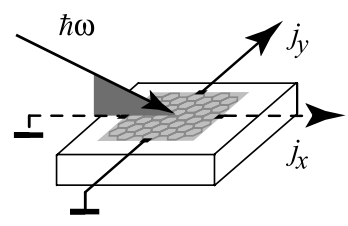

e $\hbar \omega$
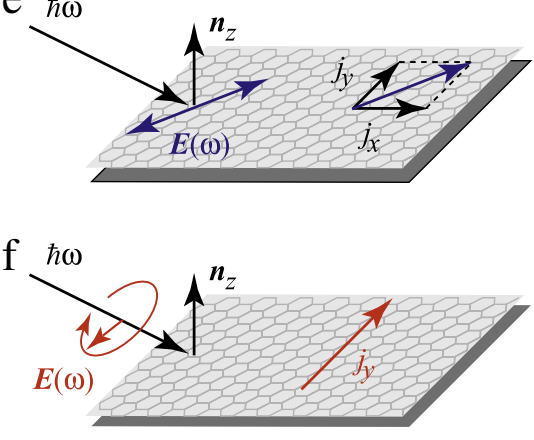

Fig. 2. Schematic illustration of the possible contributions to the photon drag and photogalvanic effects. Panels (a)-(b): linear and (c) circular photon drag effects, respectively [see Eqs. (10) and (11)]. Note that these figures are relevant both to ideal graphene and graphene on the substrate. Panels (e)-(f): linear and circular photogalvanic effects allowed by symmetry in graphene samples with structure inversion asymmetry, i.e. deposited on substrates [see Eqs. (12) and (13)]. Panel (d) shows a relevant experimental geometry. After [62].

where $\sin \theta_{0} \approx \theta_{0}$ and $\cos \theta_{0} \approx 1$ (see Ref. [59] for discussion of the arbitrary incidence angle), the component of the current in the radiation incidence plane, $j_{x}$, is given by the sum of the polarization-independent contribution [ $T_{1}$ term in Eq. (10a)] and the contribution excited by polarized light being proportional to $S_{1} \propto \cos 2 \alpha\left(T_{2}\right.$ term). The current component normal to the incidence plane, $j_{y}$, is excited by the polarized radiation only, it is proportional to the Stokes parameter $S_{2}$, which varies as $\sin 2 \alpha$. In the geometry with a quarter-wave plate one obtains elliptically polarized radiation and, in addition to the contributions described above, one can generate current sensitive to the radiation helicity. Here the perpendicular to the incidence plane component of the photocurrent, $j_{y}$, can be presented as a superposition of the contributions excited by linearly and circularly polarized light, being described by the linear combination of the Stokes parameters $S_{2}$ and $S_{3}$, respectively. For the particular choice of the angles accepted by Eq. (7b) it is described by

$$
j_{y}=j_{A} \sin 2 \varphi+j_{B} \sin 4 \varphi,
$$

where $j_{A}=A|\boldsymbol{E}|^{2} \theta_{0}$ and $j_{B}=B|\boldsymbol{E}|^{2} \theta_{0}$ are the magnitudes of circular and linear contributions, respectively. The current in the incidence plane is given by the superposition of terms $\propto S_{1}$ and $S_{2}$, namely,

$$
j_{x}=j_{B} \cos 4 \varphi+j_{C}
$$

with $j_{C}=C|E|^{2} \theta_{0}$. Full expressions for the photocurrent components in the terms of Stokes parameters under oblique incidence are presented in Ref. [59].

\subsection{Photogalvanic effects in a single layer graphene}

Appearance of the photogalvanic effects implies that the spatial inversion symmetry is broken. Such a situation for flat infinite sample is realized for the graphene layer deposited on the substrate or if adatoms predominantly are present on one surface of the material. The symmetry of graphene on a substrate is $C_{6 \mathrm{v}}$ and the PGE for the oblique incidence in the ( $x z$ ) plane is described for not too large incidence angles by

$$
\begin{aligned}
& j_{x}=\chi_{l} \frac{E_{x} E_{z}^{*}+E_{x}^{*} E_{z}}{2}, \\
& j_{y}=\chi_{l} \frac{E_{y} E_{z}^{*}+E_{y}^{*} E_{z}}{2}+\chi_{c} P_{\text {circ }} \hat{e}_{x}\left(\left|E_{x}\right|^{2}+\left|E_{y}\right|^{2}\right),
\end{aligned}
$$

where two independent parameters, $\chi_{l}$ and $\chi_{c}$, describe linear and circular PGE, respectively. Similar to the photon drag effect, the PGE can be generated at oblique incidence only, however, in this case it comes from the necessity to provide $z$-component of electric field, rather than in-plane photon wavevector $\mathbf{q}$, see Eqs. (12). Another specific feature of the PGE compared to the photon drag effect is that it cannot be generated by unpolarized radiation. Linear and circular PGE currents given by $\chi_{l}$ and $\chi_{c}$ are schematically shown in Fig. 2(e) and (f), respectively. It follows from Eqs. (12a), (12b) that the linear current flows along the projection of the electric field onto the sample plane and, therefore, in general, it has both components along, $j_{x}$, and normal, $j_{y}$, to the light incidence plane. By contrast, circular photocurrent flows perpendicularly 
to the radiation incidence plane, i.e., along $y$ axis in the chosen geometry. We note, that the dependence of the photogalvanic effect on the polarization state of light and, consequently on the wave plate orientation angles $\alpha$ and $\varphi$ is indistinguishable from that of the photon drag effect, see Eq. (11). In particular, for small incidence angles

$$
\begin{aligned}
& j_{x}=j_{B^{\prime}} \cos 4 \varphi+j_{C^{\prime}}, \\
& j_{y}=j_{A^{\prime}} \sin 2 \varphi+j_{B^{\prime}} \sin 4 \varphi,
\end{aligned}
$$

where $j_{A^{\prime}}=A^{\prime}|\boldsymbol{E}|^{2} \theta_{0}, j_{B^{\prime}}=B^{\prime}|\boldsymbol{E}|^{2} \theta_{0}$ and $j_{C^{\prime}}=C^{\prime}|E|^{2} \theta_{0}$ with constants $A^{\prime}, B^{\prime}$ and $C^{\prime}$ are circular $\left(j_{A^{\prime}}\right)$ and linear $\left(j_{B^{\prime}}, j_{C^{\prime}}\right)$ photocurrent components. It follows from Eqs. (12) the parameters $j_{B^{\prime}}$ and $j_{C^{\prime}}$ are interrelated according to $j_{C^{\prime}}=-j_{B^{\prime}}$ for $s$ polarization at $\varphi=0$, while $j_{C^{\prime}}=3 j_{B^{\prime}}$ for $p$ polarization at $\varphi=0$ [92]. We emphasize, however, that in the case of photogalvanic effects the microscopic sense of the parameters $A^{\prime}, B^{\prime}$ and $C^{\prime}$ is distinct from that of the corresponding coefficients $A, B$ and $C$ for the photon drag effect, since PGE is related to the absence of an inversion center.

The requirement of $z$-component of the field diminishes PGE in graphene, since strictly two-dimensional carriers are almost unaffected by $E_{z}$. This is in contrast to the conventional semiconductor two-dimensional systems like, e.g., quantum wells and heterojunctions, where in spite of the fact that there is no carrier motion in $z$ direction the wavefunction is extended over many atomic layers and can be easily affected by an electric field. Due to the fact that the polarization behaviors of PGE and photon drag are similar, the PGE is usually masked by the stronger drag effect in graphene. Thus, the observation of PGE is most likely in conditions where the photon drag effect is reduced, e.g. at high radiation frequencies, see Section 5.3 for details.

The situation changes, however, in graphene structures of lower symmetry, which is reduced, e.g., due to asymmetric ripples, curvatures, edges, terraces, etc. Here new contributions to PGE appear, which do not require the action of $z$ component of electric field on electrons. In particular, excitation of edges represents the natural route of the symmetry reduction. Disregarding the microscopic structure of the edge and presence of the substrate, we deal with the point symmetry $C_{2 v}$ having the two-fold rotation axis perpendicular to the edge and lying in the sample plane. Corresponding additional to Eqs. (12) contributions to the photocurrent are given by

$$
j_{y}=R_{l} \frac{E_{x} E_{y}^{*}+E_{x}^{*} E_{y}}{2}+R_{c} P_{\text {circ }} \hat{e}_{z}\left(\left|E_{x}\right|^{2}+\left|E_{y}\right|^{2}\right),
$$

where the edge is assumed to be along $y$ axis, and two constants, $R_{l}$ and $R_{c}$, describing the linear and circular edge PGE are introduced. Comparing Eqs. (6) and (14) we see that the polarization dependences of these contributions are given by the Stokes parameters $S_{2}$ and $S_{3}$, respectively. We emphasize that edge photogalvanic effect can be observed even for the normal incidence of radiation where the photon drag is forbidden. Obviously, it is sensitive to the quality and microstructure of the edge and provides an experimental access to this important parameters.

A further reduction of edge symmetry may come from the fact, that the edge orientation of the graphene layer is maintained with an atomic accuracy and its direction differs from high-symmetry ones. In this case, the point symmetry of the system lowers down to $C_{\mathrm{s}}$ (if the substrate is absent) or further to $C_{1}$ (with allowance for the substrate). In both cases even unpolarized radiation at normal incidence can cause the photocurrent flowing along the edge, and the direction of the current is determined by the microscopic structure of the edge.

Besides edge photogalvanic effects, the symmetry reduction compared to ideal graphene layer may also come from the other factors both natural and produced on purpose, e.g., terraces, strain, ripples, artificial lateral superlattices etc. The symmetry of the system can be lowered depending on the specifics of the perturbation. In all these cases, the current at normal incidence can be generated by linearly, circularly or even unpolarized light, its direction and particular polarization dependence indicates the symmetry of perturbation (see e.g. [93,94], where photogalvanic effects in perturbed graphene with lateral superlattice were addressed theoretically).

\subsection{Photogalvanic and photon drag effects in multilayer graphene}

An important issue of graphene structures is the possibility to arrange several atomic layers one on the top of the other. The striking examples of these systems are graphene bilayers and trilayers whose physical properties attract now a great interest [95-101]. In these kinds of systems the response to $z$ component of electric field required for PGE current can be enhanced as compared with that in the single layer graphene because each additional layer allows more freedom for electron to move along the sample normal. Therefore, it is expected to make the most pronounced impact on the photogalvanic effect by affecting the microscopic processes of the current formation.

Hence, in multilayer systems the coefficients $\chi_{l}$ and $\chi_{c}$ in Eq. (12) describing linear and circular PGE under oblique incidence may be strongly modified and enhanced. At the same time, the dominant contribution to the photon drag effect in the multilayers is given by Eqs. (10) and the constants $T_{1}, T_{2}$ and $\tilde{T}_{1}$ differ from those in a single layer due to the modification of electron energy spectrum and scattering processes.

Besides, the multilayer stacking may contribute to the symmetry reduction and may give rise to the novel photogalvanic effects inherent in multilayer systems only. The point symmetry of graphene $N$-layers depends on the stacking type and on the layer number, $N$. Here we consider only two "natural" orderings: the rhombohedral stacking (ABCABC...), described by the point symmetry group $D_{3 \mathrm{~d}}$, which contains an inversion center [102], and the Bernal one (ABAB...). In the latter case, 

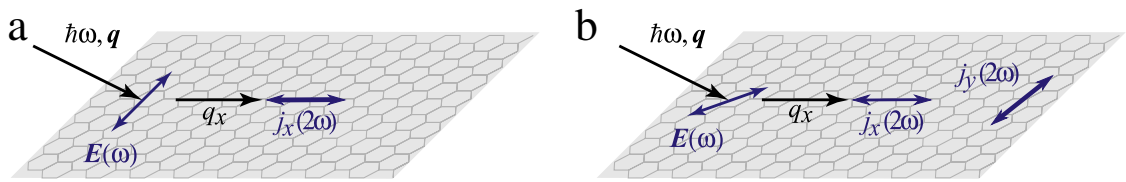

Fig. 3. Schematic illustration of the second harmonic generation described by Eqs. (16). Panel (a) shows current component in the incidence plane at a double frequency, $j_{x}(2 \omega)$ excited by fundamental frequency light polarized along $y$-axis, (b) shows both contributions to the second harmonic current parallel and perpendicular to the radiation incidence plane for general direction of $\boldsymbol{E}(\omega)$. After [106].

the point symmetry is described by either $D_{3 \mathrm{~d}}$ group (for even $N$ ), which contains an inversion center, or by $D_{3 \mathrm{~h}}$ group (for odd $N>1$ ) [103]. For odd $N>1$ the CPGE is also symmetry forbidden for ideal system, however, the linear photogalvanic current becomes possible even for normal incidence ${ }^{7}$

$$
j_{x}=\chi_{l}^{\prime}\left(\left|E_{x}\right|^{2}-\left|E_{y}\right|^{2}\right), \quad j_{y}=-\chi_{l}^{\prime}\left(E_{x} E_{y}^{*}+E_{y} E_{x}^{*}\right),
$$

and described by a single parameter $\chi_{l}^{\prime}$. Here $x$ axis is chosen along one of $C_{2}$ axes in the sample plane. Finally, bulk graphite is described by $D_{6 \mathrm{~h}}$ point symmetry group, which contains spatial inversion. Hence, in this material the photogalvanic effects are forbidden and only photon drag current is possible. Like in case of monolayers, the presence of the substrate or the top gate can reduce the symmetry of the multilayer graphene system and give rise to the photocurrents, which are forbidden in monolayers. For example, the symmetry of the bilayer deposited on the substrate reduces from $D_{3 \mathrm{~d}}$ to $C_{3 \mathrm{v}}$ and the linear photocurrent described phenomenologically by Eqs. (15) becomes possible.

\subsection{Second harmonic generation}

Phenomenological analysis of the second harmonic generation (SHG) in graphene and graphene-based systems is quite analogous to that of the $d c$ photocurrents, see Ref. [106] for details. The important distinctions are as follows: (i) unlike $d c$ current generation where the current flows only in graphene plane, the ac current associated with the harmonic generation can have normal to the graphene plane component, and (ii) the second harmonic is described by the quadratic combinations $E_{\beta} E_{\gamma}$ in contrast to the $d c$ current formation proportional to the $E_{\beta} E_{\gamma}^{*}$. Consequently, the nonlinear conductivity $\sigma_{\alpha \beta \gamma}^{\left(2^{\prime}\right)}$ is invariant under the permutation of the last two subscripts. It follows that for unpolarized and linearly polarized radiation the symmetry description of the SHG is similar to that of the linear photon drag and photogalvanic effects: In the very same way, there are contributions to the harmonic related with the absence of an inversion center in the medium and with the photon wavevector q. In strictly two-dimensional model for an ideal single-layer sample the current at a double frequency is described by two linearly independent complex constants $Q_{1}$ and $Q_{2}$ as

$$
\begin{aligned}
& j_{x}(2 \omega, 2 \boldsymbol{q})=Q_{1} q_{x}\left(E_{x}^{2}+E_{y}^{2}\right)+Q_{2}\left[q_{x}\left(E_{x}^{2}-E_{y}^{2}\right)+2 q_{y} E_{x} E_{y}\right], \\
& j_{y}(2 \omega, 2 \boldsymbol{q})=Q_{1} q_{y}\left(E_{x}^{2}+E_{y}^{2}\right)+Q_{2}\left[q_{y}\left(E_{y}^{2}-E_{x}^{2}\right)+2 q_{x} E_{x} E_{y}\right] .
\end{aligned}
$$

Comparing these expressions with Eqs. (10a) and (10b) for linear photon drag effect we see that, as addressed above, the electric field and wavevector dependences of these effects are just the same. Although second harmonic can be generated by unpolarized, linearly polarized or even circularly polarized radiation, no contribution sensitive to the radiation helicity to the second harmonic current is possible owing to the fact that the quadratic combinations $E_{\beta} E_{\gamma}$ in Eqs. (16) are not sensitive to the radiation helicity. Therefore, analogues of the helicity driven $d c$ current given by bilinear contributions $E_{\beta} E_{\gamma}^{*}-E_{\beta}^{*} E_{\gamma}$ in Eqs. (10), (12), (14) are absent for the SHG.

Fig. 3 schematically shows the geometry of the second harmonic generation and the response at a double frequency, $2 \omega$. For ideal graphene sample the second harmonic is excited only at the oblique incidence of radiation and is caused by the photon wavevector $\mathbf{q}$. For instance, for $q_{x} \neq 0, q_{y}=0$, there is a component of the in-plane oscillating current $\mathbf{j}(2 \omega)$ parallel to the light incidence plane described by $\left(Q_{1}+Q_{2}\right) q_{x} E_{x}^{2}+\left(Q_{1}-Q_{2}\right) q_{x} E_{y}^{2}$ [Fig. 3(a)]. Additionally, there is a contribution, $2 Q_{2} q_{x} E_{x} E_{y}$, perpendicular to the incidence plane, see Fig. $3(\mathrm{~b})$.

Since the nonlinear conductivities $\sigma_{\alpha \beta \gamma}^{(2)}$ describing linear photon drag/photogalvanic effects and $\sigma_{\alpha \beta \gamma}^{\left(2^{\prime}\right)}$ responsible for the SHG, transform under symmetry operations in the same way, the phenomenological analysis of the effects of substrate, adatoms, ripples, edges, multilayer stacking, etc. on photocurrents, presented above, holds also for the second harmonic generation.

\subsection{Optical rectification}

Optical rectification complements the class of the discussed above second order effects resulting in $d c$ or ac electric current. It refers to the formation of the steady state dielectric polarization $\boldsymbol{P}$ in response to the radiation propagating in the media [107]. While the point symmetry restrictions on the optical rectification effect are the same as for the $d c$ current

\footnotetext{
7 The electron states in the single valley $\boldsymbol{K}$ or $\boldsymbol{K}^{\prime}$ of graphene monolayer possess $D_{3 \mathrm{~h}}$ point symmetry, hence, under the normal incidence the linearly polarized light can induce the valley-orbit currents described in Refs. [104,105].
} 
generation and described by Eqs. (2), (8), $\boldsymbol{P}$ does not change its sign under the time reversal. Thus the constants responsible for the optical rectification and $d c$ current generation have different properties at time reversal. As a consequence, in contrast to photogalvanic or photon drag effects, the optical rectification gives rise to the electric current during the transient process only, when the illumination is turned on or off [34,108]

$$
\boldsymbol{j}=\frac{d \boldsymbol{P}}{d t} \text {. }
$$

Accordingly, microscopic mechanisms of the optical rectification, photon drag and photogalvanic effects are different. In particular, in contrast to the photon drag and photogalvanic effects, optical rectification does not require optical absorption and may take place in the transparency region.

To complete the picture, we note that the most general case of two incident waves with frequencies $\omega_{1}$ and $\omega_{2}$ (wavevectors $\boldsymbol{q}_{1}$ and $\boldsymbol{q}_{2}$ ) can also be considered. In this situation, the current or polarization response contains the nonlinear contributions corresponding to the sum and difference of the frequencies, $\omega_{1} \pm \omega_{2}$, and wavevectors, $\mathbf{q}_{1} \pm \mathbf{q}_{2}$,

$$
j_{\alpha} \propto E_{\beta}\left(\omega_{1}, \boldsymbol{q}_{1}\right) E_{\gamma}\left(\omega_{2}, \boldsymbol{q}_{2}\right) \mathrm{e}^{-\mathrm{i}\left(\omega_{1}+\omega_{2}\right) t+\mathrm{i}\left(\boldsymbol{q}_{1}+\boldsymbol{q}_{2}\right) \boldsymbol{r}},
$$

and

$$
j_{\alpha} \propto E_{\beta}\left(\omega_{1}, \boldsymbol{q}_{1}\right) E_{\gamma}^{*}\left(\omega_{2}, \boldsymbol{q}_{2}\right) \mathrm{e}^{-\mathrm{i}\left(\omega_{1}-\omega_{2}\right) t+\mathrm{i}\left(\boldsymbol{q}_{1}-\boldsymbol{q}_{2}\right) \boldsymbol{r}},
$$

respectively, giving rise to the three-wave mixing effects. Note that for $\omega_{1}=\omega_{2}$ in Eqs. (18a) we obtain second harmonic and $d c$ current generation described above. Phenomenological analysis of these effects can be carried out in a way similar to the description of the photon drag and photogalvanic effects.

\section{Second order effects: theoretical background}

Microscopic theory of second order effects in graphene was discussed in a number of works considering classical and quantum regimes of light-matter interaction $[40,58-61,74,106]$. In order to illustrate the appearance of the second-order nonlinear effects in ideal graphene we first consider the classical range of radiation frequencies given by Eq. (5d) and describe the electron dynamics in the framework of the second Newton law:

$$
\frac{d \boldsymbol{p}}{d t}+\frac{\boldsymbol{p}}{\tau}=e \boldsymbol{E}(\boldsymbol{r}, t)+\frac{e}{c}[\boldsymbol{v} \times \boldsymbol{B}(\boldsymbol{r}, t)] .
$$

The approach is a standard way widely used for other nonlinear media [109,110], the specificity of graphene comes from the unusual relation between the momentum $\boldsymbol{p}$ and velocity $\boldsymbol{v}$,

$$
\boldsymbol{v}=v \boldsymbol{p} / p
$$

and details of effective friction force, $-\boldsymbol{p} / \tau$, acting on the electron due to the scattering processes. Eq. (19) contains both the force acting from the electric field of the radiation and the Lorentz force caused by the magnetic field of the wave.

Eq. (19) can be solved by iterations in the strength of electro-magnetic field. At a first stage we determine the linear response of electron on the oscillating electric field. The momentum oscillations are written as

$$
\tilde{\boldsymbol{p}}(t)=\frac{e \tau \boldsymbol{E}_{\|} \mathrm{e}^{-\mathrm{i} \omega t}}{1-\mathrm{i} \omega \tau}+\text { c.c. },
$$

where $\boldsymbol{E}_{\|}$is the field component in the plane of the graphene monolayer. The second stage of calculations is to determine the nonlinear response, induced by the forces in the right hand side of Eq. (19). It contains two contributions: One is related to the action of magnetic field, in which case the second-order correction to the electron momentum is caused by the Lorentz force. The other one results from the coordinate dependence of the electric field and does not require magnetic field at all. Below we consider both effects one by one and start with the response to the magnetic field. In this case, the steady state momentum is given by

$$
\overline{\boldsymbol{p}}=\frac{\overline{e \tau}}{c}[\tilde{\boldsymbol{v}} \times \boldsymbol{B}(t)] .
$$

Here the overline denotes the time-averaging, $\tilde{\boldsymbol{v}}$ is the oscillatory part of the velocity determined by Eqs. (20) and (21). The coordinate dependence of the fields can be neglected. In the same way, the oscillations of the momentum at a double frequency (second harmonic generation) are given by the similar to Eq. (22) expression:

$$
\tilde{\tilde{\boldsymbol{p}}}=\frac{e \tau}{(1-2 \mathrm{i} \omega \tau) c}[\tilde{\boldsymbol{v}} \widetilde{\times \boldsymbol{B}(t)}]
$$

Here wide tilde means taking the contribution oscillating at $2 \omega$.

To illustrate the microscopic origin of the second-order responses we consider the photon drag effect as an example. The steady-state momentum $\overline{\boldsymbol{p}}$ in Eq. (22) corresponds to the $d c$ current flow. Physically, it is related with the electron drift caused by the crossed electric and magnetic fields of the wave. Basic physics of this effect is illustrated in Fig. 4(a). We assume 


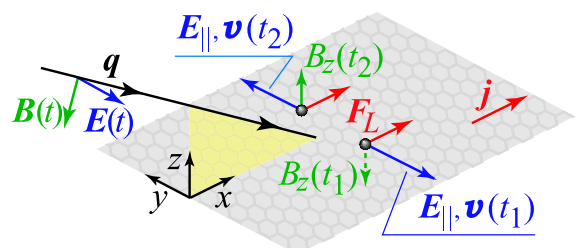

(a) Linearly polarized radiation.

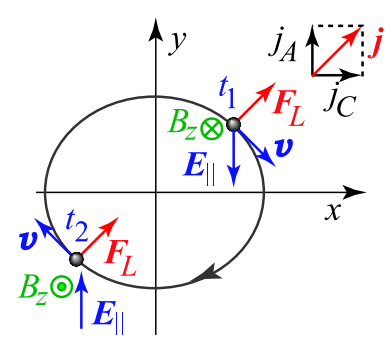

(c) $\sigma^{+}$, right-handed radiation.

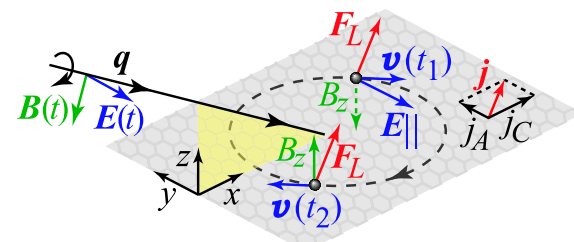

(b) $\sigma^{+}$, right-handed radiation.

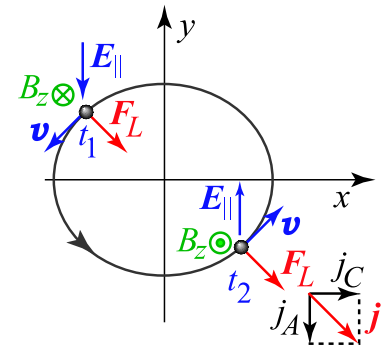

(d) $\sigma^{-}$, left-handed radiation.

Fig. 4. Schematic illustration of the dynamic Hall effect. For simplicity we assume positively charged carriers, i.e., holes. $\boldsymbol{E}_{\|}, B_{z}$ and $\boldsymbol{v}$ denote the in-plane components of electric field of the radiation, $z$-component of the magnetic field and the electron velocity, induced by electric field, respectively. These vectors are shown for two time moments, $t_{1}$ and $t_{2}$, corresponding to half-periods of the field oscillations. Microscopically, action of these fields results in the Lorentz force, $\boldsymbol{F}_{L}$, and, correspondingly, $d c$ current, $\boldsymbol{j}$, see text for details. (a) Linear effect. (b) Circular effect, $\sigma^{+}$radiation. The dashed orbit represents the hole elliptical trajectory caused by the ac $\boldsymbol{E}$-field. (c) and (d) show top view of panel (b) for $\sigma^{+}$and $\sigma^{-}$radiation, respectively. After [58].

the oblique incidence of the radiation in the $(x z)$ plane, and for the sake of illustration, consider the case of $s$-polarized radiation, where the electric field oscillates in the sample plane. At one of half-periods of oscillation, say, at time moment $t_{1}$, the radiation electric and magnetic fields acting on charge carrier result in a Lorentz force and, consequently, carrier drift in the direction of the light propagation (longitudinal current). At the second half of period $t_{2}$ both fields reverse, hence, the drift direction remains. The resulting current is so called dynamic or ac Hall effect, which was considered by H.M. Barlow [87]. The mechanism of the second-order response due to the joint action of electric and magnetic fields is named $E B$-mechanism. In quantum mechanical approach it corresponds to the magneto-dipole transitions.

While the longitudinal current is expected for unpolarized and even circularly polarized radiation, the appearance of the photon helicity dependent current is not obvious. However, as shown in Ref. [58] such current indeed emerges if one takes into account the effect of retardation between the electric field $\boldsymbol{E}$ and the instant velocity of charge carrier $\boldsymbol{v}$, being most pronounced for $\omega \tau \sim 1$. The model picture of the circular ac Hall effect is presented in Fig. 4(b)-(d). For circularly polarized radiation, the electric field rotates around the wavevector $\mathbf{q}$, sketched in Fig. 4(b) for $\sigma_{+}$circularly polarized light. Now, instead of linear oscillations, the carriers follow the elliptic orbit. At an instant of time $t_{1}$, the carrier is accelerated by the in-plane component $\boldsymbol{E}_{\|}$of the ac electric field. At the same time, the carrier with velocity $\boldsymbol{v}$ is subjected to the out-of-plane magnetic field component $\boldsymbol{B}_{z}$. Note, that the velocity $\boldsymbol{v}$ does not instantaneously follow the actual $\boldsymbol{E}_{\|}$-field direction due to retardation: There is a phase shift equal to $\arctan (\omega \tau)$ between the electric field and the electron velocity $\boldsymbol{v}$. The effect of retardation, well known in the Drude-Lorentz theory of high frequency conductivity, results in an angle between the velocity $\boldsymbol{v}$ and the electric field direction $\boldsymbol{E}_{\|}$, which depends on the value of $\omega \tau$. The resulting Lorentz force $\boldsymbol{F}_{L}=e / c\left[\boldsymbol{v} \times \boldsymbol{B}_{z}\right]$ generates a Hall current $\boldsymbol{j}$, also shown in Fig. 4. Half a period later at $t_{2}=t_{1}+T / 2$, both $\boldsymbol{v}$ and $\boldsymbol{B}_{z}$ get reversed so that the direction of $\boldsymbol{F}_{L}$ and, consequently, the current $\boldsymbol{j}$ stay the same. The oscillating magnitude and direction of $\boldsymbol{B}_{z}$ along the trajectory lead to a periodical modulation of the Lorentz force with nonzero average causing a nonzero time-averaged Hall current with fixed direction. If, as shown in Fig. 4(d), the light helicity is reversed, the electric field rotates in the opposite direction and, thus, the charge carrier reverses its direction. Hence, owing to retardation, the $y$-component of $\boldsymbol{F}_{L}$ at $t_{1}$ and $t_{2}$ is inverted. Consequently the polarity of the transverse, time-averaged Hall current changes. We stress that the origin of the circular ac Hall effect is related with the retardation, which is very important if $\omega \tau \sim 1$. Such condition is readily realized for the state-of-the-art graphene samples at $\mathrm{THz}$ frequency range.

Now we turn to another mechanism of the second order response. As it follows from Eq. (19) this contribution comes from the fact that the momentum oscillations of an electron given by Eq. (21) result in the oscillations of its coordinate, $\tilde{\boldsymbol{r}}(t)$. The electric force acting on electron depends on its position owing to the coordinate-dependence of $\boldsymbol{E}$, corresponding contribution has the form [110]

$$
e \boldsymbol{E}_{\|} \mathrm{e}^{\mathrm{i} \boldsymbol{q} \boldsymbol{r}-\mathrm{i} \omega t} \approx e \mathrm{i}[\boldsymbol{q} \tilde{\boldsymbol{r}}(t)] \boldsymbol{E}_{\|} \mathrm{e}^{-\mathrm{i} \omega t}
$$

and its time average results in the steady-state response, while its second temporal harmonic gives rise to the second harmonic generation. This mechanism named as $q E^{2}$ mechanism corresponds to the quadrupole transitions in a quantum approach. 
The consistent theory of the second order response in the classical frequency range is developed in the framework of Boltzmann kinetic equation for the position $\boldsymbol{r}$, momentum $\boldsymbol{p}$ and time $t$ dependent electron distribution function:

$$
\frac{\partial f}{\partial t}+\boldsymbol{v} \frac{\partial f}{\partial \boldsymbol{r}}+e\left(\boldsymbol{E}+\frac{1}{c}[\boldsymbol{v} \times \boldsymbol{B}]\right) \frac{\partial f}{\partial \boldsymbol{p}}=Q\{f\},
$$

where $Q\{f\}$ is the collision integral. Eq. (24) takes into account the action of electric and magnetic fields of radiation and is solved iteratively in the field amplitudes. The details of calculations are presented in Refs. $[58,59,106]$. Corresponding results of calculations, comparison with available experimental data, and the extensions of treatment to cover quantum range of frequencies and to include the symmetry reduction owing to sample edges or substrate are reviewed below together with experimental results.

\section{Second order effects: experiment and theory}

\subsection{Second harmonic generation}

\subsubsection{Microscopic theory}

The SHG theory in graphene was presented in several works $[50,74,106]$. Ref. [74] deals with symmetry arguments and effects of radiation propagation in multilayer graphene-based systems. In the works $[50,106]$ both quantum mechanical and classical regimes were discussed. The approaches of Refs. $[74,106]$ agree for the intermediate frequencies $\tau^{-1} \ll \omega \ll E_{F} / \hbar$. Here we follow Ref. [106] and present the results of calculations for the constants $Q_{1}$ and $Q_{2}$ describing two independent contributions to the second harmonic current $\boldsymbol{j}(2 \omega, 2 \boldsymbol{q}) \propto Q_{1}, Q_{2}$, see Eqs. (16). These calculations, based on Boltzmann equation and describe the classical frequency range, yield

$$
\begin{aligned}
& Q_{1}=-\frac{e^{3} v^{4}}{2 \omega} \sum_{\boldsymbol{k}} \tau_{1, \omega} f_{0}^{\prime}\left[\frac{\tau_{1,2 \omega}}{\varepsilon_{k}}\left(3+\mathrm{i} \omega \tau_{2, \omega}\right)+\left(1-\mathrm{i} \omega \tau_{2, \omega}\right) \frac{\mathrm{d} \tau_{1,2 \omega}}{\mathrm{d} \varepsilon_{k}}\right], \\
& Q_{2}=\frac{e^{3} v^{4}}{2 \omega} \sum_{\boldsymbol{k}} \tau_{1, \omega} f_{0}^{\prime}\left\{\frac{\tau_{1,2 \omega}}{\varepsilon_{k}}\left(1+4 \mathrm{i} \omega \tau_{2,2 \omega}\right)-\frac{\mathrm{d}}{\mathrm{d} \varepsilon_{k}}\left[\tau_{1,2 \omega}\left(1-2 \mathrm{i} \omega \tau_{2,2 \omega}\right)\right]\right\} .
\end{aligned}
$$

Here $f_{0}^{\prime}=d f_{0} / d \varepsilon$,

$$
\tau_{n, \omega}=\frac{\tau_{n}}{1-\mathrm{i} \omega \tau_{n}}, \quad(n=1,2),
$$

with $\tau_{1}$ and $\tau_{2}$ being the momentum and momentum alignment relaxation times, respectively and the condition $\hbar \omega \ll E_{F}$ is assumed. Using the obtained ac second harmonic current $\boldsymbol{j}(2 \omega, 2 \boldsymbol{q})$ and Maxwell equation

$$
\Delta \boldsymbol{A}(\boldsymbol{r}, t)+\frac{4 \omega^{2}}{c^{2}} \boldsymbol{A}(2 \omega)=-\frac{4 \pi}{c} \mathrm{e}^{2 \mathrm{i} \boldsymbol{q}_{\|} \rho-2 \mathrm{i} \omega t} \delta(z) \boldsymbol{j}(2 \omega)+\text { c.c. },
$$

we obtain the vector potential $\boldsymbol{A}(\boldsymbol{r}, t)$ of emitted radiation. Here $\boldsymbol{q}_{\|}=\left(q_{x}, q_{y}\right)$ is the projection of radiation wavevector onto the sample plane $z=0$. Note, that the current oscillating at a double frequency, $\boldsymbol{j}(2 \omega)$, is proportional to the square of incident electric field, i.e. to the intensity of the fundamental harmonic. As a result, the intensity of the second harmonic is proportional to the fourth power of the incident electric field or the square of the fundamental harmonic intensity.

In the static limit, $\omega \rightarrow 0$, the coefficients $Q_{1}$ and $Q_{2}$ are real and diverge as $1 / \omega$, but the net current $\boldsymbol{j}(2 \omega, 2 \boldsymbol{q})$ remains finite due to factors $\propto \boldsymbol{q}$ in Eqs. (16). Coefficients $Q_{1}$ and $Q_{2}$ become, up to common factor, equal to the constants $T_{1}$ and $T_{2}$ describing linear photon drag effect, see Eqs. (10) and (29) below, because at $\omega=0$ responses at zero and double frequencies are indistinguishable. At high frequencies, $\omega \tau_{1} \gg 1, \omega \tau_{2} \gg 1$, parameters $Q_{1}$ and $Q_{2}$ are proportional to $1 / \omega^{3}$, hence, current density decays as $1 / \omega^{2}$.

A remarkable feature of the SHG microscopic mechanism is the fact, that for $\omega \tau \sim 1$ parameters $Q_{1}$ and $Q_{2}$ contain real and imaginary parts, moreover, the phases of these quantities are different. By that, excitation with linearly polarized radiation may cause circularly polarized light at a double frequency. Indeed, if the incident radiation contains both $x$ and $y$ components of $\boldsymbol{E}$, then the response at the double frequency also contains $j_{x}$ and $j_{y}$, however, their oscillations are phaseshifted. Thus, the second harmonic radiation becomes, in general, elliptically polarized. Calculation shows that the degree of circular polarization of the emission can reach $90 \%$ [106]. Note, that this effect is not observed so far.

It is worth to mention that the response of graphene at a double frequency due to the outlined mechanisms can be much stronger than in conventional semiconductor systems, since electron velocity in graphene exceeds Fermi velocity of electrons in semiconductor heterostructures. We compare the second order response in graphene with that of a twodimensional centrosymmetric system with parabolic energy spectrum $\left(Q_{1}^{\text {parab }}, Q_{2}^{\text {parab }}\right)$. In the high frequency limit $(\omega \tau \gg 1$, $\hbar \omega \ll E_{F}$ ) Eqs. (25) yield $Q_{2}=Q_{1} / 2$ and we obtain the enhancement factor [74]

$$
\eta=\frac{Q_{1}^{\text {graphene }}}{Q_{1}^{\text {parab }}}=\frac{v^{2}}{2 v_{F}^{2}}
$$

where $v=10^{8} \mathrm{~cm} / \mathrm{s}, v_{F}=\sqrt{2 E_{F} / m}$ is the Fermi velocity of electrons in the quantum well structure, $m$ is their effective 

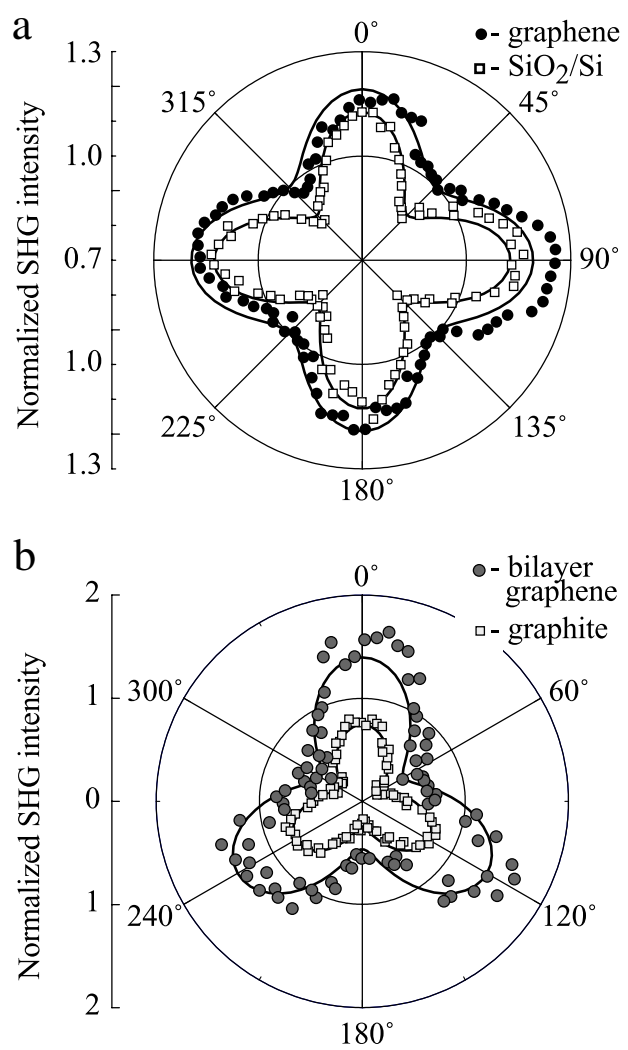

Fig. 5. (a) Polarization dependence of the normalized second harmonic radiation intensity detected from graphene on $\mathrm{SiO}_{2} / \mathrm{Si}_{\mathrm{i}}$ substrate (filled circles) and purely $\mathrm{SiO}_{2} / \mathrm{Si}$ substrate (open squares) measured for the fundamental harmonic wavelength $\lambda=800 \mathrm{~nm}$ at room temperature. Here the polar angle $\gamma$ is the angle between the incidence plane and [100]-axis of the substrate (see text for details). Solid curves are fits according to Eq. (27). (b) Normalized second harmonic radiation intensity detected from bilayer graphene on $\mathrm{SiO}_{2} / \mathrm{Si}$ (filled circles) and from bulk graphite (open squares) and a function of $\gamma$. Solid curves show the fits after Eq. (28) and are normalized such that the isotropic component of the second harmonic signal from silicon would be unity. The absolute angle is arbitrary for both curves. After [49].

mass. For typical Fermi velocities on the order of $v_{F}=2 \times 10^{7} \mathrm{~cm} / \mathrm{s}$ one has the enhancement factor

$$
\eta \sim 10
$$

Hence, the second harmonic response in graphene may be about an order of magnitude larger than that of other twodimensional semiconductor systems. Moreover, it can further be enhanced due to the excitation of plasmons as suggested in Ref. [74].

\subsubsection{Experiment}

Second harmonic generation has been first observed in single and multilayer graphene samples on $\mathrm{SiO}_{2} / \mathrm{Si} \mathrm{substrates}$ applying near-infrared radiation [49,50]. The experiments on harmonic generation reported so far applied linearly polarized pump beams and the linearly polarized response has been analyzed. The nonlinear optical effects sensitive to the radiation helicity of the pump beam or resulting in generation of a circularly polarized light are still a challenge. In agreement with the phenomenological theory presented in Section 3.4, second harmonic has been observed applying radiation at oblique incidence. Either $p$ - or $s$-polarized beam of a femtosecond Ti:Sapphire laser operating with pulse energy $\approx 0.06 \mathrm{~nJ}$ and duration about $150 \mathrm{fs}$ in the wavelength range of $730-830 \mathrm{~nm}$ has been used. This experimental situation where $\hbar \omega>E_{F}$ corresponds to the quantum frequency range. The radiation falls on a graphene layer at an angle of incidence of $\theta=60^{\circ}$ and is focused into an elliptical spot size of approximately 7-10 $\mu \mathrm{m}$. We note that in graphene, which is strictly two-dimensional system, the phase synchronization condition needed for harmonic generation in bulk materials is relaxed. The signal at a double frequency is collected, optically filtered from the fundamental light, and detected using a cooled photomultiplying tube and photon-counting electronics. It has been verified that the intensity of the second harmonic emission is proportional to the square of that for the incident radiation. The variations of the signal upon rotation of the radiation polarization vector as well as rotation the sample about the normal axis have been analyzed. Both methods allow the detailed characterization of the second harmonic and, together with averaging over many rotations, improve the signal/noise ratio. The latter is of importance, since the second harmonic intensity from a small graphene sheet is very weak-a few photons per second. While all four combinations of $s$ - and $p$-polarized fundamental and second harmonic light have been measured, the highest second harmonic intensity has been detected for $p$-polarization of both beams.

The evidence of the SHG in the single layer samples requires careful analysis of the data, in particular, of the dependence on the incidence plane orientation characterized by an angle $\gamma$ between the incidence plane and [100] axis of the substrate, see Fig. 5. As it follows from the phenomenological theory described above in Section 3.4, the second harmonic emission from 


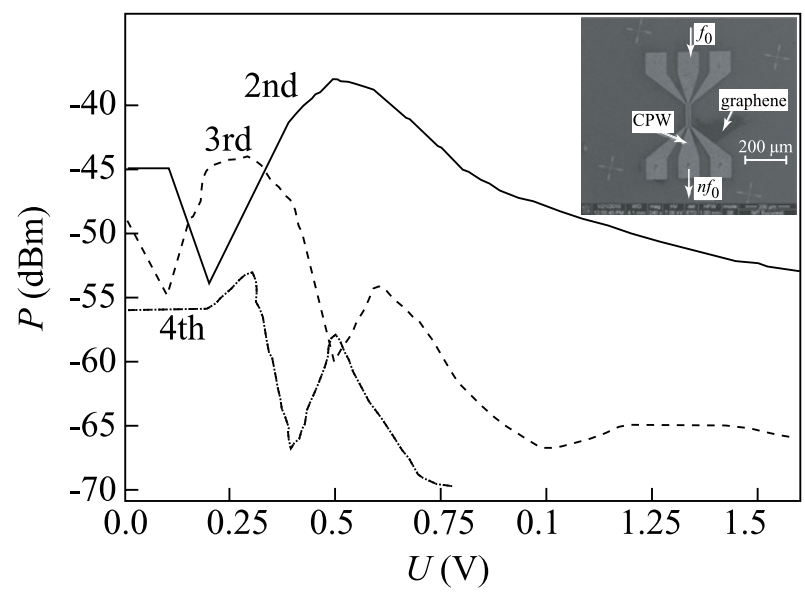

Fig. 6. Output power $P$ as a function of $d c$ bias voltage $U$ for the second-order (solid line), third-order (dashed line), and fourth order (dash-dotted line) harmonics excited by radiation with frequency of $f_{0}=1 \mathrm{GHz}$. Inset shows metallic coplanar line waveguide graphene multiplier device. After [48].

graphene monolayer is isotropic, its intensity should not change upon variation of the angle $\gamma$. However, experimentally two contributions, the isotropic ( $\gamma$-independent) and quadrupolar $(\alpha \cos 4 \gamma)$ contributions are observed in single layers. As a result, the normalized SHG intensity as a function of angle $\gamma$ can be described by the following fitting equation:

$$
I(\gamma)=A_{0}+A_{4} \cos (4 \gamma+\delta),
$$

where $A_{0}$ and $A_{4}$ are the amplitudes of the isotropic and quadrupolar components and $\delta \approx 0$ is the phase. The problem in analysis is that such a behavior is expected and indeed observed from the bare Si substrate [50,111]. However, contribution of graphene to the second harmonic manifests itself by reduction of anisotropy by about 30\% and an increase of intensity, compared with bare substrate. This result is in agreement with phenomenological description, Eqs. (16), which demonstrates that the graphene response is isotropic: The intensity of the second order response is the same irrespective of the orientation of the incidence plane.

The situation drastically changes in multilayer graphene. Here, instead of four-fold, the symmetry of photoresponse becomes three-fold, which rules out the substrate contribution. It is shown in Fig. 5, where the normalized second harmonic intensity is plotted as a function of $\gamma$. The experimental results now follow the phenomenological equation

$$
I(\gamma)=A_{0}^{\prime}+A_{3}^{\prime} \cos \left(3 \gamma+\delta^{\prime}\right)
$$

where $A_{0}^{\prime}$ and $A_{3}^{\prime}$ are the amplitudes of isotropic (zeroth) and third angular harmonics, $\delta^{\prime}$ is the initial phase. In this case the fourth angular harmonic is absent, indicating that the response is dominated by the multilayer graphene rather than by a substrate. The data not only demonstrate a pure multilayer graphene response but also indicate the symmetry reduction to $C_{3 \mathrm{v}}$ supporting the effect of the substrate induced structure inversion asymmetry, see Section 3.3. Based on this difference in Ref. [49] second harmonic generation effect was suggested for the diagnosing the layering structure of graphene samples. As recently shown in Ref. [51] the SHG can also be observed in flat graphene at the normal incidence, however, only if additionally an in-plane static field is applied to graphene sheet. These processes are already third order in electric field and will be discussed from the theoretical point of view in Section 6.

The discussed above experiments on the second harmonic generation apply infrared radiation with $\hbar \omega \gg E_{F}$ which corresponds to the quantum regime of light-matter interaction. Moreover, the second and higher harmonic generation have been observed also for the gigahertz frequency range (wavelengths of the order of several millimeters) where the classical frequency range was realized [48]. For measurements of the microwave frequency multiplication a specific high-frequency structure, i.e., a metallic coplanar line waveguide device, was patterned directly on graphene, see inset in Fig. 6 . Importantly, the current-voltage characteristic of used device is linear, ruling out possible mechanisms based on the nonlinear coupling between quasi-static field and current response. Thus, the physical mechanism of frequency multiplication is related with strongly nonlinear electromagnetic response of Dirac fermions in graphene. Fig. 6 shows the dependence of the signal at double frequency on the bias voltage, as well as the powers of the third and fourth harmonics measured on the same device. The second order nonlinear effect appears at zero bias its power varies from - 60 (at excitation with $10 \mathrm{GHz}$ ) to - 45 dBm (for $1 \mathrm{GHz}$ ) as compared to the power of excitation frequency. In contrast to the measurements under infrared excitation [51], here the static voltage does not lead to an enhancement of the signal. Rather strong nonlinear response suggests that such graphene-based systems can be efficiently implemented as frequency multipliers in $\mathrm{GHz}$ and may be even in $\mathrm{THz}$ ranges. Note that the latter is not yet realized.

\subsection{Dynamic hall (photon drag) effect}

\subsubsection{Microscopic theory}

The microscopic theory for the photon drag effect in graphene was developed in Refs. [58,59] for classical frequency range and in Ref. [62] for the quantum frequency range. Here we start with the presentation of the results of microscopic 
calculations for the classical range of frequencies, $\hbar \omega \ll E_{F}$, based on the Boltzmann equation approach and considering the classical picture of the effects visualized in the model outlined in Section 4. Amplitude and the sign of the resulting net $d c$ current $\boldsymbol{j}$ are given by the constants $T_{1}, T_{2}$, and $\tilde{T}_{1}$ in Eqs. (10). Calculations carried out in Refs. [58,59] and taking into account both $E B$ and $q E^{2}$ contributions yield

$$
\begin{aligned}
& T_{1}=-\frac{2 e^{3} v^{4}}{\omega} \sum_{\boldsymbol{k}} \frac{\tau_{1} f_{0}^{\prime}}{1+\omega^{2} \tau_{1}^{2}}\left[2\left(\frac{d \tau_{1}}{d \varepsilon_{k}}+\frac{\tau_{1}}{\varepsilon_{k}}\right)-\frac{1-\omega^{2} \tau_{1} \tau_{2}}{1+\omega^{2} \tau_{2}^{2}}\left(\frac{d \tau_{1}}{d \varepsilon_{k}}-\frac{\tau_{1}}{\varepsilon_{k}}\right)\right], \\
& T_{2}=-\frac{2 e^{3} v^{4}}{\omega} \sum_{\boldsymbol{k}} \frac{\tau_{1} f_{0}^{\prime}}{1+\omega^{2} \tau_{1}^{2}}\left(\frac{d \tau_{1}}{d \varepsilon_{k}}-\frac{\tau_{1}}{\varepsilon_{k}}\right), \\
& \tilde{T}_{1}=e^{3} v^{4} \sum_{\boldsymbol{k}} \frac{\tau_{1}^{2}\left(1+\tau_{2} / \tau_{1}\right) f_{0}^{\prime}}{\left[1+\left(\omega \tau_{1}\right)^{2}\right]\left[1+\left(\omega \tau_{2}\right)^{2}\right]}\left(\frac{d \tau_{1}}{d \varepsilon_{k}}-\frac{\tau_{1}}{\varepsilon_{k}}\right) .
\end{aligned}
$$

Eqs. (29) show that the radiation frequency is an important issue for the current generation. The frequency dependence of linear and circular currents given by $T_{1}$ and $\tilde{T}_{1}$, respectively, is shown in the inset in Fig. 9 together with experimental data discussed in detail later, in Section 5.2.3. In line with qualitative model shown in Fig. 4 in the limit of $\omega \rightarrow 0$ the linear photocurrent is constant while circular one is zero. With the frequency increasing, i.e., for $\omega \tau \gg 1$ but $\hbar \omega \ll E_{F}$, the linear photocurrent decreases as

$$
j_{B}, j_{C} \propto \frac{1}{\omega^{2}}
$$

Moreover, due to an interplay of $E B$ and $q E^{2}$ contributions the linear photocurrent in the incidence plane not only decreases but may change its sign as a function of the radiation frequency depending on the dominant scattering mechanism [59]. By contrast, the circular photocurrent exhibits nonmonotonic frequency dependence: It rises with increasing frequency, reaches the maximum magnitude at $\omega \tau \sim 1$ and then drops down as $\left(\hbar / \tau \ll \hbar \omega \ll E_{F}\right)$

$$
j_{A} \propto \frac{1}{\omega^{3} \tau}
$$

Although the drag effects are suppressed with an increase of frequency, they may still result in the observable signals, see below. Such frequency dependence is in agreement with the phenomenological considerations. Indeed, the time reversal symmetry imposes restrictions on the constants $T_{1}, T_{2}$, and $\tilde{T}_{1}$ in Eqs. (10) and, hence, on the parameters $A, B$, and $C$ in Eqs. (11) governing their frequency dependence. To illustrate these limitations, we consider the regime of low frequencies, $\hbar \omega \ll E_{F}$, where only intraband transitions are possible. We note that the following quantities: $\mathbf{j}, \mathbf{q}, \omega \tau$, and $P_{\text {circ }}$ are odd at the time reversal, while radiation intensity, $I$, is even at the time reversal. Phenomenological equations (2) are invariant at time reversal. It follows from Eq. (10) that linear photocurrent is given by

$$
j_{\alpha} \propto q_{\beta} \mathcal{F}_{d, l}(\omega \tau) I
$$

where $\widetilde{F}_{d, l}(\omega \tau)$ is a function, forced to be even at time reversal. Hence, $\mathscr{F}_{d, l}(\omega \tau)$ contains only even powers of $\omega \tau$. By contrast, the circular photon drag effect given by Eq. (10b)

$$
j_{\alpha} \propto q_{\beta} P_{\text {circ }} \mathcal{F}_{d, c}(\omega \tau) I
$$

is described by the function $\mathcal{F}_{d, c}(\omega \tau)$ odd at time reversal, hence, containing only odd powers of $\omega \tau$. Similar relations are satisfied for the photogalvanic effect given by Eqs. (12), in the latter case, however, since $\boldsymbol{q}$ does not enter the phenomenological expressions, function describing circular photon drag effect is even at time reversal, and vice versa.

With further increase of the radiation frequency or decrease of the Fermi energy we turn to the quantum frequency range. We present the results for the case of $\omega \tau \gg 1$ and $\hbar \omega \sim E_{F}$ studied in Ref. [62]. The absorption of the electromagnetic wave in the case of intraband transitions should be accompanied with the electron scattering, otherwise energy and momentum conservation laws cannot be satisfied. The corresponding processes are schematically depicted in Fig. 7. As a result, one can express the coefficients $T_{1}$ and $T_{2}$ describing linear photocurrent in the following form $(\omega \tau \gg 1)[62]$

$$
\begin{aligned}
& T_{1}=-e^{3} v^{4} \frac{32}{\hbar \omega^{4}} \sum_{\boldsymbol{k}_{i}}\left[f\left(\varepsilon_{k_{i}}\right)-f\left(\varepsilon_{k_{f}}\right)\right] \frac{\varepsilon_{k_{f}}}{\left(\varepsilon_{k_{i}}+\varepsilon_{k_{f}}\right)^{2}}, \\
& T_{2}=-e^{3} v^{4} \frac{8}{\hbar \omega^{4}} \sum_{\boldsymbol{k}_{i}}\left[f\left(\varepsilon_{k_{i}}\right)-f\left(\varepsilon_{k_{f}}\right)\right] \frac{\varepsilon_{k_{f}}^{2}+\varepsilon_{k_{i}}^{2}+(\hbar \omega)^{2}}{\varepsilon_{k_{i}}\left(\varepsilon_{k_{i}}+\varepsilon_{k_{f}}\right)^{2}} .
\end{aligned}
$$

Here $\varepsilon_{k_{f}}=\varepsilon_{k_{i}}+\hbar \omega$. It is noteworthy that Eqs. (32) are valid provided $\hbar \omega<E_{F}$. We note that although the scattering rates are not explicitly present in Eqs. (32), the scattering processes are crucial for the photocurrent formation. 
a

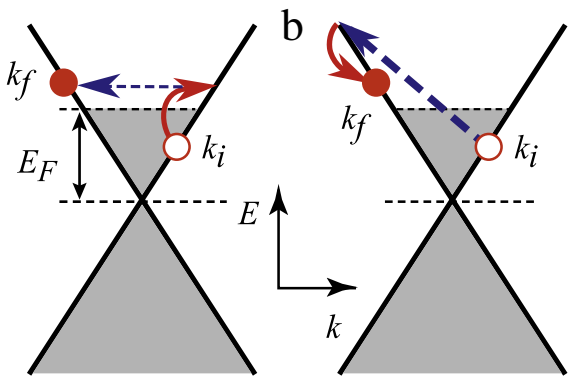

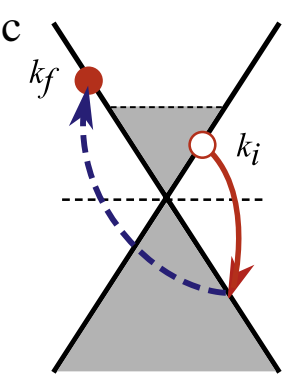

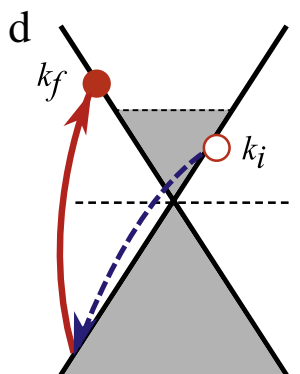

Fig. 7. Schematic illustration of the processes responsible for the photon drag effect in the quantum frequency range under intraband transitions $(\omega \tau) 1$, $\left.\hbar \omega \leqslant E_{F}\right)$. Panels (a)-(d) show phonon/impurity assisted indirect optical transitions via different intermediate virtual states. Arrows show electron-photon interaction (solid arrows) and electron scattering caused by phonons or impurities (dashed arrows). Initial and final states of a photoexcited carrier with wavevectors $k_{i}$ and $k_{f}$ are shown by open and solid circles, respectively.

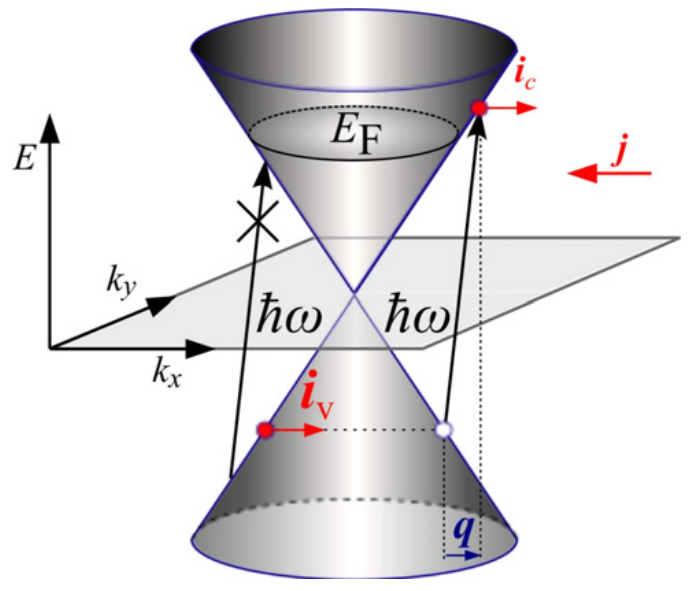

Fig. 8. Schematic illustration of the resonant photon drag effect under interband optical transitions. The tilted arrows show optical interband transitions inclined in the $\boldsymbol{k}$ space due to the transfer of photon momentum $\boldsymbol{q}$ to electrons. At resonance the final state of the transitions with negative $k_{x}$ lies below the Fermi energy and, therefore, such transitions are forbidden. By contrast the transitions with positive $k_{x}$ are allowed. The optically induced imbalance of carriers in the $\boldsymbol{k}$-space results in fluxes of electrons (filled circuits) in the conduction, $\boldsymbol{i}_{c}$, and valence, $\boldsymbol{i}_{v}$ bands. Both fluxes contribute constructively to the electric current, $\boldsymbol{j}=e\left(\boldsymbol{i}_{c}+\boldsymbol{i}_{v}\right)$. After [60].

Note, that if the photon energy becomes much smaller as compared with the electron energies, $\hbar \omega \ll \varepsilon_{k_{i}}, \varepsilon_{k_{f}}$, but $\omega \tau_{1}, \omega \tau_{2} \gg 1$, the classical and quantum approaches merge. One can check that, in agreement with Eqs. (30), Eqs. (32) yield

$$
T_{1}=2 T_{2}=\frac{8 e^{3} v^{4}}{\omega^{3}} \sum_{k} \frac{f_{0}^{\prime}}{\varepsilon_{k}} .
$$

In this frequency range values of $T_{1}$ and $T_{2}$ are identical to those presented in Eqs. (29).

\subsubsection{Resonant drag effect under interband transitions}

A further increase of the radiation frequency or decrease of the Fermi energy opens another absorption channel, namely, if $\hbar \omega \geqslant 2 E_{F}$ the direct interband transitions dominate the absorption of radiation. It gives rise to the novel regimes of the photon drag effect as it is considered theoretically in Ref. [60]. Schematics of the photocurrent generation is illustrated in Fig. 8. The microscopic origin of the photocurrent generation in this quantum frequency range is related with the fact that the electron in the process of transition from the valence band to conduction band shifts in the $\boldsymbol{k}$ space by $\boldsymbol{q}$, the photon wavevector.

In the narrow frequency range

$$
\left|\hbar \omega-2 E_{F}\right| \leqslant \hbar v q \text {, }
$$

as it is seen from Fig. 8, only transitions at positive momenta are possible due to the final state filling effect. It results in the strong asymmetry of photoelectrons distribution, which gives rise to the resonant photocurrent. The interband absorption gives rise to the generation of electron-hole pairs. As a result, a photocurrent is contributed both by the photoelectrons and photoholes. The hole contribution can be viewed as that of a valence band electron with an opposite wavevector. These fluxes of conduction and valence band electrons are shown by arrows in Fig. 8. Since the velocity of quasiparticle is given by $\hbar^{-1} \mathrm{~d} \varepsilon / \mathrm{d} \boldsymbol{k}$, the velocities for opposite wavevectors in the conduction and valence band are the same. Consequently, the 


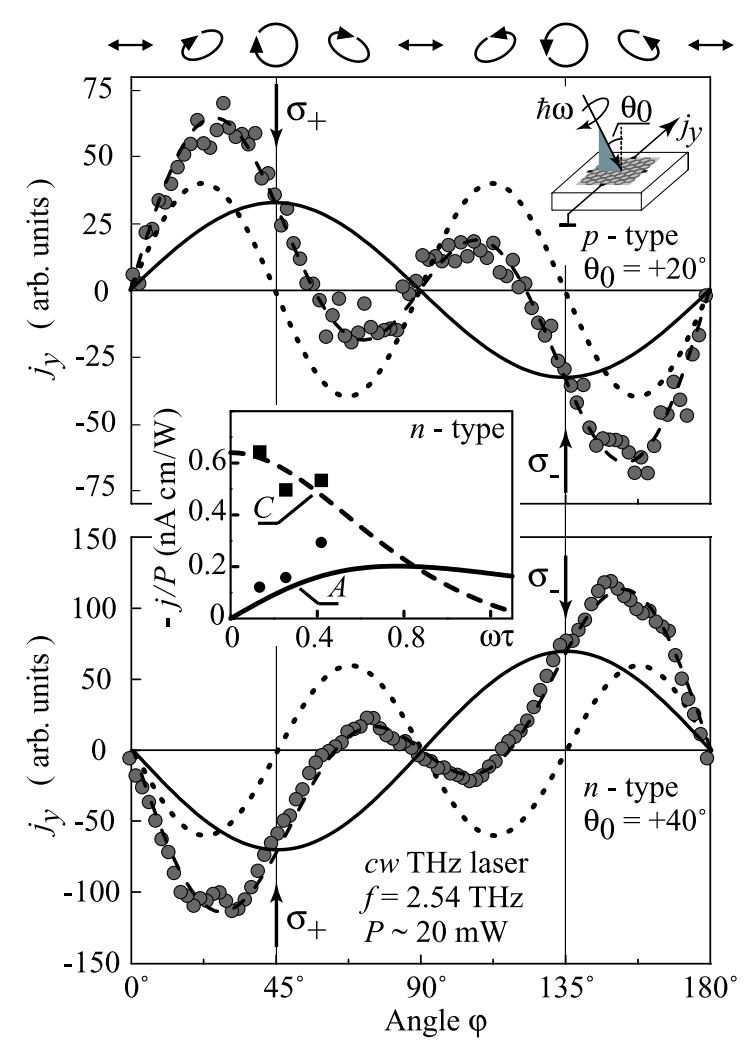

Fig. 9. Helicity dependence of the photocurrent, $j_{y}$, measured in the direction normal to the plane of incidence. The ellipses on top illustrate the polarization states for various angles $\varphi$. Dashed lines show fits to the calculated total current $j_{A}+j_{B}$ comprising the circular contribution $j_{A}$ (full line) and the linear contribution $j_{B}$ (dotted line), see Eq. (11a). Top and bottom panels correspond to $p$ - and $n$-type samples, respectively and demonstrate that the current has opposite signs for opposite carrier polarity. Inset shows frequency dependence of the longitudinal linear, $j_{C}$, and circular, $j_{A}$, photocurrents. Circles and squares are experimental data, solid and dashed curves represent the results of calculation. The agreement is obtained without fitting parameters. After [58].

fluxes in the conduction and valence bands are the same. Taking into account that the electron generation rate is $\pi \alpha I /(\hbar \omega)$, where $\pi \alpha$ is the monolayer graphene absorbance ( $\alpha$ is the fine structure constant) [23,112], and all generated electrons contribute with velocity $v$ to the drag current one has

$$
j \sim \operatorname{ev} \tau \pi \alpha \frac{I}{\hbar \omega} .
$$

This effect, known as resonant drag effect, was suggested in Ref. [60]. Although the magnitude of the current is independent of the photon wavevector $\boldsymbol{q}$, the resonant effect takes place in the narrow frequency range, Eq. (34), the smaller the smaller $q$. If the photon frequency is high enough, $\hbar \omega-2 E_{F}>\hbar v q$, the resonant contribution is absent and the ordinary (nonresonant) drag current is formed, similarly to the case of semiconductor quantum well structures [113].

\subsubsection{Experiment}

Dynamic Hall and photon drag effects have been demonstrated applying $\mathrm{THz}$ and infrared laser radiation to unbiased graphene layers produced both by exfoliation and epitaxial techniques $[58,59] .{ }^{8}$ In all experiments known so far, the limit $\hbar \omega<E_{F}$ was fulfilled, and, therefore, classical regime of light-matter interaction is realized. To prevent high losses or electrical shunting by conducting substrates high-resistivity Si or semi-insulating SiC substrates have been used. For some samples nonconductive polymer films were used for protection of graphene samples from the undesired doping in the ambient atmosphere [114,115]. To measure photocurrents ohmic contacts were made at samples edges. Details on the material growth and characterization can be found in [58,115-117]. For optical excitation $c w$ and pulsed molecular optically pumped terahertz lasers (for laser characteristics see e.g. [118-122]) or tunable $\mathrm{CO}_{2}$ lasers were applied. In the measurements the spatial beam distribution had an almost Gaussian profile, independently measured by a pyroelectric camera [123], and the laser spot is always centered between the contacts. This arrangement prevents the temperature gradient between contacts necessary for the thermoelectric effect like that discussed for graphene in e.g. [124]. A pronounced signal is detected in a wide range of radiation frequencies, from $0.6 \mathrm{THz}(\lambda \approx 500 \mu \mathrm{m})$ up to about $30 \mathrm{THz}(\lambda=10 \mu \mathrm{m})$,

\footnotetext{
8 While both types of samples showed the effect, the micrometer sized exfoliated samples displayed an additional edge contribution (discussed below in Section 5.4) as the spot size of the terahertz laser of $1 \mathrm{~mm}^{2}$ was larger than the graphene flakes.
} 
a

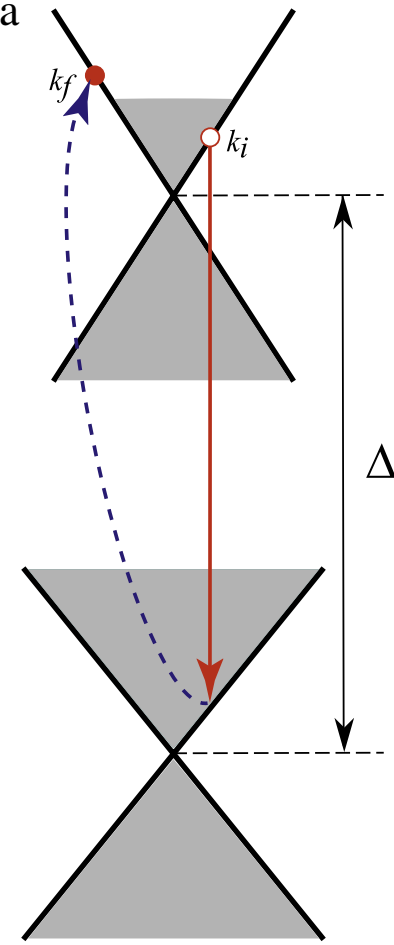

b
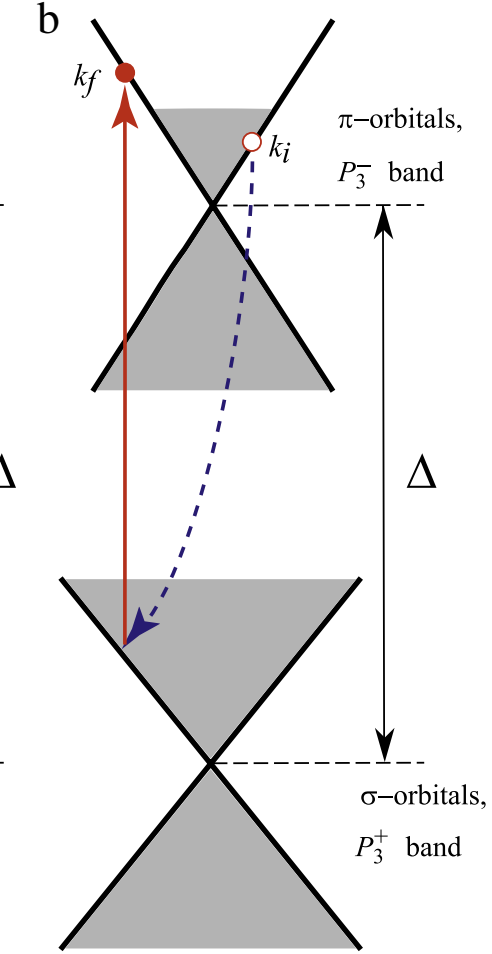

Fig. 10. Schematic illustration of indirect intraband Drude transitions with intermediate states in $P_{3}^{+}$band. These transitions together with those shown in Fig. 7 are important for photogalvanic effect, see text for details. After [62].

and intensities, from $\mathrm{mW} / \mathrm{cm}^{2}$ up to $\mathrm{MW} / \mathrm{cm}^{2}$. In agreement with theory presented above, the Hall photocurrent appears under oblique incidence. Fig. 9 shows results obtained on epitaxial single layer graphene excited by elliptically polarized light in transverse geometry. The polarization state of light was controlled by the rotation of the quarter-wave plate. This figure reveals that the photocurrent signal is a superposition of circular and linear contributions of comparable strengths. We emphasize that the circular contribution $\left(\boldsymbol{j} \propto P_{\text {circ }}=\sin 2 \varphi\right)$ manifests itself as a change of current direction for left and right circularly polarized radiation. In accordance with the theory, Section 3.1, the circular photocurrent is observed in the direction perpendicular to the incidence plane, while linear contribution is detected in the incidence plane together with polarization independent current. Obviously, the latter effects can be and indeed have been observed for linearly polarized radiation. Functional behavior of the photocurrent components upon variation of the radiation polarization state, incidence angle and frequency is in a full agreement with that obtained theoretically in Section 5.2.1. Moreover, the microscopic theory yields the absolute value of the photocurrent without fitting parameters with only assumption of the short-range scattering [58]. It is worth to note, that in agreement with theoretical consideration the signal reverse its sign by change of carrier type from $p$ to $n$. Strikingly, due to the fact that the conduction- and valence-band are symmetric with respect to the Dirac point, the opposite polarities of the signal can be observed in the same sample just by changing the Fermi level position.

\subsection{Photogalvanic effect in the pristine graphene}

\subsubsection{Microscopic theory}

Due to symmetry arguments addressed above, photogalvanic effect may emerge only in graphene systems where the inversion symmetry is broken. Moreover, from the same arguments summarized in Eqs. (12a), (12b) it follows that the photocurrent in flat infinite graphene can be generated only with allowance for $z$-component of the incident electric field. The latter condition hampers the photogalvanic effect formation. Indeed, for strictly two-dimensional model where only $\pi$-orbitals of carbon atoms are taken into account, no response at $E_{z}$ is possible. However, taking into account other bands in electron energy spectrum formed from the $\sigma$-orbitals of carbon atoms gives rise to the $d c$ current.

Microscopically, the photogalvanic effect arises due to the quantum interference of the Drude-like indirect optical transitions represented in Fig. 7 (for $\boldsymbol{q}=0$ ) and the indirect intraband transitions with intermediate states in distant bands depicted in Fig. 10, similarly to the orbital mechanisms of the photogalvanic effects in conventional semiconductor nanostructures [125-127]. To illustrate the generation of the photocurrent we consider the circular photogalvanic effect. The current results from the anisotropic distribution of photoexcited electrons, which is caused the different dependence of the transition matrix elements on the wavevectors: The matrix element of the Drude-like transitions within one band (Fig. 7) is linear in the wavevectors

$$
M_{\boldsymbol{k}_{f}, \boldsymbol{k}_{i}}^{(1)} \propto \boldsymbol{A} \cdot\left(\boldsymbol{k}_{i}-\boldsymbol{k}_{f}\right),
$$


whereas the matrix element of the indirect optical transitions involving distant bands $M_{\boldsymbol{k}_{f}, \boldsymbol{k}_{i}}^{(2)}$ (Fig. 10) is almost $\boldsymbol{k}_{i}$ and $\boldsymbol{k}_{f}$ independent. The total transition rate calculated with allowance for the quantum mechanical interference is given by

$$
W_{\boldsymbol{k}_{f}, \boldsymbol{k}_{i}} \propto\left|M_{\boldsymbol{k}_{f}, \boldsymbol{k}_{i}}^{(1)}+M_{\boldsymbol{k}_{f}, \boldsymbol{k}_{i}}^{(2)}\right|^{2} .
$$

As a result we obtain from Eq. (36) that besides $\boldsymbol{k}$-even contributions $\left(\propto\left|M_{\boldsymbol{k}_{f}, \boldsymbol{k}_{i}}^{(1)}\right|^{2},\left|M_{\boldsymbol{k}_{f}, \boldsymbol{k}_{i}}^{(2)}\right|^{2}\right)$, the transition probability contains $\boldsymbol{k}$-odd interference term:

$$
\propto 2 \operatorname{Re}\left[M_{\boldsymbol{k}_{f}, \boldsymbol{k}_{i}}^{(1)} M_{\boldsymbol{k}_{f}, \boldsymbol{k}_{i}}^{(2) *}\right] \propto \boldsymbol{A} \cdot\left(\boldsymbol{k}_{i}-\boldsymbol{k}_{f}\right) .
$$

It follows from Eq. (37) that the interference contribution is linear in the initial and final wavevectors, $\boldsymbol{k}_{i}$ and $\boldsymbol{k}_{f}$, hence, the distribution function of the photoexcited carriers is anisotropic in the $\boldsymbol{k}$-space. An imbalance of electron population in different regions of the $\boldsymbol{k}$-space results in the $d c$ current. We stress that the matrix element $M^{(1)}$ contains the in-plane components of the radiation vector potential, while the element $M^{(2)}$ is related with its $z$-component. Hence, the generated current is proportional to $\boldsymbol{E}_{\|} E_{z}^{*} \pm$ c.c. in accordance with phenomenological analysis, see Eq. (12).

As it follows from the above consideration, the optical transitions via distant bands, although providing a tiny fraction in the total absorption of graphene, are crucial for the current formation. Therefore, we consider them in more detail. Here, the distant bands, involved in the interference, are described by $P_{3}^{+}$representation (even under the $z \rightarrow-z$ reflection), while the conduction and valence band states in graphene transform according to the $P_{3}^{-}$representation (odd under the $z \rightarrow-z$ reflection) [78]. Microscopic calculations performed within the basis of $2 s$ and $2 p$ atomic orbitals [78,79] show that the distance from the $P_{3}^{-}$states forming conduction and valence bands and closest deep valence bands $P_{3}^{+}, \Delta$, is about $10 \mathrm{eV}$. It is noteworthy, that the electron dispersion in these bands has the form, similar to that of conduction and valence bands: i.e. energy spectrum near $K$ (or $K^{\prime}$ ) point is linear, however, with different velocity, as it is schematically illustrated in Fig. 10. Since matrix elements $M^{(1)}$ and $M^{(2)}$ have different parity under $z \rightarrow-z$ reflection, the quantum interference is only possible in the case, where the graphene is deposited on the substrate and/or adatoms are present on one side of the sample, i.e. where the $z \rightarrow-z$ reflection symmetry is broken.

In the further description we limit our consideration to the circular electric current only, $\boldsymbol{j} \propto \chi_{c} P_{\text {circ }}$, see Eqs. (12). The calculations carried out in framework of the Fermi golden rule for the quantum frequency range where $\omega \tau \gg 1$ and $\hbar \omega \lesssim E_{F}$ yield [62]:

$$
\chi_{c}=-e v \frac{4 \pi w}{\hbar} \sum_{k_{i} k_{f}} \frac{\tau_{1}\left(\varepsilon_{k_{f}}\right) \varepsilon_{k_{i}}+\tau_{1}\left(\varepsilon_{k_{i}}\right) \varepsilon_{k_{f}}}{\varepsilon_{k_{i}}+\varepsilon_{k_{f}}}\left[f\left(\varepsilon_{k_{i}}\right)-f\left(\varepsilon_{k_{f}}\right)\right] \delta\left(\varepsilon_{k_{f}}-\varepsilon_{k_{i}}-\hbar \omega\right)
$$

where

$$
w=\frac{2 \pi e^{2} v p_{0}}{m_{0} c \omega^{2}} \frac{\left\langle V_{0} V_{1}\right\rangle}{\Delta^{2}},
$$

$V_{0}$ and $V_{1}$ determine the electron-impurity or electron-phonon scattering matrix elements within $\pi$-band and between $\sigma$ - and $\pi$-bands, respectively, $p_{0}$ is the interband optical matrix element, and $\left\langle V_{0} V_{1}\right\rangle$ denotes the averaging of the product $V_{0} V_{1}$ over the disorder realizations. The treatment of the general case is given in Ref. [62].

The direction of the current is determined by the sign of the product $\left\langle V_{0} V_{1}\right\rangle$ and the radiation helicity. The averaged product $\left\langle V_{0} V_{1}\right\rangle$ has different signs for the same impurities, but positioned on top or bottom of graphene sheet. It is clearly seen that the photogalvanic current vanishes in symmetric graphene-based structures where $\left\langle V_{0} V_{1}\right\rangle=0$.

In the case of the degenerate electron gas with the Fermi energy $E_{F}$ and in the limit of $\hbar \omega \ll E_{F}$ Eq. (38) can be recast as [62]

$$
\chi_{c}=-8 \frac{\alpha e d_{0}}{\Delta} \frac{\left\langle V_{0} V_{1}\right\rangle}{\left\langle V_{0}^{2}\right\rangle} \frac{E_{F}}{\hbar \omega},
$$

where we introduced effective dipole of interband transition

$$
e d_{0}=\frac{e p_{0} \hbar}{m_{0} \Delta} .
$$

Eq. (39) allows us to evaluate the frequency dependence of the circular photogalvanic effect. Namely, at $\omega \tau \gg 1, \hbar \omega \ll E_{F}$, the circular photocurrent behaves as $1 / \omega$, i.e. it is parametrically larger than the circular drag (or circular ac Hall) effect, which behaves as $1 / \omega^{3}$, see Eq. (31). This important property is related with the time reversal symmetry: the coefficient $\chi_{c}$ describing photogalvanic effect is even at time reversal, while $\tilde{T}_{1}$ describing circular drag effect is odd. Therefore, circular photocurrent formation due to photogalvanic effect is possible at the moment of carriers photogeneration. Since at $\omega \tau \gg 1$ for intraband transitions the absorption rate is proportional to the electron scattering rate, $\tau^{-1}$, and current density is proportional to the electron scattering time, $\tau$, the circular photocurrent is independent of the scattering rate. Owing to different symmetry under time reversal the linear photogalvanic effect, by contrast, requires extra scattering, its description within the same model is presented for the classical frequency range in Ref. [62]. As a result for $\omega \tau \gg 1$ (but $\hbar \omega<E_{F}$ ) the following hierarchy of the current magnitudes is possible: (i) circular photogalvanic effect $\propto \omega^{-1}$, (ii) linear photogalvanic $\propto \omega^{-2} \tau^{-1}$ and photon drag effects $\propto \omega^{-2}$, (iii) circular photon drag effect $\propto \omega^{-3} \tau^{-1}$. 


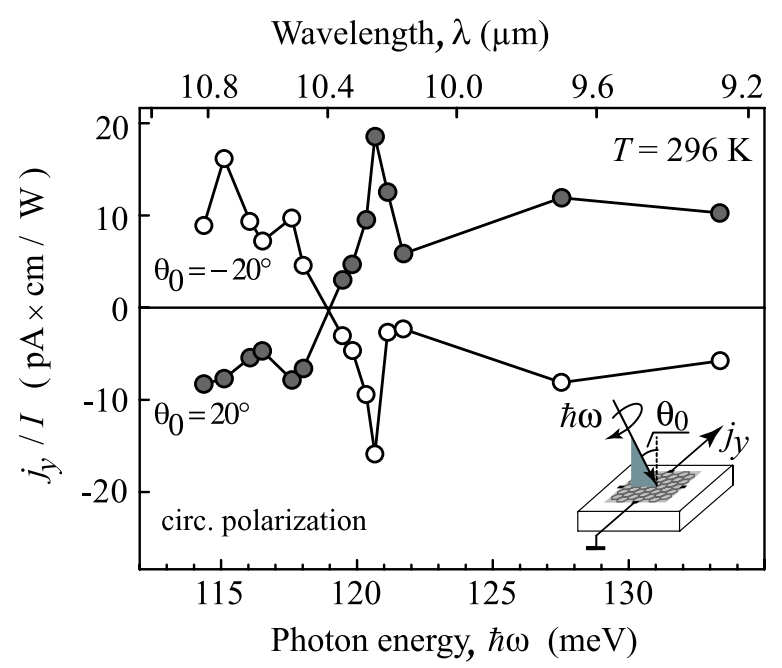

Fig. 11. Spectral dependence of the circular photocurrent $j_{y, A}$ measured in the direction normal to the plane of incidence. The data for epitaxial single layer graphene sample are obtained for circularly polarized infrared light $\left(\varphi=45^{\circ}\right)$ and two angles of incidence $\theta_{0}= \pm 20^{\circ}$. The inset shows the experimental geometry. After [62].

\subsubsection{Experiment}

As we emphasized above, see Section 3.2, the photogalvanic effects in pristine graphene involve $z$-component of electric field and may be observed only under special conditions, where the photon drag contribution is suppressed, in particular, in the quantum frequency range. Correspondingly, both the circular and linear photogalvanic effects were observed in the midinfrared range of radiation frequencies (about $30 \mathrm{THz}$ ) on epitaxial graphene samples. The demonstration of photogalvanic effects becomes possible due to two facts: On one hand, at such a high frequencies the photon drag effect is suppressed, and on the other hand, photogalvanic and drag effects appear to contribute to photocurrent with opposite signs. This interplay resulted in a change of sign of the photocurrent upon the variation of radiation frequency, see Fig. 11, providing an evidence for the existence and substantial contribution of the photogalvanic effect [62]. The value of the circular photocurrent caused by the photogalvanic effect is close to the theoretical estimate after Eq. (39) for sufficiently strong asymmetry degree, $\left\langle V_{0} V_{1}\right\rangle /\left\langle V_{0}^{2}\right\rangle \approx 0.5$. We emphasize that the photogalvanic effect does exist only due to the structure inversion asymmetry. Therefore, no photogalvanic effect is expected in graphene with equivalent "up" and "down" surfaces, e.g. in free standing graphene. It would be observable in such layers only for nonequal numbers of adatoms on the opposite sides of the graphene sheet. An experimental evidence for a large structure inversion asymmetry due to adatoms and/or substrate has been given most recently by observation and study of magnetic quantum ratchet effect in similar epitaxial samples [114].

\subsection{Edge photocurrents}

\subsubsection{Microscopic theory}

According to the symmetry analysis given in Section 3.2, the presence of sample edges breaks inversion symmetry of the system and, therefore, their illumination gives rise to the edge photocurrents, considered phenomenologically in Section 3.2. A microscopic process actuating the edge photocurrent generation is illustrated in Fig. 12(a) for the linearly polarized radiation where the semiinfinite sample occupying $x>0$ plane is shown together with the electron motion in the vicinity of the edge. The current formation involves the time dependent motion of the charge carriers under the action of the electric field and the diffusive scattering at the sample edge. The electric current is formed in the narrow stripe with the width on the order of the mean free path $\ell$ in the vicinity of the sample edge. It is contributed by the carries pushed towards the edge by the electric field in one half of a period, since for the diffusive scattering the electrons moving from the edge have random velocities along the boundary. We note that this mechanism is similar to that of the surface photogalvanic effect observed in bulk materials [128-131]. The above process results in the linear photogalvanic effect, given by the first term in phenomenological Eq. (14), $j_{y} \propto E_{x} E_{y}^{*}+E_{y} E_{x}^{*}$. The allowance for the trajectory winding under the action of circularly polarized radiation, shown in Fig. 12(b), results in the contribution to the current sensitive to the radiation helicity reversing sign from $\sigma^{+}$(solid) to $\sigma^{-}$(dashed). We note that the illumination of opposite edges of the sample results in the opposite sign of photocurrent (in a fixed frame of coordinates).

Edge photogalvanic effect may also result from the variation of the electron density in the vicinity of the edge due to the action of the field component perpendicular to the sample edge. To estimate the effect for the classical frequency range, $\hbar \omega \ll E_{F}$, we use the continuity equation

$$
\frac{\partial \delta N}{\partial t}+\frac{\partial i_{x}}{\partial x}=0
$$

which relates the variation of electron density $\delta N \equiv \delta N(x, t)=N(x, t)-N_{0}$ with the electron flux density $\boldsymbol{i}=\boldsymbol{j} / e$, where $N_{0}$ is the unperturbed electron density, and the coordinate frame with axis $y$ parallel to the edge is used (see Section 3.2). 

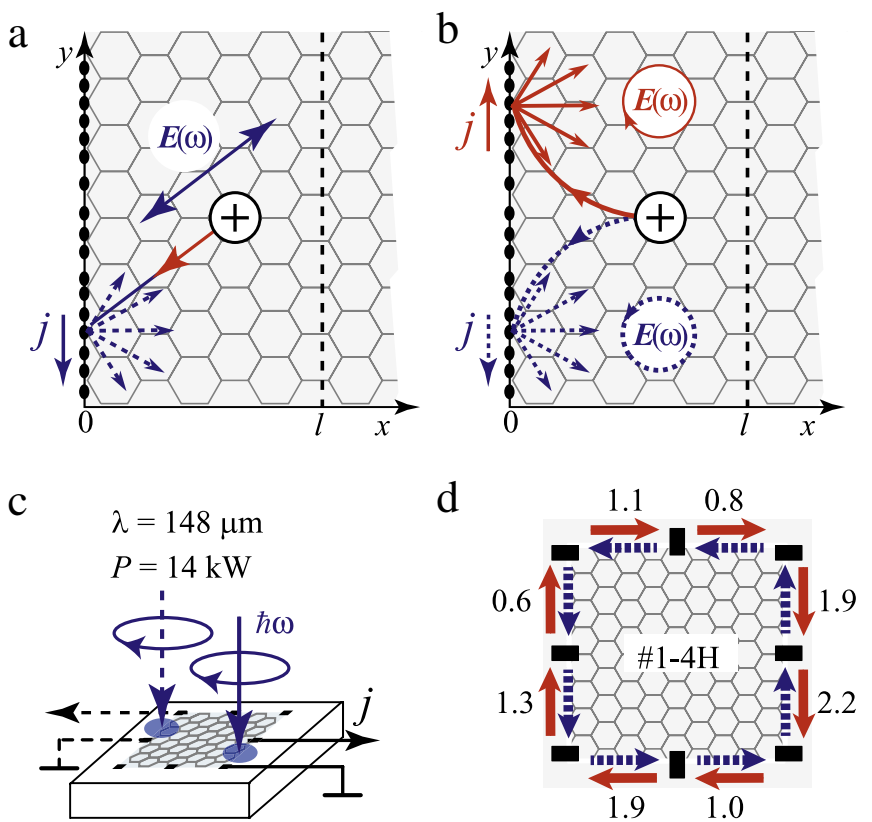

Fig. 12. (a) Schematic illustration of the linear edge photogalvanic effect. The oscillations of electric field $\boldsymbol{E}(\omega)$ are shown by double-sided arrow. The motion of a charge carrier (we consider holes for simplicity) towards the edge is shown by the solid red arrow, while the motion from the edge (after the diffusive scattering) is shown by multiple dashed arrows. The current is formed in the narrow stripe with the width of the mean free path $\ell$ near the edge. (b) Schematic illustration of the helicity driven edge current generation. The electric field of circularly polarized radiation rotates clockwise or counterclockwise resulting in a circular motion of carriers, which is sketched by solid and dashed curved trajectories, respectively. (c) Experimental geometry for the study of the edge photocurrents. (d) Edge photocurrent topology. Solid and dashed arrows show the current direction for $\sigma^{+}$and $\sigma^{-}$ polarizations, respectively. Numbers indicate the photocurrent amplitude $J_{A}$ in microamperes. After [61].

The $x$ component of the flux contains diffusive and drift contributions

$$
i_{x}=-D \frac{\partial \delta N}{\partial x}+\frac{\sigma(\omega)}{e} E_{x}
$$

where $\sigma(\omega)=C\left(N_{0}\right) \tau /(1-\mathrm{i} \omega \tau)$ is the frequency-dependent conductivity, $\tau$ is the momentum relaxation time, and $C\left(N_{0}\right)=e^{2} E_{F} / \pi \hbar^{2}[25,132]$. The electron gas is assumed to be degenerate, $E_{F}=\hbar v \sqrt{\pi N_{0}}$. The boundary conditions are as follows: at the sample edge $i_{x}=0$, while in the bulk of the sample the current is driven by the electric field only. As a result we have

$$
\delta N(x)=\delta N_{0} \exp \left(-\frac{1-\mathrm{i}}{l_{\text {eff }}} x\right),
$$

where $l_{\text {eff }}=\sqrt{2 D / \omega}=\ell / \sqrt{\omega \tau}, \ell=v \tau$ is the mean free path, $\delta N_{0}=\sigma(\omega) E_{x} l_{\text {eff }} /[e D(\mathrm{i}-1)]$. This description holds for $l_{\text {eff }} \gg \ell$. The electron density variation in the vicinity of the boundary is given by

$$
\Delta N=\int_{0}^{\infty} \delta N d x=\frac{\sigma(\omega) E_{x}}{\mathrm{i} \omega e} \equiv \delta N_{0} l_{\mathrm{eff}} /(1-\mathrm{i}) .
$$

The $d c$ edge photocurrent can be recast as a linear response to the $y$ component of electric field found with allowance for the $\Delta N$, the change of electron density induced by $E_{x}$ field component. The resulting expression for the total current $J_{y}=\int_{0}^{\infty} j_{y}(x) d x$ reads

$$
J_{y}=2 \operatorname{Re}\left\{\frac{\partial \sigma(0)}{\partial N_{0}} \Delta N E_{y}^{*}\right\}=\frac{\tau^{2}}{e} \frac{\mathrm{d} C^{2}\left(N_{0}\right)}{\mathrm{d} N_{0}} \operatorname{Re}\left\{\frac{E_{x} E_{y}^{*}}{\mathrm{i} \omega(1-\mathrm{i} \omega \tau)}\right\},
$$

contains both linear and circular components of the photocurrent in agreement with phenomenological expression (14). The divergence of circular photocurrent present in Eq. (44) for low frequencies, $\omega \tau \rightarrow 0$, results from the divergence of $l_{\text {eff }} \propto(\omega \tau)^{-1 / 2}$, and may be removed taking into account the self-consistent field, finite size of the illuminated area and finite size of the contacts used to measure the photocurrent. We note that edge photocurrents have also been treated in the framework of Boltzmann Eq. (24) in Ref. [61].

\subsubsection{Experiment}

The photon drag and photogalvanic effects, described in Sections 5.2 and 5.3 are induced in the "bulk" graphene layers applying $\mathrm{THz} / \mathrm{IR}$ radiation at oblique incidence and vanish for normal incidence. By contrast, edge photocurrents require the illumination of sample borders and have a maximum at the normal incidence of radiation. Experiments on edge 


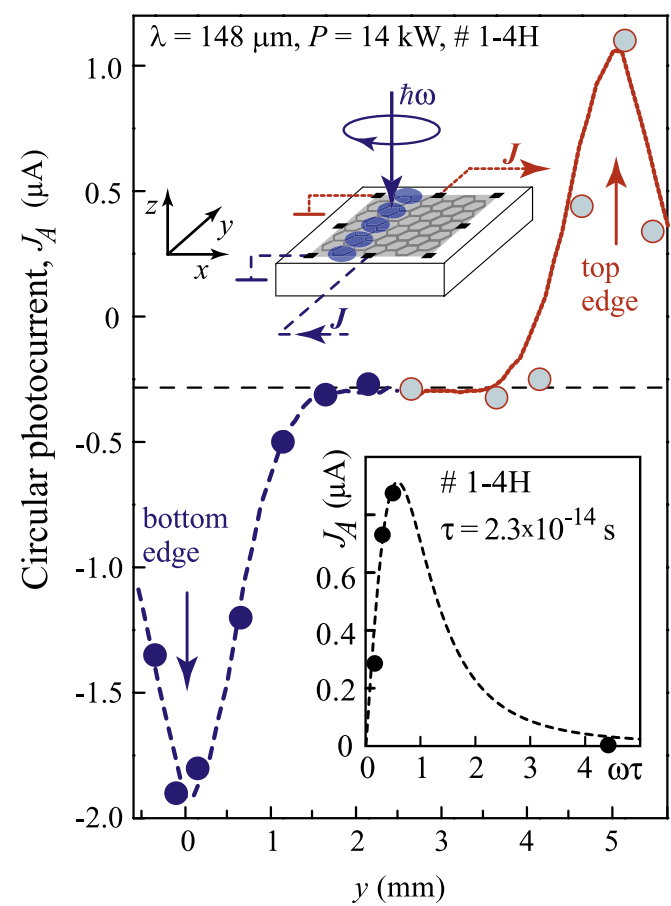

Fig. 13. Circular photocurrent $J_{A}$ measured in epitaxial single layer graphene sample as a function of the laser spot position. The laser spot is scanned along $y$ and the current is picked up from two contact pairs at the top (open circles) or bottom (full circles) sample edges aligned along $x$ (see inset). Lines represent the laser beam spatial distribution, which is measured by a pyroelectric camera and scaled to the current maximum. Top inset shows the scanning geometry. Bottom inset shows the measured circular photocurrent $J_{A}(\omega \tau)$ at one of the edge segments of sample (open circles) together with the fit after microscopic theory (dashed line) developed in the framework of the Boltzmann kinetic equation [61]. After [61].

photocurrents are challenging due to other types of photoresponses which may appear due to inhomogeneities, temperature gradients or illumination of contacts. However, this difficulty may be avoided by reduction of data analysis to the helicity dependent contribution, which changes its direction by switching the light polarization from right- to left-handed. Indeed, all effects mentioned above are unlikely to be sensitive to the direction of electric field rotation. While photocurrents have been observed in both large-area and small-area samples [61], the analysis of the edge photocurrents is much easier in the large-area samples. Indeed, in micrometer-sized exfoliated samples the radiation spot size is much larger than the graphene flakes and the effects of different edges are superimposed complicating the separation of edge contributions from the data. By contrast, in large area epitaxial samples, the illumination of a single edge by $\mathrm{THz}$ radiation could be realized enabling the accurate analysis of the individual edge currents.

Fig. 13 shows the circular edge photocurrent $J_{A}$ excited by THz radiation (classical range of frequencies) for the laser spot scanned across the large-area epitaxial graphene sample along the $y$-axis. The signal is picked up from a pair of contacts at the sample top and bottom edges oriented along the $x$-axis. The current reaches its maximum for the laser spot centered at the edge and rapidly decays with the spot moving. Comparison of the photocurrent with the laser spot cross-section (solid and dashed lines) shows that the signal just follows the Gaussian intensity profile showing that the current is due to illuminating the sample edges. The current direction for $\sigma^{+}$and $\sigma^{-}$circularly polarized radiation and the magnitude of $J_{A}$ for various contact pairs are shown in Fig. 12(d). In these measurements the Gaussian laser spot is always centered between the contacts preventing the temperature gradient between the contacts, at which signal is picked-up. The figure documents a remarkable behavior of the circular edge photocurrent: It forms a vortex winding around the edges of the square shaped samples, which reverses its direction upon switching from $\sigma^{+}$to $\sigma^{-}$light. The magnitude and frequency dependences of the circular edge current shown in the inset to Fig. 13 are in agreement with the developed theory. The only adjustable parameter is a scattering time in the vicinity of the edge. The scattering times determined by this method for each sample edge are quite close to the average bulk scattering time, the deviations most likely reflect fluctuations of the local scattering time and hence inhomogeneities in the distribution of scatterers. Actually, measurements of chiral edge currents provide very sensitive method of mapping the scattering processes at the edges. Moreover, the sign of the current reflects the type of the charge carriers in the close vicinity of the edge. This feature allowed us to conclude, that the edges of the $n$-type epitaxial graphene are, in fact, $p$-type. The latter, at first glance, surprising result agrees with analysis of the spatially resolved Raman measurements indicating an enhanced density of $p$-type carriers at graphene edges [133,134], transport measurements reporting on the transition from $n$-to $p$-type of doping at the edges of graphene flakes on $\mathrm{SiO}_{2}$ [135] and growth details of epitaxial graphene $[61,116,117,136]$. The data reveals that the measurements of edge currents may serve as a local probe of edge properties of graphene even at the room temperature.

As noted above chiral edge photogalvanic current has also been observed in small-area exfoliated graphene layers [59]. In this case the spot size of the terahertz laser radiation of $1 \mathrm{~mm}^{2}$ is much larger than the micron sized exfoliated flakes and the current is caused by both edge photogalvanic and photon drag (dynamic Hall) effect. Examples of the current helicity 

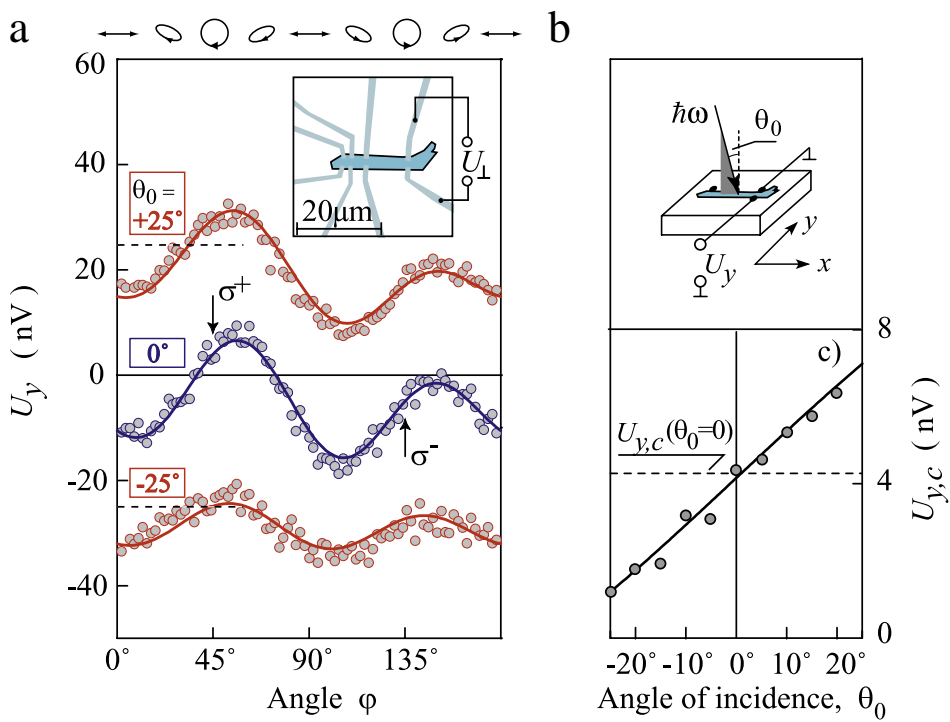

Fig. 14. (a) Photosignal, $U_{y} \propto j_{y}$, in a single graphene sheet as a function of the angle $\varphi$, measured for various angles of incidence, $\theta_{0}$. The data are obtained applying radiation of the $c w$ THz laser with the photon energy $10.5 \mathrm{meV}$, power $\approx 20 \mathrm{~mW}$ and a diameter of the laser spot about $1 \mathrm{~mm}$. The data for $\theta_{0}= \pm 25^{\circ}$ are shifted by $\pm 25 \mathrm{nV}$ for clarity. The horizontal dashed lines show $x$-axes for the shifted data $\left(U_{y}=0\right)$. Full lines are fits to Eq. (45). These fits can be obtained by the superposition of the photon drag effect at oblique incidence given by Eq. (11a) and the edge photogalvanic effect at normal incidence, Eq. (14). The inset shows the sample geometry. The ellipses on top of the left panel illustrate the states of polarization for various angles $\varphi$. Panel (b) illustrates the experimental configuration. (c) Signals due to circularly polarized radiation $U_{y, C}=\left[U\left(\sigma^{+}, \varphi=45^{\circ}\right)-U\left(\sigma^{-}, \varphi=135^{\circ}\right)\right] / 2$ measured as a function of the incidence angle $\theta_{0}$. After [59].

dependence are shown in Fig. 14(a). At normal incidence the data can be well fitted by

$$
J=A_{n} \sin 2 \varphi+B_{n} \sin 4 \varphi+C_{n} \cos 4 \varphi+D_{n}
$$

where $A_{n}, B_{n}, C_{n}$ and $D_{n}$ are coefficients. For oblique incidence the functional behavior remains unchanged but the individual coefficients at the second and the fourth harmonics of the angle $\varphi$ change. The overall behavior at any angle of incidence is well described by the superposition of the edge photogalvanic and dynamic Hall effects of a comparable strength given by Eqs. (11), (14). The contributions can easily be distinguished by measuring the signal as a function of the angle of incidence. This is illustrated in Fig. 14(b) for the circular photocurrent where its dependence on the angle of incidence is plotted. While the photosignal generated at normal incidence is solely determined by the edge photogalvanic current $j \propto \cos \theta_{0}$, the dynamic Hall effect is given by $j \propto \sin \theta_{0} \cos \theta_{0}$, the latter is odd in the angle of incidence and shows up at larger values of $\theta_{0}$.

\subsection{Summarizing remarks on the second order effects in graphene}

The analysis of second order nonlinear effects shows that the most prominent of them such as second harmonics generation, photon drag and photogalvanic effects have been observed in graphene and there functional behavior is in a good agreement with the state-of-the-art theory. Phenomenological and microscopic theories addressed above help to choose the proper experimental geometry and find key parameters of graphene layer and radiation, allowing to excite and optimize second order nonlinearities.

As to the quantitative comparison of the experiments and theory as well as of the results obtained on graphene samples and other well known three- and two-dimensional materials, it still remains a challenging task. The problem is that a systematic experimental study of these phenomena in graphene is not done as yet. The strength of the second order effects substantially changes upon variation of the sample temperature, mobility, carrier density, as well as radiation frequency and its polarization state. The results published so far, to the best of our knowledge, are mostly related with the experiments limited to room temperature and structures with rather low mobility and high value of the Fermi level. Concerning the SHG, the theoretical estimations (see Section 5.1.1 and references therein for details) reveal, that the effect in graphene should be strongly enhanced compared to well known nonlinear crystals. The fact of the experimental observation of SHG signal from the atomically thin carbon layers (Section 5.1.2) indirectly confirms this expectation. The quantitative analysis has been carried out for the photoelectric effects where magnitudes of the photocurrent were possible to measure. As shown in Sections 5.2-5.4 linear and circular photon drag effects, as well as photogalvanic effects observed for pristine graphene and graphene edges are in a very good agreement with the developed theory. The comparison of the photoelectric effects with their counterparts in bulk semiconductors and quantum well structures is, generally, not an easy task because experiments whose conditions differ by material only are not performed so far. A clear enhancement is only observed for the circular photon drag effect. The strength of this photocurrent, steaming from the simultaneous transfer of the photon linear and angular momenta, is about two orders of magnitude larger than that previously detected for GaAs quantum wells [90]. In fact both experiment and theory yield the value of the circular photon drag effect in graphene at room temperature of about $10 \mathrm{nA} /(\mathrm{W} \mathrm{cm})$ while in GaAs quantum well structure the current of $0.01 \mathrm{nA} /(\mathrm{W} \mathrm{cm})$ was reported. By contrast, the linear 
photon drag effect measured in graphene is comparable or somewhat smaller to that detected in bulk Ge and Si crystals. We emphasize, however, that the photon drag voltage in the latter case has picked-up in crystals of several centimeter length whereas similar response in graphene is obtained from a monoatomic layer only. While photon drag effect is comparable or even enhanced compared to other systems, the photogalvanic response in graphene is generally smaller compared to noncentrosymmetric crystals or quantum wells for two reasons. First of all, PGE requires symmetry reduction and, hence, it needs a strong inequivalence of $z$ and $-z$ directions. The magnitude of the effect is proportional to degree of structure asymmetry, which imposes a restriction on the graphene structures which should be deposited on the substrate or have a strong structure inversion asymmetry due to, e.g., adatoms. Second, the PGE in pristine graphene requires action of the $z$-component of the electric field on confined electrons, the effect strongly suppressed for two-dimensional carriers, which are almost unaffected by $E_{z}$, see Section 5.3.1. Consequently the magnitude of the linear and circular photogalvanic effects, being of the order of $20 \mathrm{pA} /(\mathrm{W} \cdot \mathrm{cm})$ at $\lambda \approx 10 \mu \mathrm{m}$, is several tens times smaller than that detected in bulk crystals and QWs $[33,82-84]$. The only exception is the edge photogalvanic effects. Due to local symmetry reduction at the graphene edges the effect is excited by an in-plain electric fields and its magnitude becomes of the order of $0.1 \mathrm{nA} / \mathrm{W}$, which is comparable to the reported values of conventional photogalvanic effects for most of quantum well structures [33,82-84].

\section{Third order effects}

\subsection{Phenomenological discussion}

We continue the discussion of nonlinear high-frequency radiation phenomena excited in graphene by turning to the effects, where induced electric current is proportional to the third power of electromagnetic field. These phenomena are, in general, related to the class of the four-wave mixing effects, where three waves of different frequencies, $\omega_{1}, \omega_{2}$, and $\omega_{3}$, interact and give rise to the fourth one [27]. Such a situation is described by the general relation

$$
j_{\alpha}(\boldsymbol{r}, t)=\sigma_{\alpha \beta \gamma \delta}^{(3, \mathrm{~g})}\left(\omega_{1}, \omega_{2}, \omega_{3}\right) E_{\beta}\left(\omega_{1}, \boldsymbol{q}_{1}\right) E_{\gamma}\left(\omega_{2}, \boldsymbol{q}_{2}\right) E_{\delta}\left(\omega_{3}, \boldsymbol{q}_{3}\right) \mathrm{e}^{-\mathrm{i}\left(\omega_{1}+\omega_{2}+\omega_{3}\right) t+\mathrm{i}\left(\boldsymbol{q}_{1}+\boldsymbol{q}_{2}+\boldsymbol{q}_{3}\right) \boldsymbol{r}}+\text { c.c. },
$$

where $\boldsymbol{q}_{1}, \boldsymbol{q}_{2}$ and $\boldsymbol{q}_{3}$ are corresponding wavevectors of the waves and $\sigma_{\alpha \beta \gamma \delta}^{(3, \mathrm{~g})}\left(\omega_{1}, \omega_{2}, \omega_{3}\right)$ is the general third order conductivity. In the field of nonlinear optics it is usual to write similar to Eq. (46a) expression for the media polarization $\boldsymbol{P}(\boldsymbol{r}, t)$ :

$$
P_{\alpha}(\boldsymbol{r}, t)=\chi_{\alpha \beta \gamma \delta}^{(3, \mathrm{~g})}\left(\omega_{1}, \omega_{2}, \omega_{3}\right) E_{\beta}\left(\omega_{1}, \boldsymbol{q}_{1}\right) E_{\gamma}\left(\omega_{2}, \boldsymbol{q}_{2}\right) E_{\delta}\left(\omega_{3}, \boldsymbol{q}_{3}\right) \mathrm{e}^{-\mathrm{i}\left(\omega_{1}+\omega_{2}+\omega_{3}\right) t+\mathrm{i}\left(\boldsymbol{q}_{1}+\boldsymbol{q}_{2}+\boldsymbol{q}_{3}\right) \boldsymbol{r}}+\text { c.c. },
$$

where the third order susceptibility $\chi_{\alpha \beta \gamma \delta}^{(3, \mathrm{~g})}\left(\omega_{1}, \omega_{2}, \omega_{3}\right)$ is introduced. Taking into account standard relation Eq. (17) between the current density and the polarization one obtains ${ }^{9}$

$$
\sigma_{\alpha \beta \gamma \delta}^{(3, \mathrm{~g})}\left(\omega_{1}, \omega_{2}, \omega_{3}\right)=-\mathrm{i}\left(\omega_{1}+\omega_{2}+\omega_{3}\right) \chi_{\alpha \beta \gamma \delta}^{(3, \mathrm{~g})}\left(\omega_{1}, \omega_{2}, \omega_{3}\right) .
$$

It is assumed in Eqs. (46) that frequencies may take both positive and negative values, the corresponding fields being related through $\boldsymbol{E}^{*}(\omega, \boldsymbol{q})=\boldsymbol{E}(-\omega,-\boldsymbol{q})$, the wavevector dependence of $\sigma^{(3, \mathrm{~g})}$ and $\chi^{(3, \mathrm{~g})}$ is omitted to shorthand the notations. It is worth to mention, that under spatial inversion both current components, $j_{\alpha}$, and cubic combinations, $E_{\beta} E_{\gamma} E_{\delta}$, change their sign, therefore third order effects take place in even centrosymmetric systems without allowance for the radiation wavevector, $\boldsymbol{q}$. Moreover, as we addressed in Section 2 in graphene the third order response is possible for the normal incidence of radiation, where the field has only in-plane components: $E_{x} \neq 0, E_{y} \neq 0, E_{z}=0$, and the current and/or polarization is induced in the plane of the structure.

\subsubsection{Effects of static and ac fields}

It is convenient to start the analysis of the third-order effects from the case, where one of the fields is static, $\boldsymbol{E}(0,0)$. One example of such effects is the electric field induced second harmonic generation, observed recently for monolayer graphene samples [51]. Symmetry analysis of this effect is the same as that of the photon wavevector induced SHG [106], see Section 3.4, with the replacement of the wavevector components $q_{\alpha}$ by the components of the static field $E_{\alpha}(0,0)$. In particular, in the strictly two-dimensional model, the phenomenological relations describing electric field induced second harmonic generation are given by Eq. (16) where the components of the photon wavevector $q_{x}, q_{y}$ should be replaced by the components of the static field $E_{x}(0,0), E_{y}(0,0)$.

Another particular example is the photoconductivity phenomenon, resulting in the $d c$ current proportional to the intensity of the radiation at frequency $\omega$ and the static field $\boldsymbol{E}(0,0)[32]$ :

$$
j_{\alpha}(\boldsymbol{r}, t)=\sigma_{\alpha \beta \gamma \delta}^{\left(3^{\prime \prime}\right)} E_{\beta}(\omega, \boldsymbol{q}) E_{\gamma}^{*}(\omega, \boldsymbol{q}) E_{\delta}(0,0)
$$

\footnotetext{
9 This relation becomes ambiguous if the response is static, $\omega_{1}+\omega_{2}+\omega_{3}=0$. In this case current generation and dielectric polarization becomes independent, cf. Section 3.5 where optical rectification was discussed.
} 

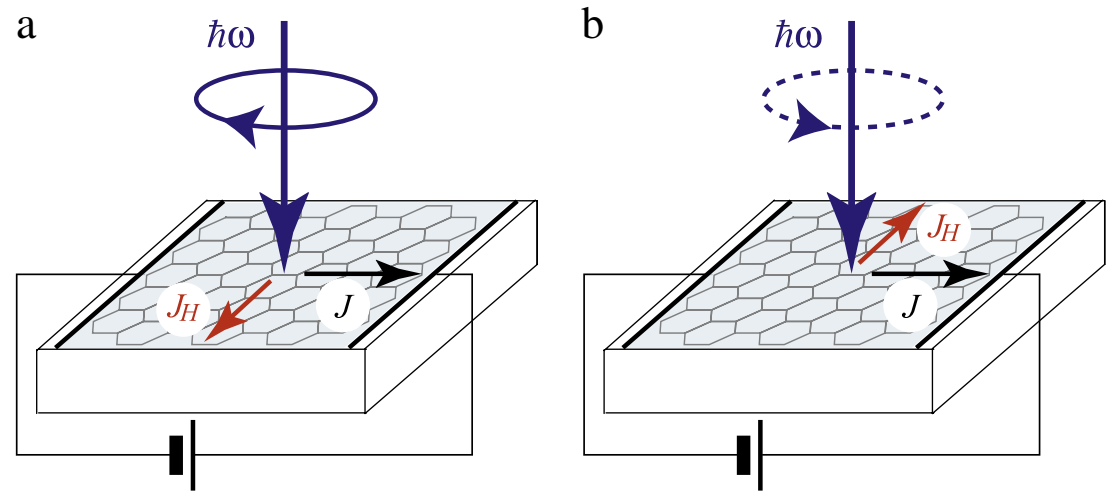

Fig. 15. Schematic illustration of the Hall effect excited by right (a) and left (b) circularly polarized radiation in a biased graphene sample. In the conducting layers the electric current $\boldsymbol{J}$ together with the electric field rotating at frequency $\omega$ the transverse component of the current, $\boldsymbol{J}_{H}$, appears, whose sign reverses with radiation helicity. After [142].

with $\sigma_{\alpha \beta \gamma \delta}^{\left(3^{\prime \prime}\right)} \equiv \sigma_{\alpha \beta \gamma \delta}^{(3, g)}(\omega,-\omega, 0)$. The photoconductivity effects were studied in graphene theoretically and experimentally in a number of works [124,137-141]. Like photon drag effect, the photoconductivity is described by the fourth rank tensor $\sigma_{\alpha \beta \gamma \delta}^{\left(3^{\prime \prime}\right)}$. It can be separated into the symmetric and antisymmetric with respect to $\beta \gamma \leftrightarrow \gamma \beta$ permutation parts giving rise to linear and circular photoconductivities, respectively. Anisotropic linear photoconductivity was discussed theoretically in detail in Ref. [139]. Circular photoconductivity effect also called photovoltaic Hall effect was predicted for graphene in Ref. [142], see also [143-148]. It is schematically depicted in Fig. 15. This effect results in the $d c$ current flow perpendicularly to the static electric field under normal incidence of radiation, $\hat{\boldsymbol{e}} \| z$ :

$$
\boldsymbol{j} \propto\left[\boldsymbol{E}(0,0) \times\left[\boldsymbol{E}(\omega, \boldsymbol{q}) \times \boldsymbol{E}^{*}(\omega, \boldsymbol{q})\right]\right] \propto\left[\boldsymbol{E}(0,0) \times P_{\text {circ }} \hat{\boldsymbol{e}}\right] .
$$

Eq. (49) demonstrates that the pseudovector of radiation circular polarization $P_{\text {circ }} \hat{\boldsymbol{e}}$ plays a role of the magnetic field in Hall effect, as illustrated in Fig. 15. The direction of the transverse current, Eq. (49), changes its sign if the helicity of the radiation is reversed.

\subsubsection{Effects of ac fields}

The set of the third order effects under study further extends if all components of the incident field oscillate with time. First, let us consider a situation, where the sample is illuminated with the single monochromatic wave of a frequency $\omega$, Eq. (1). In this case third order response can be written as

$$
j_{\alpha}(\boldsymbol{r}, t)=\sigma_{\alpha \beta \gamma \delta}^{\left(3^{\prime}\right)} E_{\beta}(\omega, \boldsymbol{q}) E_{\gamma}(\omega, \boldsymbol{q}) E_{\delta}(\omega, \boldsymbol{q}) \mathrm{e}^{-3 \mathrm{i} \omega t+3 \mathrm{i} \boldsymbol{q} r}+\sigma_{\alpha \beta \gamma \delta}^{(3)} E_{\beta}(\omega, \boldsymbol{q}) E_{\gamma}^{*}(\omega, \boldsymbol{q}) E_{\delta}(\omega, \boldsymbol{q}) \mathrm{e}^{-\mathrm{i} \omega t+\mathrm{i} \boldsymbol{q r}}+\text { c.c. }
$$

The first term described by the fourth order tensor $\sigma_{\alpha \beta \gamma \delta}^{\left(3^{\prime}\right)} \equiv \sigma_{\alpha \beta \gamma \delta}^{(3, \mathrm{~g})}(\omega, \omega, \omega)$ corresponds to the third harmonic generation, the effect already studied theoretically and observed experimentally for graphene [48,71,149]. Corresponding fourth rank tensor, $\sigma_{\alpha \beta \gamma \delta}^{\left(3^{\prime}\right)}$, is symmetric with respect to $\gamma \delta \leftrightarrow \delta \gamma$ permutation. Hence, from the symmetry point of view its nonzero components are the same as for the corresponding part of tensor $\Phi_{\alpha \beta \gamma \delta}$ in Eq. (8) describing linear photon drag effect. The second term with the fourth rank tensor $\sigma_{\alpha \beta \gamma \delta}^{(3)} \equiv \sigma_{\alpha \beta \gamma \delta}^{(3, \mathrm{~g})}(\omega,-\omega, \omega)$ describes the current at the frequency $\omega$. This effect can be seen as the correction to the ordinary high-frequency conductivity $\sigma_{\alpha \beta}^{(1)}$ in Eq. (2), which is proportional to the radiation intensity. Actually, it represents the two-photon absorption [35].

Now we turn to the situation where the frequencies of incident waves are different. An important example of such phenomena is the coherent injection of ballistic currents also known as coherent photogalvanic effect [150,151]. In this case, the $d c$ current emerges under the illumination of sample with bi-harmonic field with frequencies $\omega$ and $2 \omega$. As we show below in Section 6.2, the current is caused by the quantum mechanical interference of one and two photon absorption processes, in response to $\boldsymbol{E}(2 \omega, 2 \boldsymbol{q})$ and $\boldsymbol{E}(\omega, \boldsymbol{q})$, respectively, and has the following phenomenological form

$$
j_{\alpha}=\bar{\sigma}_{\alpha \beta \gamma \delta}^{(3)} E_{\beta}(2 \omega, 2 \boldsymbol{q}) E_{\gamma}^{*}(\omega, \boldsymbol{q}) E_{\delta}^{*}(\omega, \boldsymbol{q})+\text { c.c. },
$$

where the field acting on the sample is given by:

$$
\boldsymbol{E}(\boldsymbol{r}, t)=\boldsymbol{E}(\omega, \boldsymbol{q}) \mathrm{e}^{-\mathrm{i} \omega t+\mathrm{i} \boldsymbol{q r}}+\boldsymbol{E}(2 \omega, 2 \boldsymbol{q}) \mathrm{e}^{-2 \mathrm{i} \omega t+2 \mathrm{i} \boldsymbol{q} r}+\text { c.c. }
$$

The corresponding nonlinear conductivity tensor, $\bar{\sigma}_{\alpha \beta \gamma \delta}^{(3)} \equiv \sigma_{\alpha \beta \gamma \delta}^{(3, \mathrm{~g})}(2 \omega,-\omega,-\omega)$, is symmetric with respect to the permutation of two last subscripts $\gamma \delta \leftrightarrow \delta \gamma$, hence, the phenomenological description of this effect in graphene is similar to that of linear photon drag effect and of the second harmonic generation. In particular, in strictly two-dimensional model, the 
coherent photogalvanic effect is described by two independent constants, $M_{1}$ and $M_{2}$ [cf. Eqs. (16)]:

$$
\begin{aligned}
j_{x}= & M_{1} E_{x}(2 \omega, 2 \boldsymbol{q})\left[\left(E_{x}^{*}(\omega, \boldsymbol{q})\right)^{2}+\left(E_{y}^{*}(\omega, \boldsymbol{q})\right)^{2}\right] \\
& +M_{2} E_{x}(2 \omega, 2 \boldsymbol{q})\left[\left(E_{x}^{*}(\omega, \boldsymbol{q})\right)^{2}-\left(E_{y}^{*}(\omega, \boldsymbol{q})\right)^{2}\right]+2 M_{2} E_{y}(2 \omega, 2 \boldsymbol{q}) E_{x}^{*}(\omega, \boldsymbol{q}) E_{y}^{*}(\omega, \boldsymbol{q})+\text { c.c., } \\
j_{y}= & M_{1} E_{y}(2 \omega, 2 \boldsymbol{q})\left[\left(E_{x}^{*}(\omega, \boldsymbol{q})\right)^{2}+\left(E_{y}^{*}(\omega, \boldsymbol{q})\right)^{2}\right] \\
& +M_{2} E_{y}(2 \omega, 2 \boldsymbol{q})\left[\left(E_{y}^{*}(\omega, \boldsymbol{q})\right)^{2}-\left(E_{x}^{*}(\omega, \boldsymbol{q})\right)^{2}\right]+2 M_{2} E_{x}(2 \omega, 2 \boldsymbol{q}) E_{x}^{*}(\omega, \boldsymbol{q}) E_{y}^{*}(\omega, \boldsymbol{q})+\text { c.c. }
\end{aligned}
$$

If the static field $\boldsymbol{E}(0,0)$ in Eq. (49) is replaced by the linearly polarized ac field $\boldsymbol{E}\left(\omega^{\prime}, \boldsymbol{q}^{\prime}\right)$, the transverse (Hall) current appears to be oscillating at the frequency $\omega^{\prime}$. In such a case, the polarization plane of the $a c$ field reflected from or transmitted through the sample rotates, the direction of rotation is determined by the circular polarization of the field $\boldsymbol{E}(\omega, \boldsymbol{q})$. This effect can be termed by optically induced Faraday/Kerr effect similarly to the Faraday/Kerr rotation by optically induced spin polarization in semiconductors [152].

Below we briefly discuss theoretical approaches to calculate the third order effects and available experimental data.

\subsection{Theoretical background}

The microscopic mechanisms of the third order response are dominated by the energy spectrum nonparabolicity effect [34,47,153]: As already noted in Section 2, the electron velocity in graphene $\boldsymbol{v}$ depends nonlinearly on the electron momentum $\boldsymbol{p}$, see Eq. (20), hence, harmonic oscillations of $\boldsymbol{p}$ driven by external electromagnetic field result in the anharmonic response in the velocity and in the electric current, that is, in frequency conversion [153].

Since for the third-order effects neither the allowance for the radiation wavevector nor the account of its magnetic field is needed, its description is quite straightforward in the classical frequency range, $\hbar \omega \ll E_{F}$. We employ the kinetic equation for momentum and time dependent distribution function:

$$
\frac{\partial f}{\partial t}+e \boldsymbol{E}(t) \frac{\partial f}{\partial \boldsymbol{p}}=-\frac{f(\boldsymbol{p}, t)-f_{0}(\boldsymbol{p})}{\tau},
$$

where the simplest form of the collision integral is taken, $f_{0}(\boldsymbol{p})$ is the equilibrium distribution function. Its solution, which takes into account electric field to all orders can be written as [154]

$$
f(\boldsymbol{p}, t)=f_{0}\left[\boldsymbol{p}-\boldsymbol{p}_{0}(t)\right] \mathrm{e}^{-t / \tau}+\frac{1}{\tau} \int_{-\infty}^{t} \mathrm{~d} t^{\prime} \mathrm{e}^{-\frac{t-t^{\prime}}{\tau}} f_{0}\left[\boldsymbol{p}-\boldsymbol{p}_{0}(t)+\boldsymbol{p}_{0}\left(t^{\prime}\right)\right],
$$

where $\boldsymbol{p}_{0}(t)=\int_{-\infty}^{t} e \boldsymbol{E}(t) \mathrm{d} t$ is the electron momentum acquired from the field and it is assumed that the field was turned on at $t \rightarrow-\infty$. Eq. (55) extends the treatment developed in Refs. [47,153] for ballistic electrons to allow for the scattering. Correspondingly, the induced electric current at zero temperature for degenerate electrons in graphene with density $n$ can be written at $t \gg \tau$ as

$$
\boldsymbol{j}=e n v \int_{-\infty}^{t} \frac{\mathrm{d} t^{\prime}}{\tau} \mathrm{e}^{-\frac{t-t^{\prime}}{\tau}} \frac{\boldsymbol{P}}{\sqrt{1+P^{2}}} g\left(\frac{2 P}{1+P^{2}}\right),
$$

where $\boldsymbol{P} \equiv \boldsymbol{P}\left(t, t^{\prime}\right)=\left[\boldsymbol{p}_{0}(t)-\boldsymbol{p}_{0}\left(t^{\prime}\right)\right] / p_{F}, p_{F}$ is the Fermi wavevector and function $g(x)$ is related with hypergeometric function as

$$
g(x)={ }_{2} \mathrm{~F}_{1}\left(\frac{1}{4}, \frac{3}{4}, 2, x^{2}\right) .
$$

Decomposing Eq. (56) up to the third order of $\boldsymbol{P}$ we obtain the following expression for the nonlinear response:

$$
\boldsymbol{j}=e N_{0} v \int_{-\infty}^{t} \frac{\mathrm{d} t^{\prime}}{\tau} \mathrm{e}^{-\frac{t-t^{\prime}}{\tau}}\left(\boldsymbol{P}-\frac{1}{8} \boldsymbol{P} P^{2}\right) .
$$

Here $N_{0}$ is the electron density, the first term in parentheses describes linear response and second one describes the third order effects.

For example, for the incident harmonic radiation $\boldsymbol{E}=\boldsymbol{E}_{0} \mathrm{e}^{-\mathrm{i} \omega t}$ the term $\boldsymbol{P} P^{2}$ is oscillating at $3 \omega$ with the result

$$
\boldsymbol{j}(3 \omega)=-e^{4} N_{0} v^{4} \frac{3 \boldsymbol{E}_{0} E_{0}^{2}}{4 E_{F}^{3}} \tau_{\omega} \tau_{2 \omega} \tau_{3 \omega},
$$

with $\tau_{\omega}=\tau /(1-i \omega \tau)$. In the limit $\omega \tau \gg 1$ (but $\left.\hbar \omega \ll E_{F}\right)$ Eq. (58) agrees with Eq. (9) of [47].

As an example of the static field induced second harmonic generation we consider simplest situation where the static field $\boldsymbol{E}(0,0)=\boldsymbol{E}_{0} \| x$, while the alternating (radiation) field $\boldsymbol{E}_{1} \exp (-\mathrm{i} \omega t)+$ c.c. is linearly polarized along $y$ axis, i.e. Stokes 

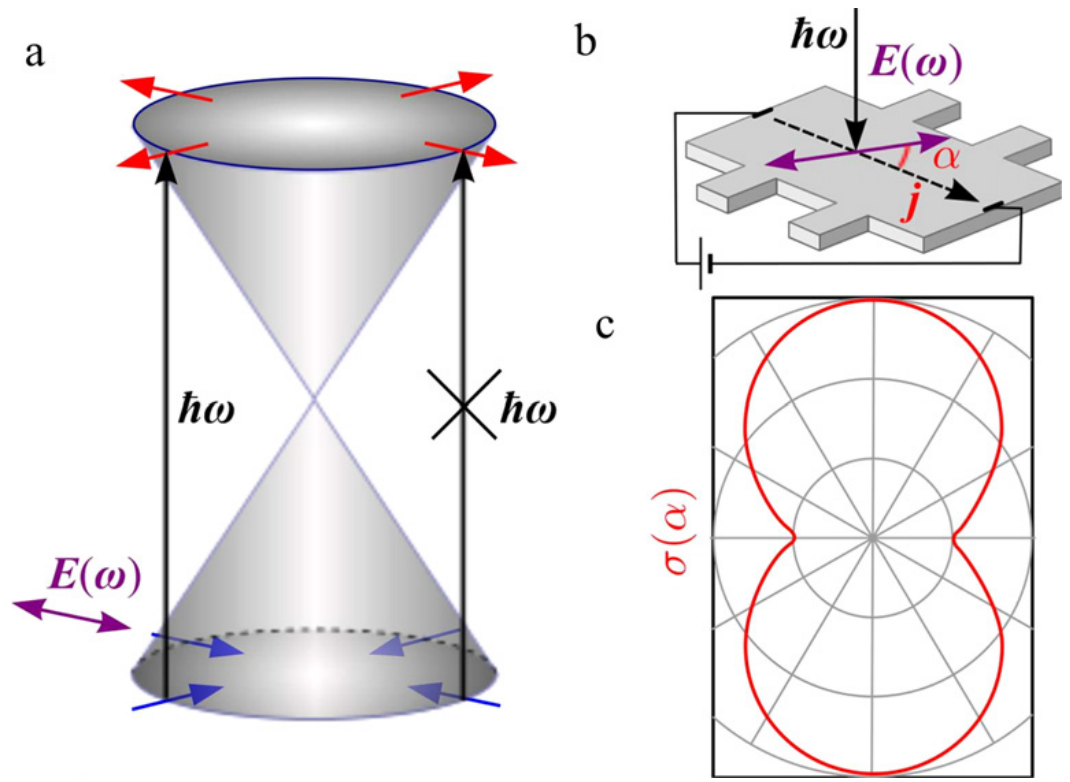

Fig. 16. (a) Scheme of interband optical transitions excited by linearly polarized irradiation with $\hbar \omega \geq 2 E_{F}$. Due to selection rules the transitions are forbidden for $\boldsymbol{k}$ parallel to the linear polarization plane of light. (b) Graphene Hall bar sample irradiated with linearly polarized electromagnetic wave. Application of a bias voltage leads to an electrical current $\boldsymbol{j}$, whose magnitude depends on the in-plane orientation of polarization plane of the linear polarized light given by the azimuthal angle $\alpha$. (c) Photoconductivity, $\sigma(\alpha)$, as a function of $\alpha$, an angle between the current and linear polarization plane of radiation. After [139].

parameters of incident field are $S_{1}=-1, S_{2}=S_{3}=0$. Calculation shows that the current at a double frequency flows along $x$ axis and is given by

$$
\boldsymbol{j}(2 \omega)=e^{4} N_{0} v^{4} \frac{\boldsymbol{E}_{0} E_{1}^{2}}{4 E_{F}^{3}} \tau_{\omega}^{2} \tau_{2 \omega}^{2} \frac{2 \omega^{2} \tau^{2}+6 \mathrm{i} \omega \tau-3}{\tau} .
$$

The microscopic theory of the field induced second harmonic generation for bilayer graphene was developed in Ref. [155] for the quantum frequency range. It was predicted that AB-stacked bilayer graphene can exhibit a giant and tunable second order nonlinear susceptibility if the in-plane electric field is applied. The susceptibility varies from 0 to $10^{5} \mathrm{pm} / \mathrm{V}$ depending on the magnitude of the static field and exceeds by 3 orders of magnitude that of conventional nonlinear crystal AgGaSe ${ }_{2}$. Such a high values of the electric field induced response is related to the specifics of the bilayer band structure, and its detailed consideration is out of the scope of this review.

A detailed theory of linear photoconductivity in graphene for the case of interband optical transitions where the quantum regime of light-matter interaction is realized was developed in Ref. [139]. Following this work we note, that due to the optical selection rules the excitation with linearly polarized light generates the distribution of photocarriers containing second angular harmonic (momentum alignment) whose orientation is determined by the polarization plane of the radiation, see Fig. 16 and Refs. [105,139] for details. Indeed, the interband transitions are forbidden for electron momentum $\boldsymbol{p}$ being parallel to the linear polarization plane of radiation, since the perturbation due to electromagnetic radiation $\propto v(\boldsymbol{\sigma} \cdot \boldsymbol{A})$, where $\boldsymbol{A}$ is the vector potential of radiation does not mix eigenstates of the Dirac Hamiltonian, Eq. (3), with $\boldsymbol{p} \| \boldsymbol{A}$. The matrix element of the interband transition has a form

$$
M \propto p_{x} A_{y}-p_{y} A_{x} .
$$

The distribution function of photoelectrons $\delta f$ is determined by the transition rate $\propto|M|^{2}$, namely,

$$
\delta f \propto p_{x}^{2}\left|E_{x}\right|^{2}+p_{y}^{2}\left|E_{y}\right|^{2}-p_{x} p_{y}\left(E_{x} E_{y}^{*}+E_{x}^{*} E_{y}\right)
$$

where $E_{x}$ and $E_{y}$ are the incident field components. Apart from the isotropic part $\propto\left(\left|E_{x}\right|^{2}+\left|E_{y}\right|^{2}\right)$ the photoelectrons distribution contains second angular harmonics of electron momentum $\boldsymbol{p}: \cos 2 \varphi_{\boldsymbol{p}} \propto\left(\left|E_{x}\right|^{2}-\left|E_{y}\right|^{2}\right)$ and $\sin 2 \varphi_{\boldsymbol{p}} \propto$ $\left(E_{x} E_{y}^{*}+E_{x}^{*} E_{y}\right)$, where $\varphi_{\boldsymbol{p}}$ is the angle between $\boldsymbol{p}$ and $x$ axis. As a result, the magnitude of the current of photoelectrons driven by external bias depends strongly on the mutual orientation of the polarization plane of radiation and external electric field. For the classical frequency range the second-order in the ac field correction to the distribution function assumes the same form of Eq. (61) giving rise to the anisotropic photoconductivity.

The description of the coherent photogalvanic and frequency mixing phenomena can be carried out along the same lines for the classical range of frequencies. As a particular example we consider bichromatic field in the form

$$
\boldsymbol{E}=\boldsymbol{E}_{1} \cos \omega t+\boldsymbol{E}_{2} \cos (2 \omega t+\delta),
$$

incident on the sample. The parameter $\delta$ describes the phase shift between $\omega$ and $2 \omega$ fields. In the geometry $\boldsymbol{E}_{1}\left\|\boldsymbol{E}_{2}\right\| x$ the $x$-component of the $d c$ current described by phenomenological parameter $M_{1}$ in Eqs. (53) yields 


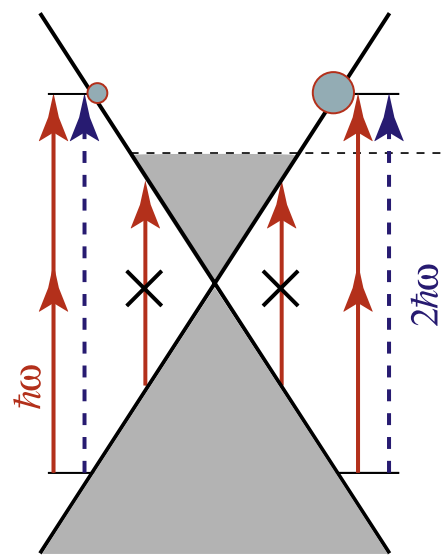

$\mathrm{b}$

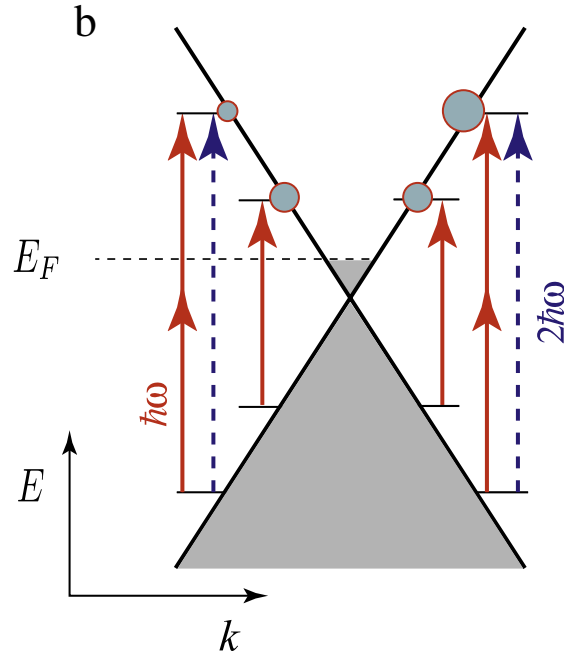

Fig. 17. Optical interband optical transition for (a) $E_{F}<\hbar \omega<2 E_{F}$ and (b) $\hbar \omega>2 E_{F}$. The dotted horizontal lines indicate the Fermi level. Solid arrows denote transitions caused by the beam of frequency $\omega$ and dashed arrows show transitions caused by the $2 \omega$ beam. Filled circles of various diameters sketch asymmetric electron populations at $\pm k$ caused by the quantum interference of a single and two-photon absorption processes. The photoinduced imbalance in $\boldsymbol{k}$-space causes dc current generation. After [63].

$$
j_{x}=-e^{4} N_{0} v^{4} \frac{9 E_{1}^{2} E_{2} \cos \delta}{16 E_{F}^{3}} \frac{\tau^{3}}{1+5 \omega^{2} \tau^{2}+4 \omega^{4} \tau^{4}} .
$$

If, by contrast, $\boldsymbol{E}_{1}\left\|y \perp \boldsymbol{E}_{2}\right\| x$, then the $d c$ photocurrent described by phenomenological parameter $M_{2}$ in Eqs. (53) has form

$$
j_{x}=-e^{4} N_{0} v^{4} \frac{3 E_{1}^{2} E_{2} \cos \delta}{16 E_{F}^{3}} \frac{\tau^{3}}{1+5 \omega^{2} \tau^{2}+4 \omega^{4} \tau^{4}} .
$$

Note that, similar to Eq. (63a) expression was derived in Ref. [156] (see also [154]) for the semiconductor system with nonparabolic energy dispersion. It follows from Eq. (63a) that the coherent photocurrent is extremely sensitive to the phase relation between two waves: The current is proportional to the cosine of the phaseshift. It is worth to mention that in the ballistic case $(\tau \rightarrow \infty)$ the first term in Eq. (55) also gives rise to the current $\propto \omega^{-3} \sin \delta$, see Ref. [156] for the semiconductor system with nonparabolic dispersion and Ref. [157] for "mini-gapped" graphene on a substrate. The mechanism of the coherent photogalvanic effect in the systems with parabolic dispersion is presented in Ref. [151].

In the quantum frequency range, $\omega \tau \gg 1, \hbar \omega \sim E_{F}$ (or even $\hbar \omega \gg E_{F}$ ) the description of the third order phenomena can be carried out in a similar fashion. Instead of applying Boltzmann equation (54) one may use similar equation for the density matrix where the collision integral is absent. Such a treatment is outlined in Refs. $[40,55,63,158-160]$. In the case of $i n t r a b a n d$ transitions, where the double photon energy exceeds $2 E_{F}$ and two-photon transition becomes possible, see Fig. 17, the coherent photogalvanic effect can be understood in terms of quantum interference of single and two photon processes [63]. These processes are schematically shown in Fig. 17. To begin with, consider the case where the direct absorption of a single photon with the frequency $\omega$ is forbidden, as illustrated in Fig. 17(a). The matrix element describing the electron transition from the valence to the conduction band caused by the absorption of one photon with the frequency $2 \omega$ is linear in the electron wavevector $\boldsymbol{k}$ and has a form

$$
M_{2 \omega}^{(1)} \propto k_{x} A_{y}(2 \omega)-k_{y} A_{x}(2 \omega) .
$$

Here $\boldsymbol{A}(2 \omega)=\left[A_{x}(2 \omega), A_{y}(2 \omega)\right]$ is the vector potential of the field oscillating at $2 \omega$. Due to the condition $\hbar \omega<2 E_{F}$ the direct interband absorption of the radiation with the frequency $\omega$ is possible only via the two-photon absorption. Such a secondorder process takes place via the intermediate states in the same band, yielding the matrix element of the two-photon process in the form

$$
M_{\omega}^{(2)} \propto\left[k_{x} A_{y}(\omega)-k_{y} A_{x}(\omega)\right]\left[k_{x} A_{x}(\omega)+k_{y} A_{y}(\omega)\right],
$$

with $\boldsymbol{A}(\omega)=\left[A_{x}(\omega), A_{y}(\omega)\right]$ being the vector potential of $\omega$-oscillating field. As both, one $2 \omega$ photon absorption and two $\omega$ photon absorption, processes mix the same states they interfere. The total absorption rate is proportional to the $\left|M_{2 \omega}^{(1)}+M_{\omega}^{(2)}\right|^{2}$ with the interference contribution in the form

$$
\propto 2 \operatorname{Re}\left[M_{2 \omega}^{(1)} M_{\omega}^{(2)^{*}}\right]
$$

which results in the anisotropic distribution of photoelectrons, shown by filled circles of different sizes in Fig. 17(a) and, correspondingly, in the electric current. The magnitude and direction of electric current are controlled by the orientation 


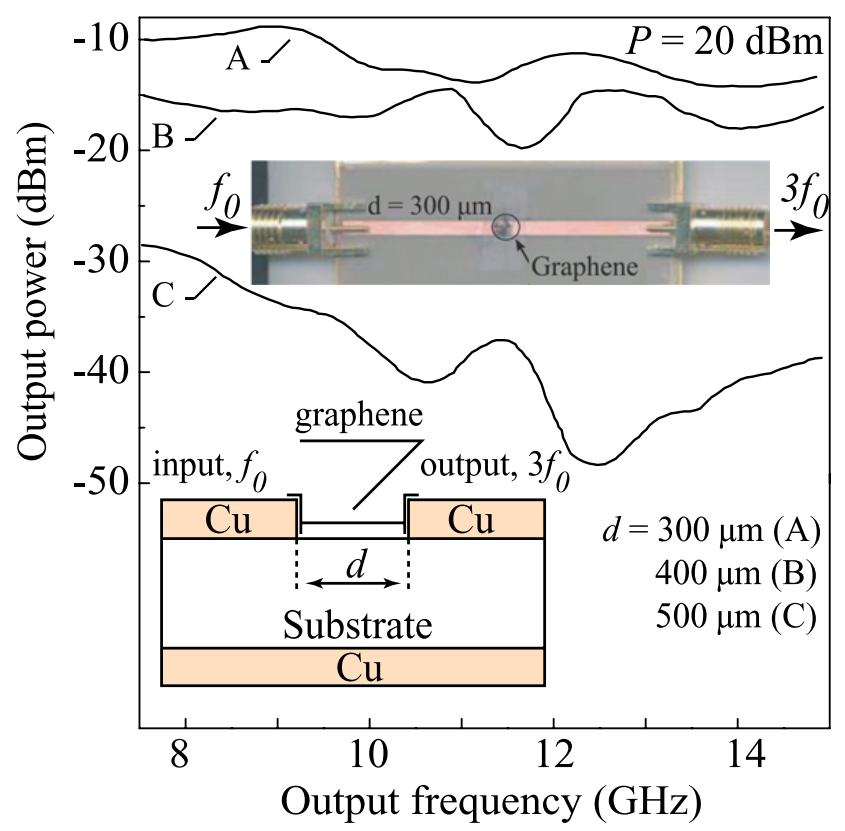

Fig. 18. Radiation output power as a function of output frequency, $3 f_{0}$, for a graphene based frequency tripler. Curves $A, B$ and $C$ present the characteristics of three devices with different gap lengths of $300 \mu \mathrm{m}, 400 \mu \mathrm{m}$, and $500 \mu \mathrm{m}$. Middle inset shows photograph of manufactured device and bottom inset demonstrates the device cross section. After [162].

of $\boldsymbol{A}(\omega), \boldsymbol{A}(2 \omega)$ and their phase difference. Similar situation occurs if $\hbar \omega>2 E_{F}$, i.e. where single photon absorption is also possible, see Fig. 17(b). While the interference of one and two-photon absorption processes is possible and gives rise to the electric current, the absorption of single photon with the frequency $\omega$, although being possible, does not result in the asymmetry of electron distribution and does not lead to current generation. For the case of interband transitions in the quantum frequency range, we can estimate the third order response following Ref. [55] as a product of the linear interband conductivity, $\sim e^{2} / \hbar$, the electric field magnitude $E$ and the square of the small parameter $\left(e E v / \hbar \omega^{2}\right)$, which characterizes the ratio of the work performed by the electric field during one period and the photon energy. The resulting magnitude of the third order current is

$$
j=e \frac{e^{4} v^{2}}{\hbar^{3} \omega^{4}} E^{3}
$$

where the dimensionless parameter $\mathcal{C}$ depends on the frequencies and relative amplitudes of incident waves. An enhancement of the third order response as compared with other nonlinear systems can be clearly seen if one introduces effective nonlinear susceptibility $\chi^{(3)}$, see Ref. [55] for details. The estimations presented there demonstrate that for $\lambda \sim 1 \mu \mathrm{m}$ the value of $\chi^{(3)}$ is about $10^{-7}$ esu (electrostatic units). Moreover, it was demonstrated theoretically in Ref. [161] that the third order non-linearity in graphene can be tuned and enhanced by the magnetic field, where it may reach values up to $10^{-3}$ esu, being strongest among the non-linear materials known so far.

To conclude this section, we present the results of analytical calculations of the optically induced Faraday/Kerr effect introduced in Section 6.1.2. Theoretical estimate of this effect for the classical frequency range can be obtained considering the incident radiation in a form

$$
E_{x}(t)=E_{1} \cos \omega t+E_{2} \cos \omega t, \quad E_{y}(t)=\mp E_{1} \sin \omega t,
$$

corresponding to the combination of the circularly polarized wave with the amplitude $E_{1}$ and linearly polarized wave with the amplitude $E_{2} \ll E_{1}$. Signs $\mp$ in expression for $E_{y}$ correspond to right and left circular polarizations for the wave propagating along negative $z$ axis. It follows from Eq. (57) that the transverse component of the current in the classical frequency range is

$$
j_{y}=\mp e^{4} N_{0} v^{4} \frac{3 E_{1}^{2} E_{2}}{8 E_{F}^{3}} \frac{\tau^{3}}{1+5 \omega^{2} \tau^{2}+4 \omega^{4} \tau^{4}}(2 \omega \tau \cos \omega t-\sin \omega t) .
$$

The appearance of $j_{y} \neq 0$ is responsible for the Faraday rotation of the polarization plane of the transmitted (and Kerr rotation of reflected) probe beam $E_{2}$ incident on the excited by circularly polarized beam $E_{1}$ graphene.

\subsection{Third and higher harmonic generation and frequency mixing: experiment}

The generation of third harmonic and higher orders nonlinearities (up to seventh order harmonic) were reported first for millimeter waves in Ref. [48] where the classical range of frequencies, $\hbar \omega \ll E_{F}$, is realized (this experiment on a monolayer graphene is already described in detail in Section 5.1.2), and in Refs. [52,162]. In the latter work a graphene based frequency tripler was manufactured. The sketch of the setup and photograph of the device is shown in Fig. 18. The nonlinear component 
a

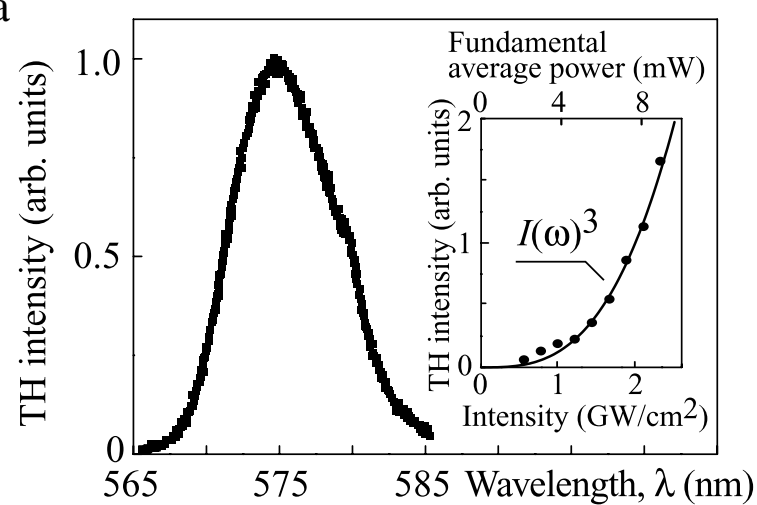

b

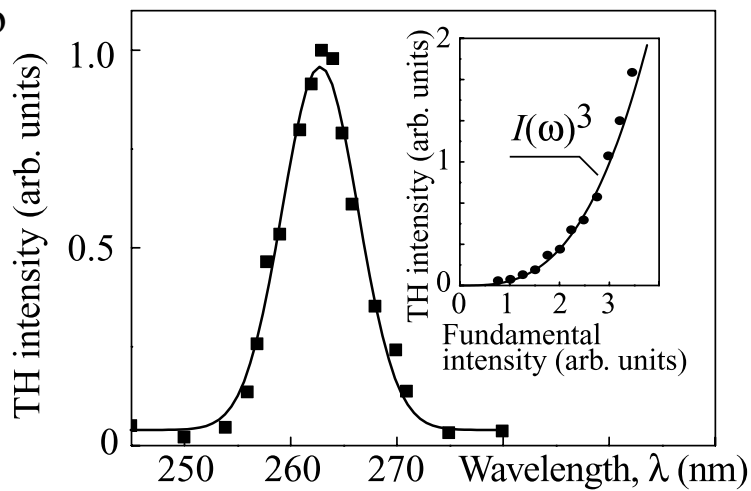

Fig. 19. Spectra of third harmonic generation measured in Ref. [54], panel (a), and Ref. [53], panel (b). Insets show the measured third harmonic (TH) power as a function of the fundamental beam intensity (points). Solid lines show the cubic law fit. After [53,54].

of the device consists of a microstrip line with a small gap covered by a few layer graphene film. A standard microwave setup consisting of a generator tunable in the $2.5-5 \mathrm{GHz}$ range and a spectrum analyzer was used. Output frequencies in the range between 8 and $15 \mathrm{GHz}$ have been obtained with a received output power up to $-10 \mathrm{dBm}$ as shown in the main panel of Fig. 18. Almost flat frequency behavior can be obtained in the whole output frequency range.

Third harmonic was most recently observed in graphene for the fundamental frequency $\omega$ in the near infrared range in two works [54] (exfoliated graphene) and [53] (CVD graphene) for the quantum regime of light-matter coupling. In Ref. [54] the fundamental wavelength is $\approx 1.72 \mu \mathrm{m}$ (third harmonic wavelength is $\approx 0.575 \mu \mathrm{m}$ ), while in Ref. [53] the fundamental wavelength was somewhat shorter $\approx 0.8 \mu \mathrm{m}$ (third harmonic corresponds to $\approx 0.265 \mu \mathrm{m}$ ). In the latter case the third harmonic was close to resonance with the optical transition in the $M$ point of the Brillouin zone making it possible to enhance the signal. Fig. 19 demonstrates the spectra of the third harmonic measured in Ref. [54], panel (a), and in Ref. [53], panel (b). The insets demonstrate that the third harmonic intensity indeed scales as cube of fundamental harmonic intensity. According to Ref. [54] the third order susceptibility of graphene for such near-IR frequencies is on the order of $10^{-8}$ esu and is by several order of magnitude larger than in transparent materials.

Besides the third harmonic generation, several other effects caused by the third order nonlinearity have been reported for near-infrared, optical, and UV frequencies. In particular, the $\chi^{(3)}$ have been studied for graphene in solutions by means of the time-resolved pump-probe techniques [71,72]. In Ref. [71] a purely coherent nonlinear optical response of highquality graphene sheets functionalized by alkylamine has been demonstrated. These graphene sheets has been investigated, using near-infrared, visible, and ultraviolet continuous wave and ultrafast laser beams, and spatial self-phase modulation has been observed in the solution dispersions. The ultrafast third-order nonlinear optical properties of graphene in both suspension and film state were studied using femtosecond time resolved optical Kerr gate technique in Ref. [72]. The thirdorder nonlinear optical susceptibility of about $4 \times 10^{-14}$ esu was observed for solution of $0.010 \mathrm{mg} / \mathrm{ml}$. While huge nonlinear response has been detected, the signal may result from the superposition of the nonlinear response of graphene itself and the effect of reorientation and alignment of graphene sheets in solutions induced by the electromagnetic field which is similar to the case of liquid crystals [71]. Thus, the detailed discussion of these interesting and important for application results are out of scope of the present review aimed to pristine graphene and graphene on substrates.

Another experimental manifestation of the third order nonlinearity is a frequency mixing, recently demonstrated for infrared/red light [55] and radiation and GHz frequency range [52]. Fig. 20(a) shows the setup used in Ref. [55] for the visible/infrared four-wave mixing experiments carried out for the quantum regime, which involves the generation of optical frequency harmonics $2 \omega_{1}-\omega_{2}$ under irradiation by two monochromatic waves with the frequencies $\omega_{1}$ and $\omega_{2}$ as depicted in Fig. 20(b). Two incident pump laser beams with wavelengths $\lambda_{1}$ (tunable from 670 to $980 \mathrm{~nm}$ ) and $\lambda_{2}(1130-1450 \mathrm{~nm}$ ) 

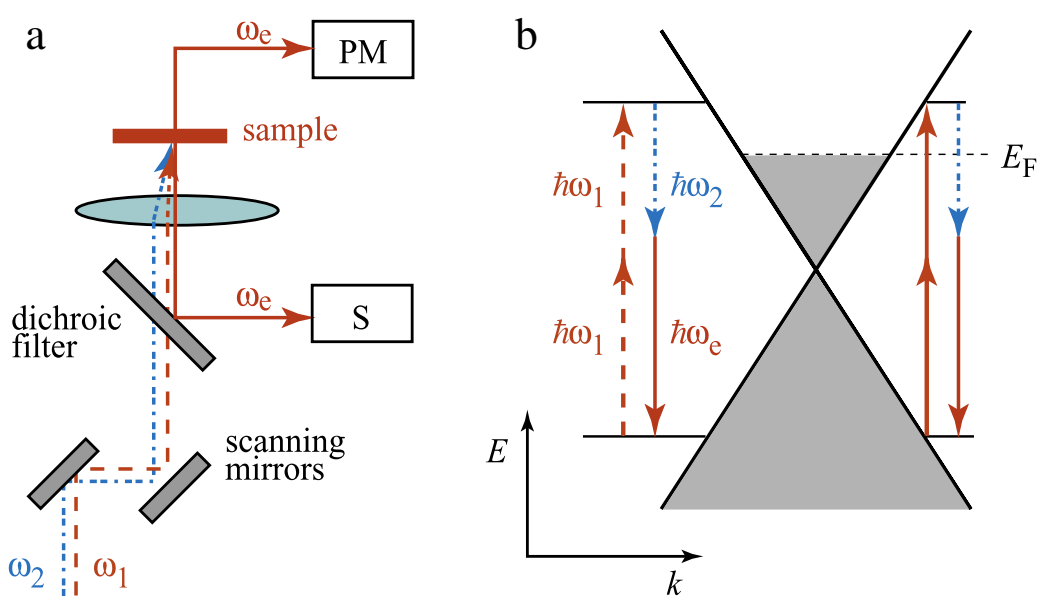

Fig. 20. (a) Schematic illustration of the experimental setup used in the frequency mixing experiments. Emission beam with frequency $\omega_{e}$ caused by mixing of the beams with frequencies $\omega_{1}$ and $\omega_{2}$ is detected by a photomultiplier, PM, and spectrometer, S. (b) Sketch of the frequency mixing effect in graphene with the three resonant photon energies (arrows) involved in the process. After [55].

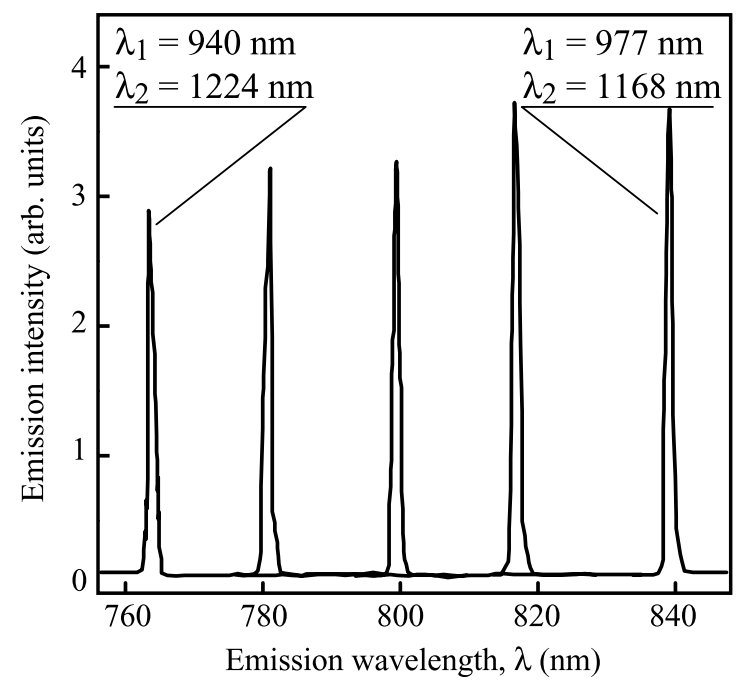

Fig. 21. Emission spectra of an exfoliated graphene flake excited with pump pulses of different wavelengths, $\left(\lambda_{1}, \lambda_{2}\right):(940 \mathrm{~nm}, 1224 \mathrm{~nm}),(950 \mathrm{~nm}$, $1210 \mathrm{~nm}),(958 \mathrm{~nm}, 1196 \mathrm{~nm}),(967 \mathrm{~nm}, 1183 \mathrm{~nm})$, and (977 nm, $1168 \mathrm{~nm}$ ) from left to right, respectively. After [55].

duration about 6 ps are focused collinearly onto a sample and mix together to generate a third, coherent beam of wavelength $\lambda_{e}$. The incident pump pulses are focused onto the sample using a water immersion objective with a numerical aperture of 1.2, giving rise to a spot size $<1 \mu \mathrm{m}$ and time averaged and peak excitation powers at the sample of about $1 \mathrm{~mW}$ and $10 \mathrm{~W}$, respectively. Note that in these experiments the peak beam power is much higher than in other experiments. This fact indicates that the graphene samples are robust and rather higher power can be used without damaging samples. The nonlinear signal is presented in Fig. 21 for different combinations of incident wavelengths $\lambda_{1}$ and $\lambda_{2}$. The results of Ref. [55] evidence that the graphene has an exceptionally high nonlinear response, with the effective nonlinear susceptibility $\chi^{(3)}=10^{-7}$ esu being by about an order of magnitude larger than that obtained by third harmonic generation in Ref. [54] and by several orders of magnitude larger than that for, e.g., gold or glass. Moreover, this nonlinearity is shown to be almost dispersionless in a wide range of emission wavelengths (from 760 to $840 \mathrm{~nm}$ ). Interestingly, a high third order nonlinearity yields an enormously large contrast between the responses of the sample and substrate as well as between the samples with different number of graphene layers, the latter is due to the linear increase of the $\chi^{(3)}$ with the number of layers in the sample, see Fig. 22(c). This results in much better microscopic images of graphene compared to those obtained in a normal optical reflection as demonstrated in Fig. 22(a) and (b). Further application of graphene signal mixing has been addressed in Ref. [52] where signals with $\mathrm{GHz}$ frequencies were mixed in different combinations by three layer graphene device with linear current-voltage characteristic [160].

\subsection{Coherent injection of ballistic photocurrents: experiment}

Ballistic photocurrents related to the third order nonlinearity have been experimentally demonstrated in the infrared spectral range with $\hbar \omega>E_{F}$, i.e. in the quantum regime of light-matter interaction for multilayer epitaxial graphene film 
(a) green light reflection
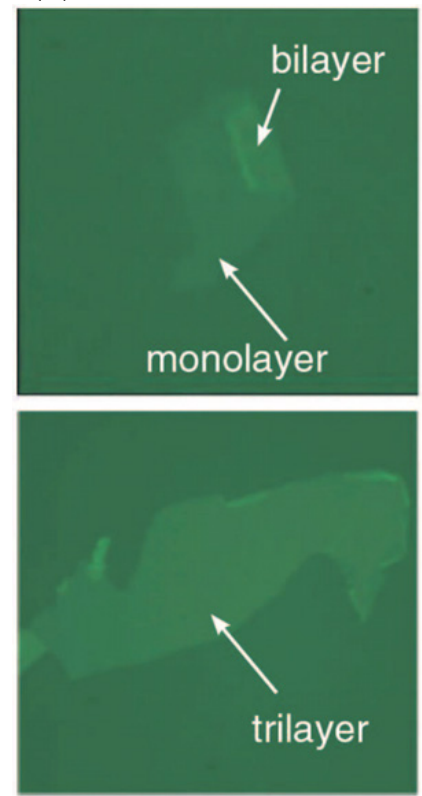

(c)

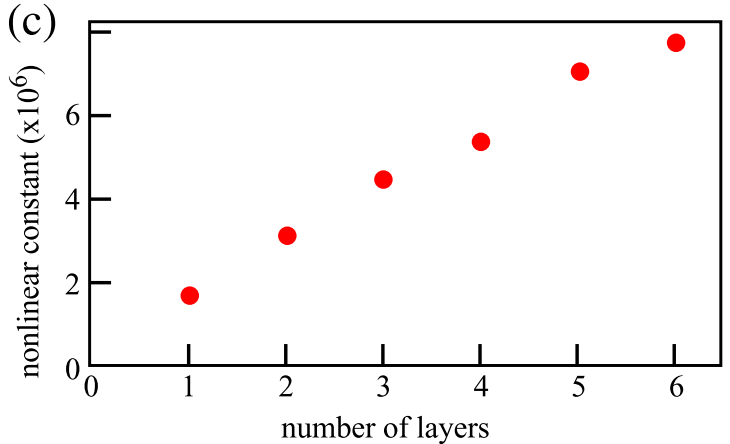

(b) four-wave mixing
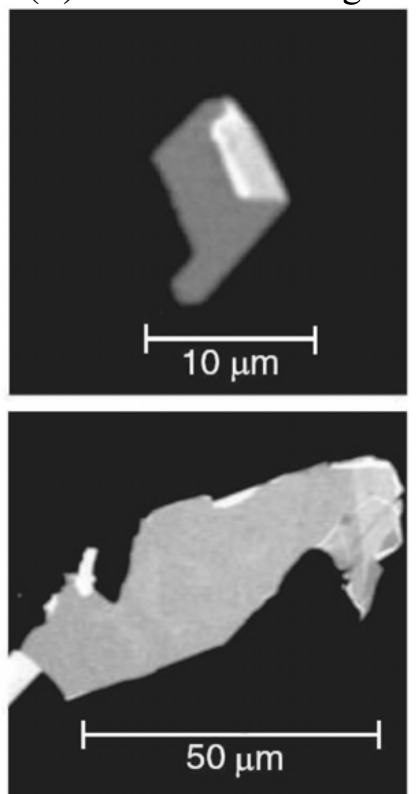

Fig. 22. (a) Green light (550 nm) reflection images of two exfoliated graphene flakes. (b) Nonlinear optical images measured with pump wavelengths of $969 \mathrm{~nm}$ and $1179 \mathrm{~nm}$. Image acquisition times are approximately $0.6 \mathrm{~s}$. (c) The contrast in four-wave mixing images as a function of the number of graphene layers. After [55].

produced on the C-terminated face of single-crystal 4H-SiC [63,64]. In these multilayer epitaxial graphene films the first few layers were heavily doped $\left(10^{13} \mathrm{~cm}^{-2}\right)$ with the doping decreasing rapidly by four orders of magnitude. Interestingly, samples used in these experiments have from 9 to 63 graphene atomic layers; but have been shown by independent studies to have the graphene-like linear band structure and be distinct from bulk graphite [63,163-169]. In order to investigate the coherent photocurrent arising on subpicosecond timescale the $\mathrm{THz}$ radiation emitted by the current pulse has been measured. Application of short current pulses for THz radiation generation (Auston switch) has been developed in early 90's [170,171] and is currently widely used for generation of $\mathrm{THz}$ radiation and time-domain $\mathrm{THz}$ spectroscopy $[33,172,173]$. In this case the generated electric field

$$
\boldsymbol{E}(t) \propto d \boldsymbol{j}(t) / d t \sim \boldsymbol{j} / \tau_{p},
$$

where $\boldsymbol{j}(t)$ is the current pulse density and $\tau_{p}$ is its duration. Since typical current pulse durations correspond to picosecond timescale, the emitted field corresponds to $\mathrm{THz}$ frequency range. Consequently, the dynamics of the emitted THz field reflects behavior of the generated current.

The system used in these experiments consists of pulsed Ti:Sapphire laser, optical parametric amplifier, and a differential frequency generator yielding $200 \mathrm{fs}$ infrared pulses with intensity of $\mathrm{GW} / \mathrm{cm}^{2}$ range. In order to generate the coherent current, which requires two coherent beams at frequencies $\omega$ and $2 \omega$, the beam of the laser operating at a fundamental frequency is split into two beams. One of those is directed to the sample, whereas the second is frequency doubled by the second harmonic generation process in the nonlinear crystal. As a result, the fields at the frequency $\omega$ and $2 \omega$ are coherent and scale with radiation power as $E_{\omega} \propto P_{\omega}^{1 / 2}$ and $E_{2 \omega} \propto P_{2 \omega}^{1 / 2} \propto P_{\omega}$. As described above in Section 6.2, due to the interference of the two-photon transition with the frequency $\omega$ and a single photon transition with the frequency $2 \omega$, the current is generated. This current induces the radiation of THz range, see Eq. (70). An example of the power dependence of the emitted $\mathrm{THz}$ radiation for the fundamental beam wavelength of the $4.8 \mu \mathrm{m}$ is shown in Fig. 23. As it is seen from Eq. (70) terahertz radiation signal detected by the method of electro-optical sampling is associated with the two color current injection process and scales with the pump power as $\left|E_{T H z}\right| \propto|j| \propto P_{\omega} P_{2 \omega}^{1 / 2} \propto P_{\omega}^{2}$. The experiment data in Fig. 23 support the expected power 


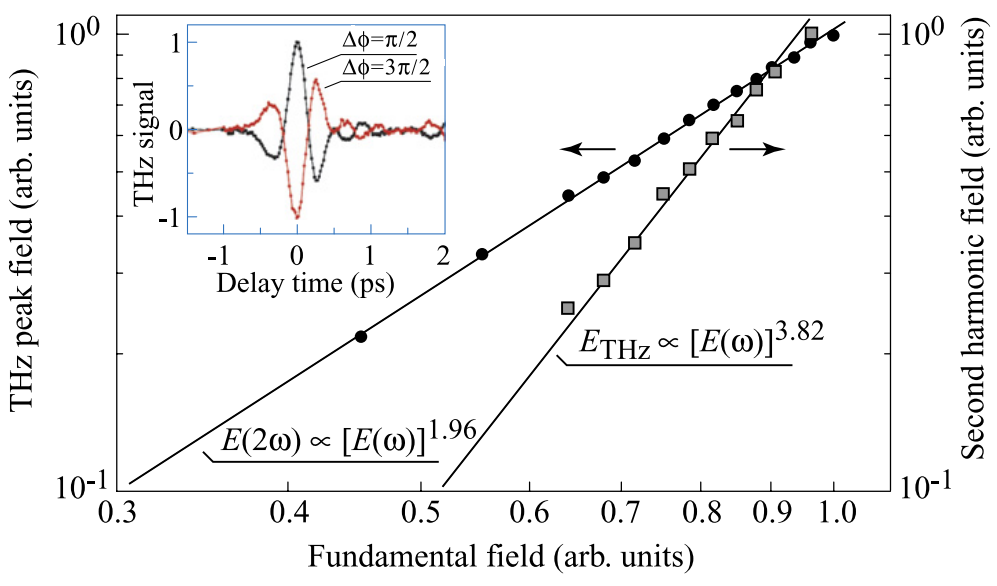

Fig. 23. Fundamental beam power dependence of the field $E(2 \omega)$ (black circles) and $\mathrm{THz}$ field proportional to the electric current generated in the sample (gray squares). Solid lines are corresponding power-law fits. Inset shows $\mathrm{THz}$ signal from the multilayer graphene sample as a function of time for two values of relative phases of the first and second harmonics $\Delta \phi$. The fundamental beam wavelength used in this experiment is $4.8 \mu \mathrm{m}$. After [63].
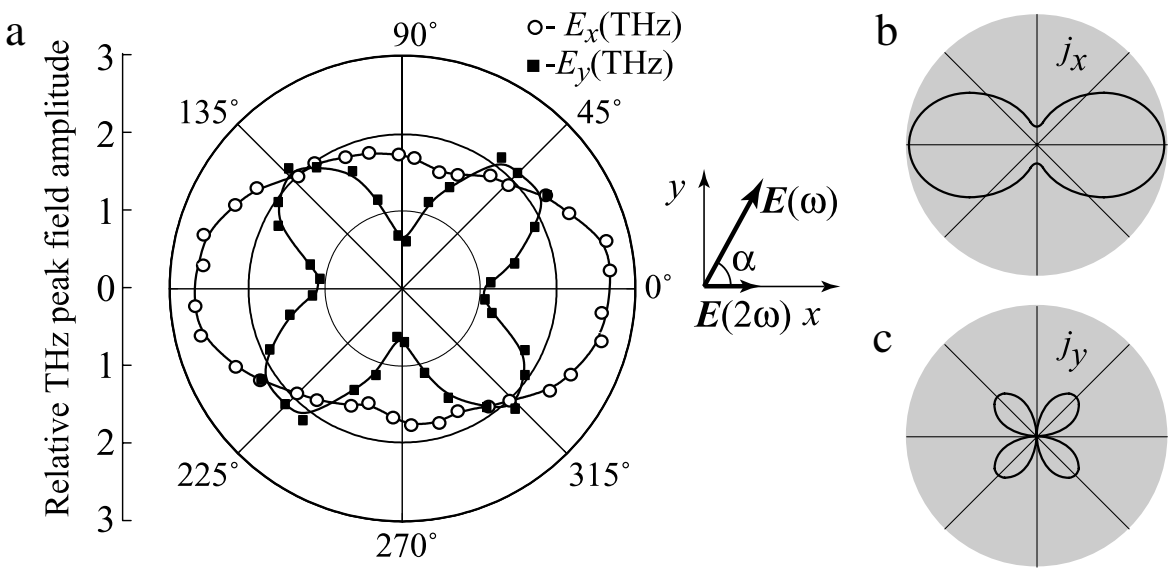

Fig. 24. (a) Experimentally measured $x$ and $y$ components of relative peak $\mathrm{THz}$ amplitude proportional to corresponding components of the photocurrent in the sample, Eq. (70), as a function of the polarization angle between $\omega$ and $2 \omega$ pulses. The field geometry is illustrated by the coordinate frame on the right, $\boldsymbol{E}(2 \omega) \| x$. Theoretical dependence of photocurrent $j_{x}$ [panel (b)] and $j_{y}$ components [panel (c)] calculated for a 70\% uncoupled-layer and 30\% coupled-layer. The boundary of the shaded circle represents unit amplitude. After [64].

dependence and are consistent with a third order optical process. Another proof of the third order optical process comes from the studies of polarization dependence of the relative THz peaks amplitudes carried out in Ref. [64]. This is shown in Fig. 24(a) where the dependence of the $\mathrm{THz}$ amplitudes, and, correspondingly, amplitudes of the photocurrent $\boldsymbol{j}$ on the angle between the polarization directions of $\omega$ and $2 \omega$ pulses, see Eq. (70). The data show that neither model of single layer graphene, nor that of a bilayer graphene describes experimental data. The results are in agreement with theoretical model [64] where the mixture of $70 \%$ of uncoupled layers and $30 \%$ of bilayers was assumed, demonstrating that the interlayer coupling modifies the polarization dependence of coherently controlled currents, as shown in Fig. 24(b), (c). This work demonstrates that (i) nonlinear electric transport can be studied on a femtosecond time scale and (ii) the photocurrents can be studied without necessarily to fabricate contacts to the graphene layer. Both advantages provide a unique access to dynamic of the nonlinear phenomena as well as allows one to characterize graphene layers in a contactless way.

\subsection{Summarizing remarks on the third and higher order effects in graphene}

A substantial number of possible third and higher order nonlinear effects has been observed during last three years in graphene and graphene-based structures. The effects include third and higher harmonic generation, frequency mixing, as well as coherent injection of ballistic photocurrents. Their experimental manifestations match well with available theoretical models, discussed above. The magnitude of third order effects, characterized by the third order susceptibility $\chi^{(3)}$, reaches $10^{-7}$ esu for graphene samples (see Section 6.3) for details. These values are in agreement with estimations, Section 6.2, and exceed by far $\chi^{(3)}$ reported for nonlinear crystals [174]. As for the coherent photogalvanic effect (injection of ballistic photocurrents), it was detected by a novel technique, based on THz emission induced by the transient current. This method reveals key features of the effect, namely, its nonlinear scaling with incident radiation amplitudes as well as the polarization dependence of the current. The functional behavior of the coherent photocurrents is well described by the present formalism, however, extraction of the effects magnitude is still a challenging task. 


\section{Conclusions and outlook}

The physics of nonlinear phenomena in graphene, although being young, has already resulted in a great variety of fascinating effects outlined here. Moreover, the field of nonlinear transport and optical phenomena in graphene opens new prospects for further studies. Many of the effects addressed so far are not yet fully understood and await novel experimental and theoretical approaches and detailed studies. Some of the theoretical predictions discussed here demand an experimental verification. The new horizons appear related with tailoring of the nonlinear response of the material by external magnetic field, strain or artificial combinations of graphene layers with other materials. Similar effects await to be studied in details in the systems with akin atomic arrangement or band structure, like Boron nitride (BN), Molybdenum disulfide $\left(\mathrm{MoS}_{2}\right)$ and various kinds of topological insulators, for which first results on high frequency nonlinear transport have already been published [175-178]. Finally, we anticipate, that such effects in graphene will soon find their applications both for material characterization and development of graphene-based nonlinear devices.

\section{Acknowledgments}

We are grateful to S.A. Mikhailov, V.V. Bel'kov, L.E. Golub, E.L. Ivchenko, V.A. Shalygin, and S.A. Tarasenko for valuable discussions.

This work was supported by DFG (SPP 1459 and GRK 1570), Linkage Grant of IB of BMBF at DLR, RFBR and RF President Grant NSh-5442.2012.2.

\section{References}

[1] K.S. Novoselov, A.K. Geim, S.V. Morozov, D. Jiang, Y. Zhang, S.V. Dubonos, I.V. Grigorieva, A.A. Firsov, Electric field effect in atomically thin carbon films, Science 306 (2004) 666.

[2] K.S. Novoselov, A.K. Geim, S.V. Morozov, D. Jiang, M.I. Katsnelson, I.V. Grigorieva, S.V. Dubonos, A.A. Firsov, Two-dimensional gas of massless Dirac fermions in graphene, Nature 438 (2005) 197.

[3] Y. Zhang, Y.-W. Tan, H.L. Stormer, P. Kim, Experimental observation of the quantum Hall effect and Berry's phase in graphene, Nature 438 (2005) 201.

[4] A.K. Geim, K.S. Novoselov, The rise of graphene, Nature Mater. 6 (2007) 183.

[5] A.H. Castro Neto, F. Guinea, N.M.R. Peres, K.S. Novoselov, A.K. Geim, The electronic properties of graphene, Rev. Modern Phys. 81 (2009) 109.

[6] P.R. Wallace, The band theory of graphite, Phys. Rev. 71 (1947) 622.

[7] J.W. McClure, Diamagnetism of graphite, Phys. Rev. 104 (1956) 666.

[8] J.C. Slonczewski, P.R. Weiss, Band structure of graphite, Phys. Rev. 109 (1958) 272.

[9] K.S. Novoselov, Z. Jiang, Y. Zhang, S.V. Morozov, H.L. Stormer, U. Zeitler, J.C. Maan, G.S. Boebinger, P. Kim, A.K. Geim, Room-temperature quantum Hall effect in graphene, Science 315 (2007) 1379.

[10] E. McCann, K. Kechedzhi, V.I. Fal'ko, H. Suzuura, T. Ando, B.L. Altshuler, Weak-localization magnetoresistance and valley symmetry in graphene, Phys. Rev. Lett. 97 (2006) 146805.

[11] F.V. Tikhonenko, D.W. Horsell, R.V. Gorbachev, A.K. Savchenko, Weak localization in graphene flakes, Phys. Rev. Lett. 100 (2008) 056802.

[12] M.I. Katsnelson, Zitterbewegung, chirality, and minimal conductivity in graphene, Eur. Phys. J. B 51 (2006) 157.

[13] K. Nomura, A.H. MacDonald, Quantum transport of massless Dirac fermions, Phys. Rev. Lett. 98 (2007) 076602.

[14] Y.-W. Tan, Y. Zhang, K. Bolotin, Y. Zhao, S. Adam, E.H. Hwang, S. Das Sarma, H.L. Stormer, P. Kim, Measurement of scattering rate and minimum conductivity in graphene, Phys. Rev. Lett. 99 (2007) 246803.

[15] N. Stander, B. Huard, D. Goldhaber-Gordon, Evidence for Klein tunneling in graphene p-n junctions, Phys. Rev. Lett. 102 (2009) 026807.

[16] A.F. Young, P. Kim, Quantum interference and carrier collimation in graphene heterojunctions, Nat. Phys. 5 (2009) 222.

[17] E. McCann, M. Koshino, The electronic properties of bilayer graphene (review), 2012. Arxiv e-prints: arXiv:1205.6953.

[18] G.W. Semenoff, Condensed-matter simulation of a three-dimensional anomaly, Phys. Rev. Lett. 53 (1984) 2449.

[19] F.D.M. Haldane, Model for a quantum Hall effect without Landau levels: condensed-matter realization of the parity anomaly, Phys. Rev. Lett. 61 (1988) 2015.

[20] S.V. Morozov, K.S. Novoselov, A.K. Geim, Electronic transport in graphene, Phys.-Usp. 51 (2008) 744

[21] Y.E. Lozovik, S.P. Merkulova, A.A. Sokolik, Collective electron phenomena in graphene, Phys.-Usp. 51 (2008) 727.

[22] A. Rycerz, J. Tworzydlo, C.W.J. Beenakker, Valley filter and valley valve in graphene, Nat. Phys. 3 (2007) 172.

[23] L.A. Falkovsky, Optical properties of graphene and IV-VI semiconductors, Phys.-Usp. 51 (2008) 887.

[24] N.M.R. Peres, Colloquium: the transport properties of graphene: an introduction, Rev. Modern Phys. 82 (2010) 2673.

[25] S. Das Sarma, S. Adam, E.H. Hwang, E. Rossi, Electronic transport in two-dimensional graphene, Rev. Modern Phys. 83 (2011) 407.

[26] F. Bonaccorso, Z. Sun, T. Hasan, A.C. Ferrari, Graphene photonics and optoelectronics, Nature Photonics 4 (2010) 611.

[27] N. Blombergen, Nonlinear Optics, Benjamin, New York, 1965.

[28] R.W. Boyd, Nonlinear Optics, Academic Press, San Diego, 1993

[29] A. Yariv, P. Yeh, Optical Waves in Crystals: Propagation and Control of Laser Radiation, J. Wiley \& Sons, New York, 2003.

[30] M. Wegener, Extreme Nonlinear Optics: An Introduction, Springer, Berlin, 2005.

[31] Y.R. Shen, The Principles of Nonlinear Optics, John Wiley \& Sons, New York, 2003.

[32] B. Sturman, V. Fridkin, The Photovoltaic and Photorefractive Effects in Non-Centrosymmetric Materials, Gordon \& Breach, Philadelphia, 1992.

[33] S. Ganichev, W. Prettl, Intense Terahertz Excitation of Semiconductors, Oxford University Press, 2006.

[34] E.L. Ivchenko, G.E. Pikus, Superlattices and Other Heterostructures, Springer, 1997.

[35] E.L. Ivchenko, Optical Spectroscopy of Semiconductor Nanostructures, Alpha Science, Harrow, UK, 2005.

[36] E.L. Ivchenko, B. Spivak, Chirality effects in carbon nanotubes, Phys. Rev. B 66 (2002) 155404.

[37] A.N. Obraztsov, D.A. Lyashenko, S. Fang, R.H. Baughman, P.A. Obraztsov, S.V. Garnov, Y.P. Svirko, Photon drag effect in carbon nanotube yarns, Appl. Phys. Lett. 94 (2009) 231112.

[38] V. Margulis, T. Sizikova, Theoretical study of third-order nonlinear optical response of semiconductor carbon nanotubes, Physica B 245 (1998) 173.

[39] G.Y. Slepyan, S.A. Maksimenko, V.P. Kalosha, A.V. Gusakov, J. Herrmann, High-order harmonic generation by conduction electrons in carbon nanotube ropes, Phys. Rev. A 63 (2001) 053808.

[40] E.J. Mele, P. Král, D. Tománek, Coherent control of photocurrents in graphene and carbon nanotubes, Phys. Rev. B 61 (2000) 7669.

[41] P. Král, E.J. Mele, D. Tománek, Photogalvanic effects in heteropolar nanotubes, Phys. Rev. Lett. 85 (2000) 1512.

[42] G.M. Mikheev, R.G. Zonov, A.N. Obraztsov, Y.P. Svirko, Giant optical rectification effect in nanocarbon films, Appl. Phys. Lett. 84 (2004) 4854. 
[43] G.M. Mikheev, A.G. Nasibulin, R.G. Zonov, A. Kaskela, E.I. Kauppinen, Photon-drag effect in single-walled carbon nanotube films, Nano Lett. 12 (2012) 77.

[44] A. Jorio, G. Dresselhaus, M.S. Dresselhaus (Eds.), Advanced Topics in the Synthesis, Structure, Properties and Applications, Springer, 2008.

[45] Z. Liu, X. Zhang, X. Yan, Y. Chen, J. Tian, Nonlinear optical properties of graphene-based materials, Chin. Sci. Bull. 57 (2012) 2971.

[46] Wang Jun, Chen Yu, Li Rihong, Dong Hongxing, Zhang Long, Lotya Mustafa, N. Coleman Jonathan, J. Blau Werner, Nonlinear optical properties of graphene and carbon nanotube composites, in: Siva Yellampalli (Ed.), Carbon Nanotubes - Synthesis, Characterization, Applications, 2011.

[47] S.A. Mikhailov, Nonlinear electromagnetic response of graphene, EPL 79 (2007) 27002.

[48] M. Dragoman, D. Neculoiu, G. Deligeorgis, G. Konstantinidis, D. Dragoman, A. Cismaru, A.A. Muller, R. Plana, Millimeter-wave generation via frequency multiplication in graphene, Appl. Phys. Lett. 97 (2010) 093101.

[49] J.J. Dean, H.M. van Driel, Second harmonic generation from graphene and graphitic films, Appl. Phys. Lett. 95 (2009) 261910.

[50] J.J. Dean, H.M. van Driel, Graphene and few-layer graphite probed by second-harmonic generation: theory and experiment, Phys. Rev. B 82 (2010) 125411.

[51] A.Y. Bykov, T.V. Murzina, M.G. Rybin, E.D. Obraztsova, Second harmonic generation in multilayer graphene induced by direct electric current, Phys. Rev. B 85 (2012) 121413.

[52] G. Hotopan, S. Ver Hoeye, C. Vazquez, R. Camblor, M. Fernández, F. Las Heras, P. Álvarez, R. Menéndez, Millimeter wave microstrip mixer based on graphene, Prog. Electromagn. Res. 118 (2011) 57.

[53] S.-Y. Hong, J.I. Dadap, N. Petrone, P.-C. Yeh, J. Hone, R.M. Osgood Jr., Optical third-harmonic generation in graphene, 2013. ArXiv e-prints: arXiv:1301.1697.

[54] N. Kumar, J. Kumar, C. Gerstenkorn, R. Wang, H.-Y. Chiu, A.L. Smirl, H. Zhao, Third harmonic generation in graphene and few-layer graphite films, 2013. ArXiv e-prints: arXiv: 1301.1042.

[55] E. Hendry, P.J. Hale, J.J. Moger, A.K. Savchenko, S.A. Mikhailov, Coherent nonlinear optical response of graphene, Phys. Rev. Lett. 105 (2010) 097401.

[56] N.L. Rangel, A. Gimenez, A. Sinitskii, J.M. Seminario, Graphene signal mixer for sensing applications, J. Phys. Chem. C 115 (2011) 12128.

[57] T. Gu, N. Petrone, J.F. McMillan, A. van der Zande, M. Yu, G.Q. Lo, D.L. Kwong, J. Hone, C.W. Wong, Regenerative oscillation and four-wave mixing in graphene optoelectronics, Nature Photonics 6 (2012) 554.

[58] J. Karch, P. Olbrich, M. Schmalzbauer, C. Zoth, C. Brinsteiner, M. Fehrenbacher, U. Wurstbauer, M.M. Glazov, S.A. Tarasenko, E.L. Ivchenko, D. Weiss, J. Eroms, R. Yakimova, S. Lara-Avila, S. Kubatkin, S.D. Ganichev, Dynamic Hall effect driven by circularly polarized light in a graphene layer, Phys. Rev. Lett. 105 (2010) 227402.

[59] J. Karch, P. Olbrich, M. Schmalzbauer, C. Brinsteiner, U. Wurstbauer, M.M. Glazov, S.A. Tarasenko, E.L. Ivchenko, D. Weiss, J. Eroms, S.D. Ganichev, Photon helicity driven electric currents in graphene, 2010. ArXiv e-prints: arXiv:1002.1047.

[60] M.V. Entin, L.I. Magarill, D.L. Shepelyansky, Theory of resonant photon drag in monolayer graphene, Phys. Rev. B 81 (2010) 165441.

[61] J. Karch, C. Drexler, P. Olbrich, M. Fehrenbacher, M. Hirmer, M.M. Glazov, S.A. Tarasenko, E.L. Ivchenko, B. Birkner, J. Eroms, D. Weiss, R. Yakimova, S. Lara-Avila, S. Kubatkin, M. Ostler, T. Seyller, S.D. Ganichev, Terahertz radiation driven chiral edge currents in graphene, Phys. Rev. Lett. 107 (2011)

[62] C. Jiang, V.A. Shalygin, V.Y. Panevin, S.N. Danilov, M.M. Glazov, R. Yakimova, S. Lara-Avila, S. Kubatkin, S.D. Ganichev, Helicity-dependent photocurrents in graphene layers excited by midinfrared radiation of a $\mathrm{CO}_{2}$ laser, Phys. Rev. B 84 (2011) 125429.

[63] D. Sun, C. Divin, J. Rioux, J.E. Sipe, C. Berger, W.A. de Heer, P.N. First, T.B. Norris, Coherent control of ballistic photocurrents in multilayer epitaxial graphene using quantum interference, Nano Lett. 10 (2010) 1293.

[64] D. Sun, J. Rioux, J.E. Sipe, Y. Zou, M.T. Mihnev, C. Berger, W.A. de Heer, P.N. First, T.B. Norris, Evidence for interlayer electronic coupling in multilayer epitaxial graphene from polarization-dependent coherently controlled photocurrent generation, Phys. Rev. B 85 (2012) 165427.

[65] D. Sun, C. Divin, M. Mihnev, T. Winzer, E. Malic, A. Knorr, J.E. Sipe, C. Berger, W.A. de Heer, P.N. First, T.B. Norris, Current relaxation due to hot carrier scattering in graphene, New J. Phys. 14 (2012) 105012.

[66] L. Prechtel, L. Song, D. Schuh, P. Ajayan, W. Wegscheider, A.W. Holleitner, Time-resolved ultrafast photocurrents and terahertz generation in freely suspended graphene, Nature Commun. 3 (2012) 01.

[67] M.W. Graham, S.-F. Shi, D.C. Ralph, J. Park, P.L. McEuen, Photocurrent measurements of supercollision cooling in graphene, Nat. Phys. 9 (2013) 103.

[68] S. Mai, S.V. Syzranov, K.B. Efetov, Photocurrent in a visible-light graphene photodiode, Phys. Rev. B 83 (2011) 033402.

[69] F. Xia, T. Mueller, R. Golizadeh-Mojarad, M. Freitag, Y.-m. Lin, J. Tsang, V. Perebeinos, P. Avouris, Photocurrent imaging and efficient photon detection in a graphene transistor, Nano Lett. 9 (2009) 1039.

[70] S.V. Syzranov, M.V. Fistul, K.B. Efetov, Effect of radiation on transport in graphene, Phys. Rev. B 78 (2008) 045407.

[71] R. Wu, Y. Zhang, S. Yan, F. Bian, W. Wang, X. Bai, X. Lu, J. Zhao, E. Wang, Purely coherent nonlinear optical response in solution dispersions of graphene sheets, Nano Lett. 11 (2011) 5159.

[72] S. Chu, S. Wang, Q. Gong, Ultrafast third-order nonlinear optical properties of graphene in aqueous solution and polyvinyl alcohol film, Chem. Phys. Lett. 523 (2012) 104.

[73] Xiao-Qing Yan, Zhi-Bo Liu, Jun Yao, Xin Zhao, Xu-Dong Chen, Fei Xing, Yongsheng Chen, Jian-Guo Tian, Experimental observation of polarizationdependent ultrafast carrier dynamics in multi-layer graphene, 2013. Arxiv e-prints: arXiv:1301.1743.

[74] S.A. Mikhailov, Theory of the giant plasmon-enhanced second-harmonic generation in graphene and semiconductor two-dimensional electron systems, Phys. Rev. B 84 (2011) 045432.

[75] T.J. Echtermeyer, L. Britnell, P.K. Jasnos, A. Lombardo, R.V. Gorbachev, A.N. Grigorenko, A.K. Geim, A.C. Ferrari, K.S. Novoselov, Strong plasmonic enhancement of photovoltage in graphene, Nature Commun. 2 (2011) 458.

[76] A.N. Grigorenko, M. Polini, K.S. Novoselov, Graphene plasmonics, Nature Photonics 6 (2012) 749.

[77] F.J. López-Rodríguez, G.G. Naumis, Analytic solution for electrons and holes in graphene under electromagnetic waves: gap appearance and nonlinear effects, Phys. Rev. B 78 (2008) 201406(R).

[78] F. Bassani, G. Pastori-Parravicini, Electronic States and Optical Transitions in Solids, Pergamon Press, Oxford, New York, 1975.

[79] A. Zunger, Self-consistent LCAO calculation of the electronic properties of graphite. I. The regular graphite lattice, Phys. Rev. B 17 (1978) 626.

[80] B.E.A. Saleh, M.C. Teich, Fundamentals of Photonics, John Wiley \& Sons, New York, 2003.

[81] M. Born, E. Wolf, Principles of Optics: Electromagnetic Theory of Propagation, Interference and Diffraction of Light, Cambridge University Press, 1999.

[82] S.D. Ganichev, E.L. Ivchenko, W. Prettl, Photogalvanic effects in quantum wells, Physica E 14 (2002) 166.

[83] S.D. Ganichev, W. Prettl, Spin photocurrents in quantum wells, J. Phys.: Condens. Matter 15 (2003) R935.

[84] E. Ivchenko, S. Ganichev, in: M. Dyakonov (Ed.), Spin Physics in Semiconductors, Springer, 2008.

[85] I.D. Yaroshetskii, S.M. Ryvkin, Semiconductor Physics, Cons. Bureau, New York, 1986.

[86] A.F. Gibson, M.F. Kimmitt, Infrared and Millimeter Waves, Vol. 3, Detection of Radiation, Academic Press, New York, 1980, pp. 181-217.

[87] H.M. Barlow, Application of the Hall effect in a semi-conductor to the measurement of power in an electromagnetic field, Nature 173 (1954) 41.

[88] E.L. Ivchenko, G.E. Pikus, Semiconductor Physics, Cons. Bureau, New York, 1986.

[89] V.I. Belinicher, On the mechanisms underlying the circular drag effect, Sov. Phys.-Solid State 23 (1981) 2012

[90] V. Shalygin, H. Diehl, C. Hoffmann, S. Danilov, T. Herrle, S. Tarasenko, D. Schuh, C. Gerl, W. Wegscheider, W. Prettl, S. Ganichev, Spin photocurrents and the circular photon drag effect in (110)-grown quantum well structures, JETP Lett. 84 (2007) 570.

[91] T. Hatano, T. Ishihara, S.G. Tikhodeev, N.A. Gippius, Transverse photovoltage induced by circularly polarized light, Phys. Rev. Lett. 103 (2009) 103906.

[92] W. Weber, L.E. Golub, S.N. Danilov, J. Karch, C. Reitmaier, B. Wittmann, V.V. Bel'kov, E.L. Ivchenko, Z.D. Kvon, N.Q. Vinh, A.F.G. van der Meer, B. Murdin, S.D. Ganichev, Quantum ratchet effects induced by terahertz radiation in GaN-based two-dimensional structures, Phys. Rev. B 77 (2008) 245304

[93] Y.Y. Kiselev, L.E. Golub, Optical and photogalvanic properties of graphene superlattices formed by periodic strain, Phys. Rev. B 84 (2011) 235440.

[94] A.V. Nalitov, L.E. Golub, E.L. Ivchenko, Ratchet effects in two-dimensional systems with a lateral periodic potential, Phys. Rev. B 86 (2012) 115301.

[95] T. Ohta, A. Bostwick, T. Seyller, K. Horn, E. Rotenberg, Controlling the electronic structure of bilayer graphene, Science 313 (2006) 951. 
[96] E.V. Castro, K.S. Novoselov, S.V. Morozov, N.M.R. Peres, J.M.B.L. dos Santos, J. Nilsson, F. Guinea, A.K. Geim, A.H.C. Neto, Biased bilayer graphene: semiconductor with a gap tunable by the electric field effect, Phys. Rev. Lett. 99 (2007) 216802.

[97] F. Wang, Y. Zhang, C. Tian, C. Girit, A. Zettl, M. Crommie, Y.R. Shen, Gate-variable optical transitions in graphene, Science 320 (2008) 206.

[98] A.S. Mayorov, D.C. Elias, M. Mucha-Kruczynski, R.V. Gorbachev, T. Tudorovskiy, A. Zhukov, S.V. Morozov, M.I. Katsnelson, V.I. Fal'ko, A.K. Geim, K.S. Novoselov, Interaction-driven spectrum reconstruction in bilayer graphene, Science 333 (2011) 860

[99] W. Bao, L. Jing, J. Velasco, Y. Lee, G. Liu, D. Tran, B. Standley, M. Aykol, S.B. Cronin, D. Smirnov, M. Koshino, E. McCann, M. Bockrath, C.N. Lau, Stackingdependent band gap and quantum transport in trilayer graphene, Nat. Phys. 7 (2011) 948.

[100] L. Zhang, Y. Zhang, J. Camacho, M. Khodas, I. Zaliznyak, The experimental observation of quantum Hall effect of $l=3$ chiral quasiparticles in trilayer graphene, Nat. Phys. 7 (2011) 953.

[101] C.H. Lui, Z. Li, K.F. Mak, E. Cappelluti, T.F. Heinz, Observation of an electrically tunable band gap in trilayer graphene, Nat. Phys. 7 (2011) 944.

[102] J.L. Mañes, F. Guinea, M.A.H. Vozmediano, Existence and topological stability of Fermi points in multilayered graphene, Phys. Rev. B 75 (2007) 155424.

[103] L.M. Malard, M.H.D. Guimarães, D.L. Mafra, M.S.C. Mazzoni, A. Jorio, Group-theory analysis of electrons and phonons in N-layer graphene systems, Phys. Rev. B 79 (2009) 125426.

[104] L.E. Golub, S.A. Tarasenko, M.V. Entin, L.I. Magarill, Valley separation in graphene by polarized light, Phys. Rev. B 84 (2011) 195408.

[105] R.R. Hartmann, M.E. Portnoi, Optoelectronic Properties of Carbon-based Nanostructures: Steering Electrons in Graphene by Electromagnetic Fields, LAP LAMBERT Academic Publishing, Saarbrucken, 2011.

[106] M. Glazov, Second harmonic generation in graphene, JETP Lett. 93 (2011) 366.

[107] M. Bass, P.A. Franken, J.F. Ward, Optical rectification, Phys. Rev. 138 (1965) A534.

[108] D. Côté, N. Laman, H.M. van Driel, Rectification and shift currents in GaAs, Appl. Phys. Lett. 80 (2002) 905.

[109] L.E. Gurevich, A.A. Rumyantsev, Theory of the photoelectric effect in finite crystals at high frequencies and in the presence of an external magnetic field, Sov. Phys.-Solid State 9 (1967) 55.

[110] V.I. Perel', Ya.M. Pinskii, Constant current in conducting media due to a high-frequency electron electromagnetic field, Sov. Phys.-Solid State 15 (1973) 688 .

[111] H.W.K. Tom, T.F. Heinz, Y.R. Shen, Second-harmonic reflection from silicon surfaces and its relation to structural symmetry, Phys. Rev. Lett. 51 (1983) $1983-1986$.

[112] R.R. Nair, P. Blake, A.N. Grigorenko, K.S. Novoselov, T.J. Booth, T. Stauber, N.M.R. Peres, A.K. Geim, Fine structure constant defines visual transparency of graphene, Science 320 (2008) 1308.

[113] A.A. Grinberg, S. Luryi, Theory of the photon-drag effect in a two-dimensional electron gas, Phys. Rev. B 38 (1988) 87.

[114] C. Drexler, S.A. Tarasenko, P. Olbrich, J. Karch, M. Hirmer, F. Muller, M. Gmitra, J. Fabian, R. Yakimova, S. Lara-Avila, S. Kubatkin, M. Wang, R. Vajtai, P.M. Ajayan, J. Kono, S.D. Ganichev, Magnetic quantum ratchet effect in graphene, Nature Nanotechnol. 8 (2013) 104.

[115] S. Lara-Avila, K. Moth-Poulsen, R. Yakimova, T. Bjornholm, V. Fal'ko, A. Tzalenchuk, S. Kubatkin, Non-volatile photochemical gating of an epitaxial graphene, Adv. Mater. 23 (2011) 878.

[116] A. Tzalenchuk, S. Lara-Avila, A. Kalaboukhov, S. Paolillo, M. Syvajarvi, R. Yakimova, O. Kazakova, T.J.B.M. Janssen, V. Fal'ko, S. Kubatkin, Towards a quantum resistance standard based on epitaxial graphene, Nature Nanotechnol. 5 (2010) 186.

[117] K.V. Emtsev, A. Bostwick, K. Horn, J. Jobst, G.L. Kellogg, L. Ley, J.L. McChesney, T. Ohta, S.A. Reshanov, J. Rohrl, E. Rotenberg, A.K. Schmid, D. Waldmann, H.B. Weber, T. Seyller, Towards wafer-size graphene layers by atmospheric pressure graphitization of silicon carbide, Nature Mater. 8 (2009) 203.

[118] S.D. Ganichev, J. Diener, I.N. Yassievich, W. Prettl, B.K. Meyer, K.W. Benz, Tunnelling ionization of autolocalized DX-centers in terahertz fields, Phys. Rev. Lett. 75 (1995) 1590

[119] S.D. Ganichev, I.N. Yassievich, W. Prettl, Tunneling ionization of deep centers in terahertz electric fields, topical review, J. Phys.: Condens. Matter 14 (2002) R1263.

[120] S.D. Ganichev, S.A. Tarasenko, V.V. Bel'kov, P. Olbrich, W. Eder, D.R. Yakovlev, V. Kolkovsky, W. Zaleszczyk, G. Karczewski, T. Wojtowicz, D. Weiss, Spin currents in diluted magnetic semiconductors, Phys. Rev. Lett. 102 (2009) 156602.

[121] Z.D. Kvon, S.N. Danilov, N.N. Mikhailov, S.A. Dvoretsky, S.D. Ganichev, Cyclotron resonance photoconductivity of a two-dimensional electron gas in HgTe quantum wells, Physica E 40 (2008) 1885.

[122] V.V. Bel'kov, S.D. Ganichev, Magneto-gyrotropic effects in semiconductor quantum wells, review, Semicond. Sci. Technol. 23 (2008) 114003.

[123] E. Ziemann, S.D. Ganichev, I.N. Yassievich, V.I. Perel, W. Prettl, Characterization of deep impurities in semiconductors by terahertz tunneling ionization, J. Appl. Phys. 87 (2000) 3843.

[124] J.C.W. Song, M.S. Rudner, C.M. Marcus, L.S. Levitov, Hot carrier transport and photocurrent response in graphene, Nano Lett. 11 (2011) 4688; A.A. Varlamov, A.V. Kavokin, I.A. Luk'yanchuk, S.G. Sharapov, Anomalous thermoelectric and thermomagnetic properties of graphene, Phys. Usp. 55 (2012) 1146;

S.V. Koniakhin, E.D. Eidelman, Phonon drag thermopower in graphene in equipartition regime, EPL 103 (2013) 37006

[125] S. Tarasenko, Orbital mechanism of the circular photogalvanic effect in quantum wells, JETP Lett. 85 (2007) 182.

[126] P. Olbrich, S.A. Tarasenko, C. Reitmaier, J. Karch, D. Plohmann, Z.D. Kvon, S.D. Ganichev, Observation of the orbital circular photogalvanic effect, Phys. Rev. B 79 (2009) 121302

[127] S.A. Tarasenko, Direct current driven by ac electric field in quantum wells, Phys. Rev. B 83 (2011) 035313.

[128] V.L. Gurevich, R. Laiho, Photomagnetism of metals: microscopic theory of the photoinduced surface current, Phys. Rev. B 48 (1993) 8307

[129] V. Gurevich, R. Laiho, Photomagnetism of metals. First observation of dependence on polarization of light, Phys. Solid State 42 (2000) 1807.

[130] L. Magarill, M. Entin, Surface photogalvanic effect in metals, JETP 54 (1981) 531.

[131] V.L. Al'perovich, V.I. Belinicher, V.N. Novikov, A.S. Terekhov, Surface photovoltaic effect in gallium arsenide, JETP Lett. 31 (1980) 546.

[132] K. Bolotin, K. Sikes, Z. Jiang, M. Klima, G. Fudenberg, J. Hone, P. Kim, H. Stormer, Ultrahigh electron mobility in suspended graphene, Solid State Commun. $146(2008) 351$.

[133] C. Casiraghi, A. Hartschuh, H. Qian, S. Piscanec, C. Georgi, A. Fasoli, K.S. Novoselov, D.M. Basko, A.C. Ferrari, Raman spectroscopy of graphene edges, Nano Lett. 9 (2009) 1433.

[134] S. Heydrich, M. Hirmer, C. Preis, T. Korn, J. Eroms, D. Weiss, C. Schueller, Scanning Raman spectroscopy of graphene antidot lattices: evidence for systematic p-type doping, Appl. Phys. Lett. 97 (2010) 043113.

[135] E.J.H. Lee, K. Balasubramanian, R.T. Weitz, M. Burghard, K. Kern, Contact and edge effects in graphene devices, Nature Nanotechnol. 3 (2008) 486.

[136] M. Ostler, F. Speck, M. Gick, T. Seyller, Automated preparation of high-quality epitaxial graphene on 6H-SiC(0001), Phys. Status Solidi b 247 (2010) 2924.

[137] W.S. Bao, S.Y. Liu, X.L. Lei, Hot-electron transport in graphene driven by intense terahertz fields, Phys. Lett. A 374 (2010) 1266.

[138] F.T. Vasko, V. Ryzhii, Photoconductivity of intrinsic graphene, Phys. Rev. B 77 (2008) 195433.

[139] M. Trushin, J. Schliemann, Anisotropic photoconductivity in graphene, EPL 96 (2011) 37006.

[140] N.M. Vildanov, Optical conductivity and electron-hole pair creation in graphene, J. Phys.: Condens. Matter 21 (2009) 445802.

[141] D. Sun, G. Aivazian, A.M. Jones, J.S. Ross, W. Yao, D. Cobden, X. Xu, Ultrafast hot-carrier-dominated photocurrent in graphene, Nature Nanotechnol. $7(2012) 114$.

[142] T. Oka, H. Aoki, Photovoltaic Hall effect in graphene, Phys. Rev. B 79 (2009) 081406.

[143] O.V. Kibis, Metal-insulator transition in graphene induced by circularly polarized photons, Phys. Rev. B 81 (2010) 165433.

[144] Z. Gu, H.A. Fertig, D.P. Arovas, A. Auerbach, Floquet spectrum and transport through an irradiated graphene ribbon, Phys. Rev. Lett. 107 (2011) 216601.

[145] T. Kitagawa, T. Oka, A. Brataas, L. Fu, E. Demler, Transport properties of nonequilibrium systems under the application of light: photoinduced quantum Hall insulators without Landau levels, Phys. Rev. B 84 (2011) 235108.

[146] Hernan L. Calvo, Horacio M. Pastawski, Stephan Roche, Luis E.F. Foa Torres, Tuning laser-induced band gaps in graphene, Appl. Phys. Lett. 98 (2011) 232103. 
[147] Hernan L. Calvo, Pablo M. Perez-Piskunow, Stephan Roche, Luis E.F. Foa Torres, Laser-induced effects on the electronic features of graphene nanoribbons, Appl. Phys. Lett. 101 (2012) 253506.

[148] Eric Suarez Morell, Luis E.F. Foa Torres, Radiation effects on the electronic properties of bilayer graphene, Phys. Rev. B 86 (2012) 125449.

[149] F.T. Vasko, Carrier heating and high-order harmonics generation in doped graphene by a strong ac electric field, 2010. ArXiv e-prints: arXiv:1011.4841.

[150] G.M. Shmelev, N.H. Shon, G.I. Tsurkan, Photostimulated even acousto-electric effect, Izv. Vyssh. Uchebn. Zaved. Fiz. 28 (1985) 84.

[151] M.V. Entin, Theory of coherent photogalvanic effect, Sov. Phys. Semicond. 23 (1989) 664.

[152] M.I. Dyakonov (Ed.), Spin Physics in Semiconductors, Springer-Verlag, Berlin, Heidelberg, 2008.

[153] S.A. Mikhailov, K. Ziegler, Nonlinear electromagnetic response of graphene: frequency multiplication and the self-consistent-field effects, J. Phys.: Condens. Matter 20 (2008) 384204.

[154] F. Bass, A. Tetervov, High-frequency phenomena in semiconductor superlattices, Phys. Rep. 140 (1986) 237.

[155] S. Wu, L. Mao, A.M. Jones, W. Yao, C. Zhang, X. Xu, Quantum-enhanced tunable second-order optical nonlinearity in bilayer graphene, Nano Lett. 12 (2012) 2032.

[156] K.N. Alekseev, M.V. Erementchouk, F.V. Kusmartsev, Direct-current generation due to wave mixing in semiconductors, EPL 47 (1999) 595.

[157] A. O'Hare, F.V. Kusmartsev, K.I. Kugel, A stable flat form of two-dimensional crystals: could graphene, silicene, germanene be minigap semiconductors? Nano Lett. 12 (2012) 1045.

[158] J. Rioux, G. Burkard, J.E. Sipe, Current injection by coherent one- and two-photon excitation in graphene and its bilayer, Phys. Rev. B 83 (2011) 195406.

[159] A.R. Wright, X.G. Xu, J.C. Cao, C. Zhang, Strong nonlinear optical response of graphene in the terahertz regime, Appl. Phys. Lett. 95 (2009) 072101.

[160] S. Shareef, Y.S. Ang, C. Zhang, Room-temperature strong terahertz photon mixing in graphene, J. Opt. Soc. Am. B 29 (2012) 274.

[161] X. Yao, A. Belyanin, Giant optical nonlinearity of graphene in a strong magnetic field, Phys. Rev. Lett. 108 (2012) 255503.

[162] R. Camblor, S.V. Hoeye, G. Hotopan, C. Vázquez, M. Fernández, F.L. Heras, P. Álvarez, R. Menéndez, Microwave frequency tripler based on a microstrip gap with graphene, J. Electromagn. Waves Appl. 25 (2011) 1921.

[163] M. Orlita, C. Faugeras, P. Plochocka, P. Neugebauer, G. Martinez, D.K. Maude, A.-L. Barra, M. Sprinkle, C. Berger, W.A. de Heer, M. Potemski, Approaching the Dirac point in high-mobility multilayer epitaxial graphene, Phys. Rev. Lett. 101 (2008) 267601.

[164] C. Faugeras, A. Nerriere, M. Potemski, A. Mahmood, E. Dujardin, C. Berger, W.A. de Heer, Few-layer graphene on SiC, pyrolitic graphite, and graphene: a Raman scattering study, Appl. Phys. Lett. 92 (2008) 011914.

[165] J. Hass, F. Varchon, J.E. Millan-Otoya, M. Sprinkle, N. Sharma, W.A. de Heer, C. Berger, P.N. First, L. Magaud, E.H. Conrad, Why multilayer graphene on $4 \mathrm{H}-\mathrm{SiC}(000 \overline{1})$ behaves like a single sheet of graphene, Phys. Rev. Lett. 100 (2008) 125504.

[166] D.L. Miller, K.D. Kubista, G.M. Rutter, M. Ruan, W.A. de Heer, P.N. First, J.A. Stroscio, Observing the quantization of zero mass carriers in graphene, Science 324 (2009) 924.

[167] M.L. Sadowski, G. Martinez, M. Potemski, C. Berger, W.A. De Heer, Landau level spectroscopy of ultrathin graphite layers, Phys. Rev. Lett. 97 (2006) 266405.

[168] X. Wu, X. Li, Z. Song, C. Berger, W.A De Heer, Weak antilocalization in epitaxial graphene: evidence for chiral electrons, Phys. Rev. Lett. 98 (2007) 136801.

[169] I.A. Luk'yanchuk, Y. Kopelevich, Phase analysis of quantum oscillations in graphite, Phys. Rev. Lett. 93 (2004) 166402.

[170] P.R. Smith, D.H. Auston, M.C. Nuss, Subpicosecond photoconductive dipole antennas, IEEE J. Quantum Electron. QE-24 (1988) 255.

[171] X.-C. Zhang, B.B. Hu, J.T. Darrow, D.H. Auston, Generation of femtosecond electromagnetic pulses from semiconductor surfaces, Appl. Phys. Lett. 56 (1990) 1011.

[172] Kiyomi Sakai, Terahertz Optoelectronics (Topics in Applied Physics), Springer, 2005

[173] Yun-Shik Lee, Principles of Terahertz Science and Technology, Springer, 2009.

[174] R.W. Boyd, G.L. Fischer, in: K.H. Jürgen Buschow, Robert W. Cahn, Merton C. Flemings, Bernard Ilschner, Edward J. Kramer, Subhash Mahajan, Patrick Veyssière (Eds.), Encyclopedia of Materials: Science and Technology, Elsevier, 2001, 2011.

[175] D. Hsieh, J.W. McIver, D.H. Torchinsky, D.R. Gardner, Y.S. Lee, N. Gedik, Nonlinear optical probe of tunable surface electrons on a topological insulator, Phys. Rev. Lett. 106 (2011) 057401.

[176] J.C.W. Song, L.S. Levitov, System-wide photocurrent response in gapless materials, 2011. ArXiv e-prints: arXiv:1112.5654

[177] P. Hosur, Circular photogalvanic effect on topological insulator surfaces: Berry-curvature-dependent response, Phys. Rev. B 83 (2011) 035309.

[178] Balázs Dóra, Jérôme Cayssol, Ferenc Simon, Roderich Moessner, Optically engineering the topological properties of a spin Hall insulator, Phys. Rev. Lett. 108 (2012) 056602. 